\title{
Regio- and Enantioselective Friedel-Crafts Benzhydrylation of Indoles in Carbocyclic Ring with ortho-Quinomethanes: Access to Chiral Diarylindolylmethanes
}

Ming-Ming Chu, Xue-Yang Chen, Yi-Feng Wang,* Suo-Suo Qi, Zhen-Hui Jiang, Dan-Qian Xu,* and Zhen-Yuan $\mathrm{Xu}$

State Key Laboratory Breeding Base of Green Chemistry-Synthesis Technology, Key Laboratory of Green Pesticides and Cleaner Production Technology of Zhejiang Province, Department of Green Chemistry and Technology, Zhejiang University of Technology, Hangzhou 310014, P. R. China. E-mail: chrc@,zjut.edu.cn

\section{List of Contents}

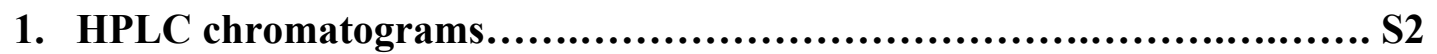

2. ${ }^{1} \mathrm{H}-\mathrm{NMR}$ and ${ }^{13} \mathrm{C}-\mathrm{NMR}$ spectra...........................................S29

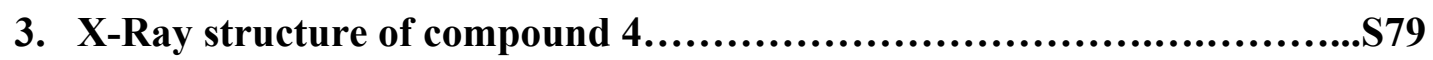




\section{HPLC chromatograms}<smiles>COc1cccc([C@H](c2ccccc2)c2ccc3[nH]ccc3c2O)c1O</smiles>
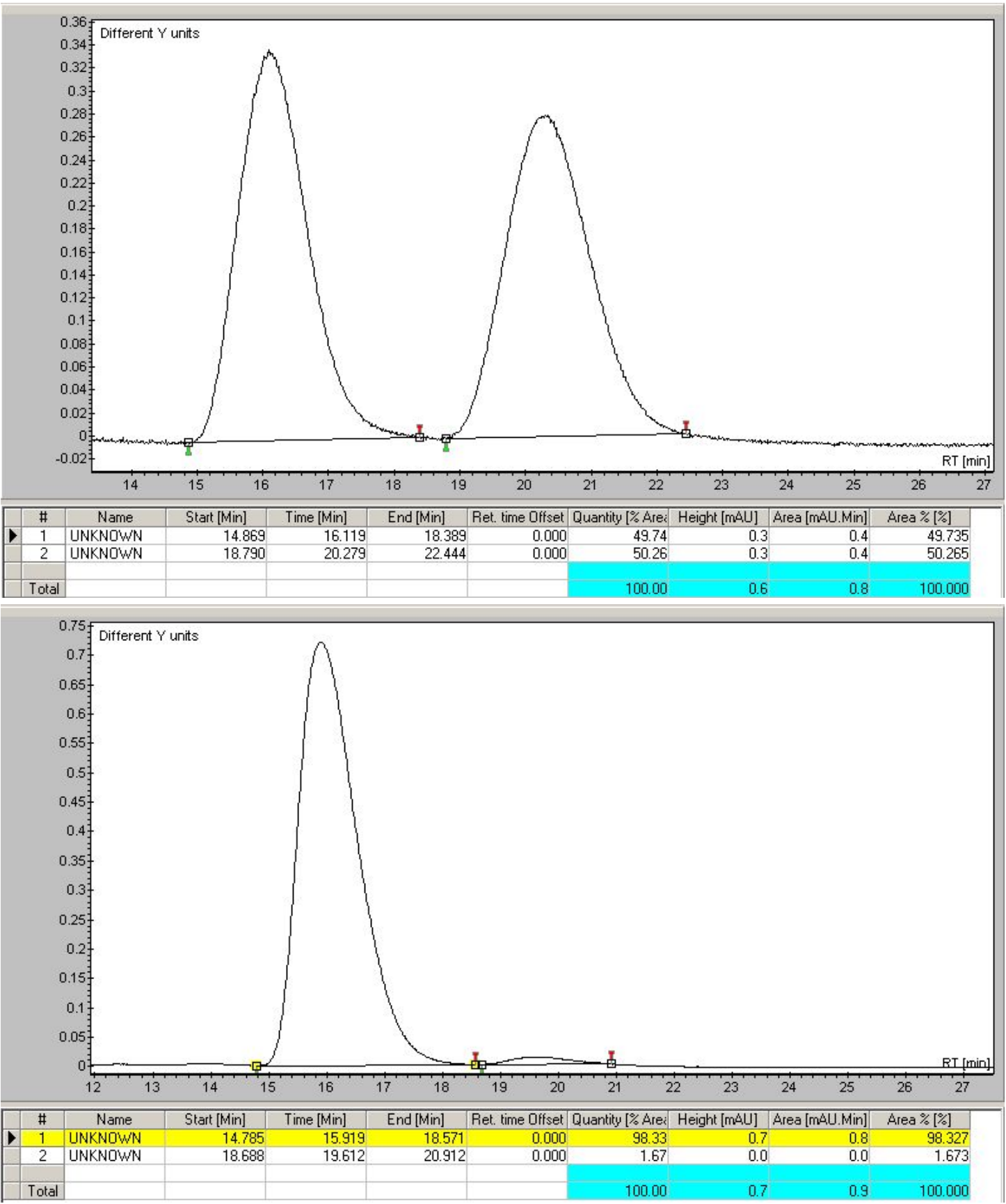


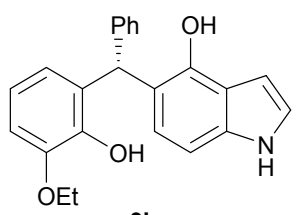

3 ba
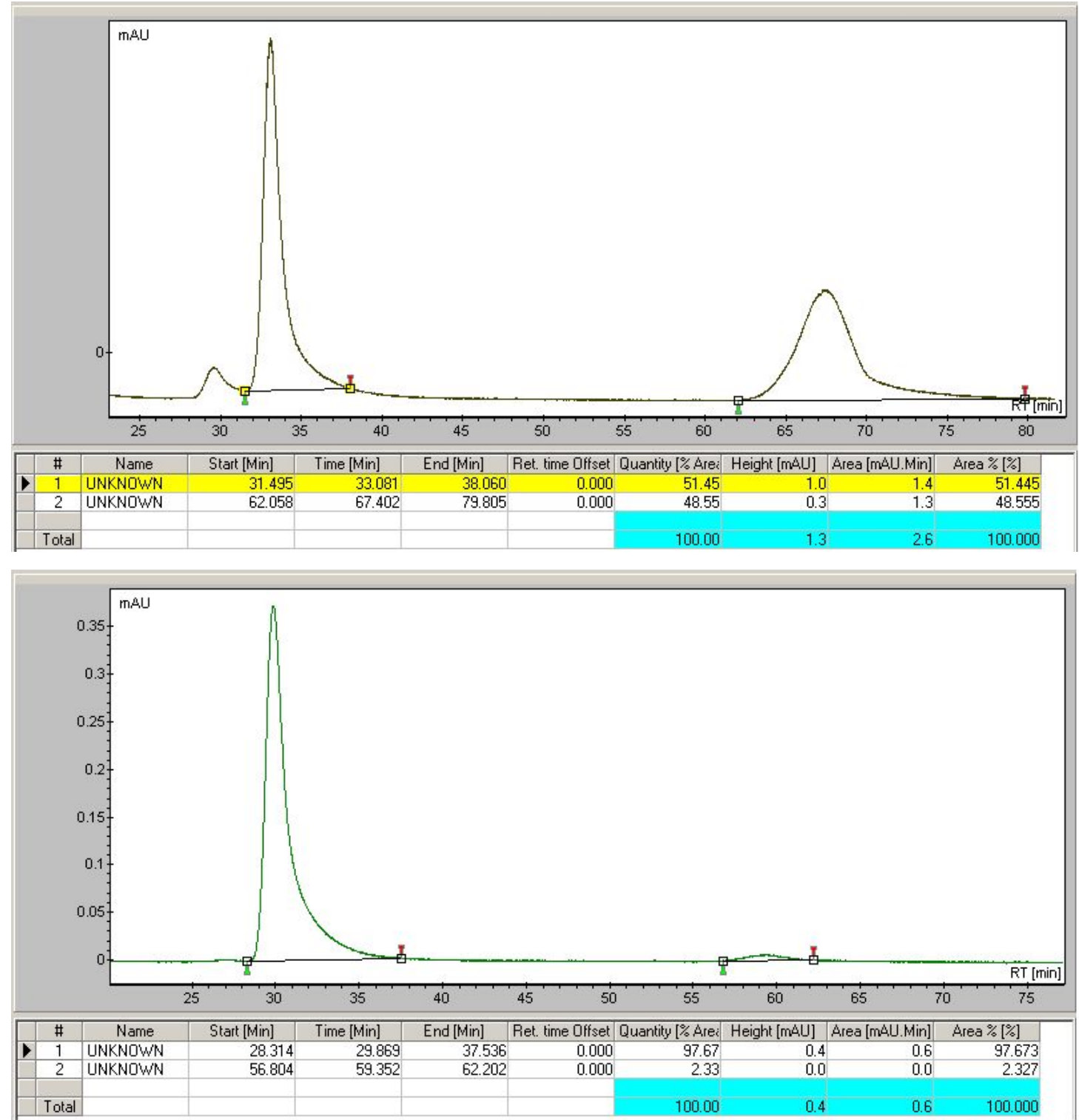
<smiles>COc1ccc(C(c2ccc3[nH]ccc3c2O)c2ccc3[nH]ccc3c2O)c(O)c1</smiles>
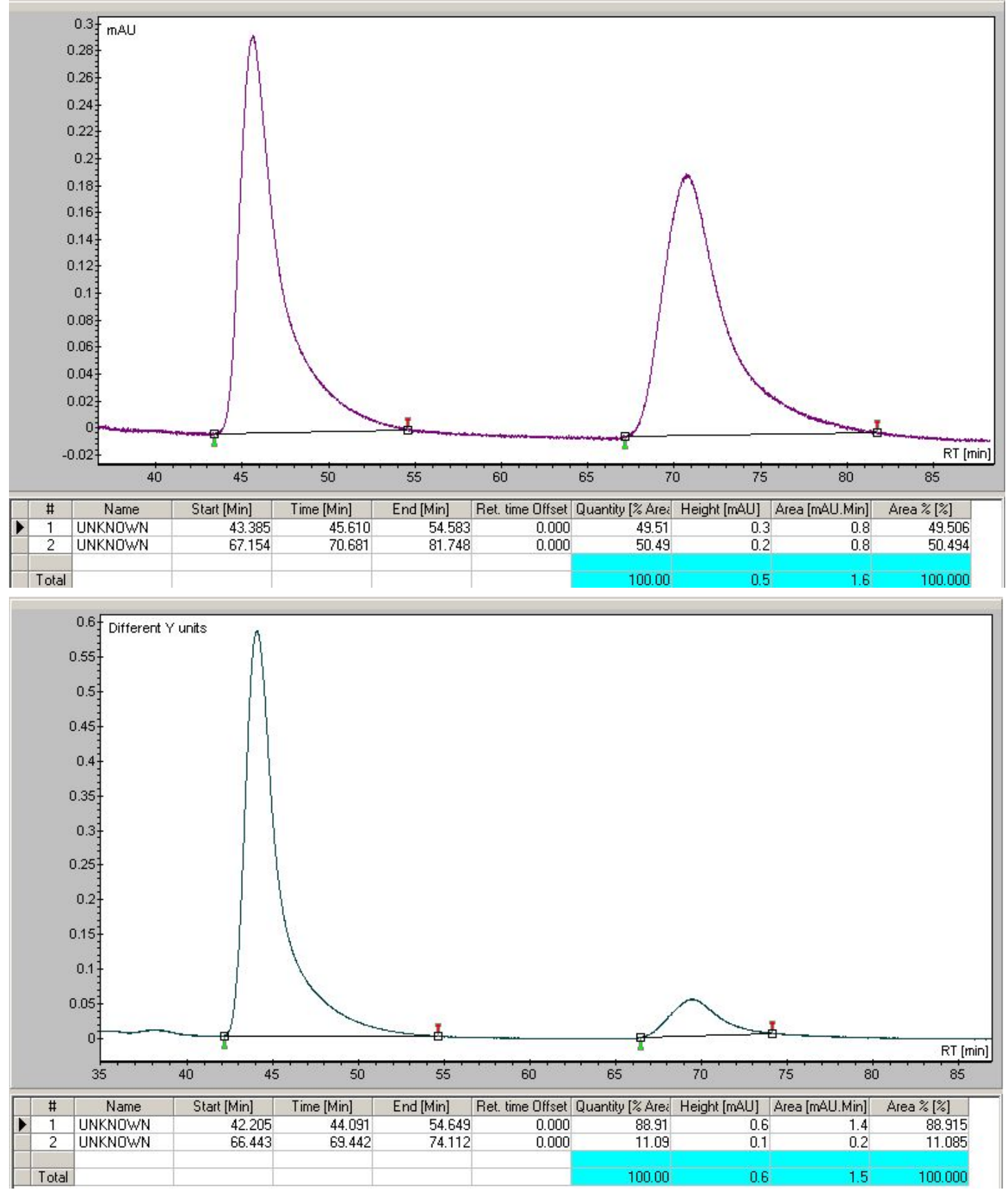
<smiles>COc1cc(Br)cc(C(c2ccccc2)c2ccc3[nH]ccc3c2O)c1O</smiles>

3da
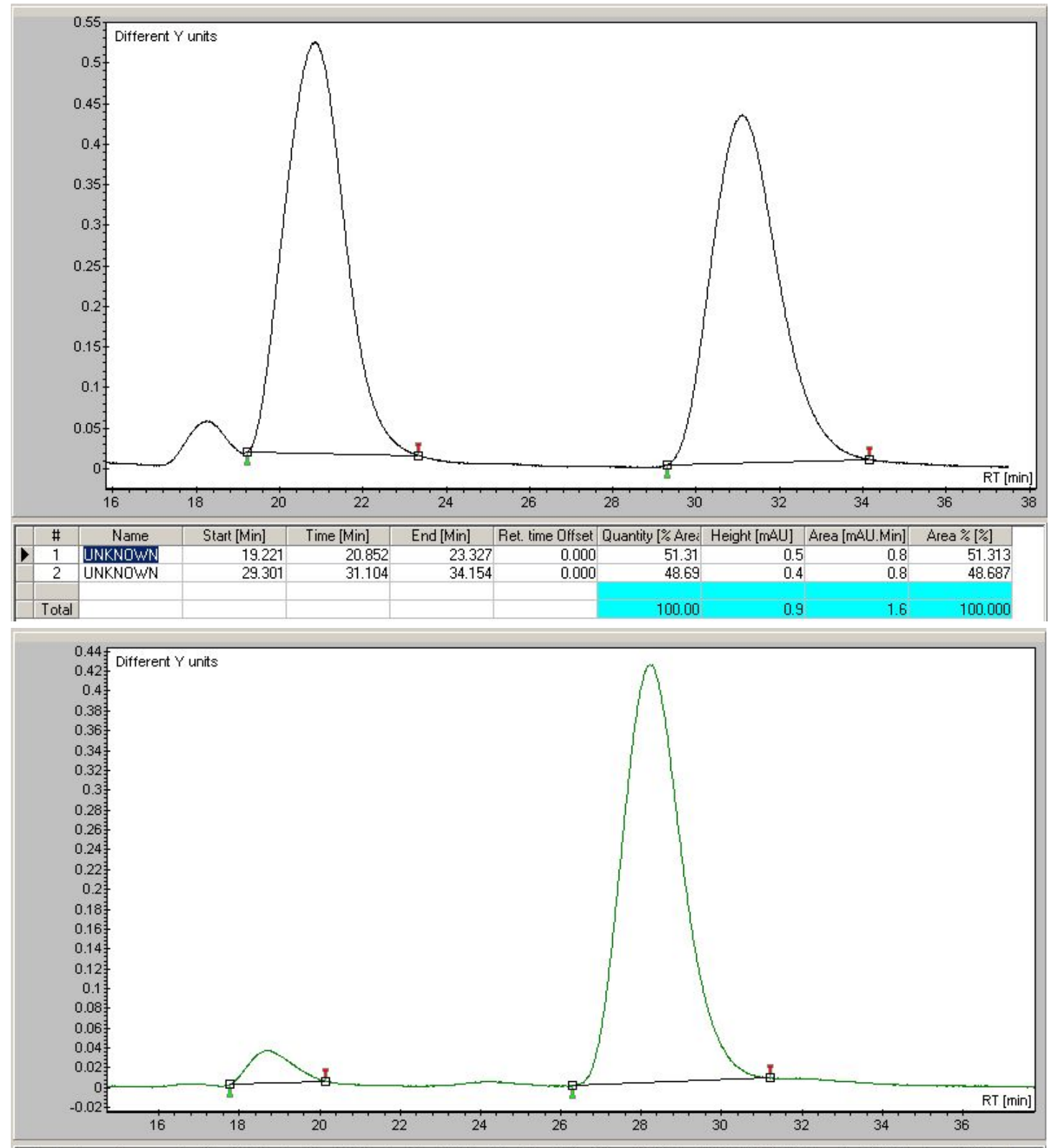

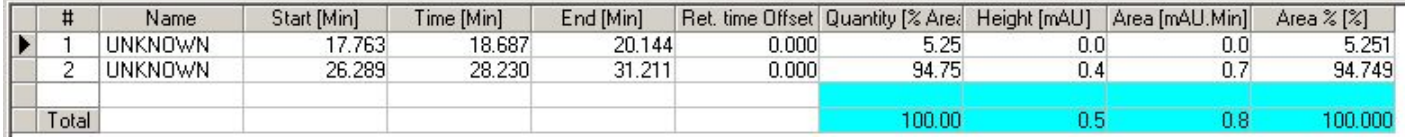



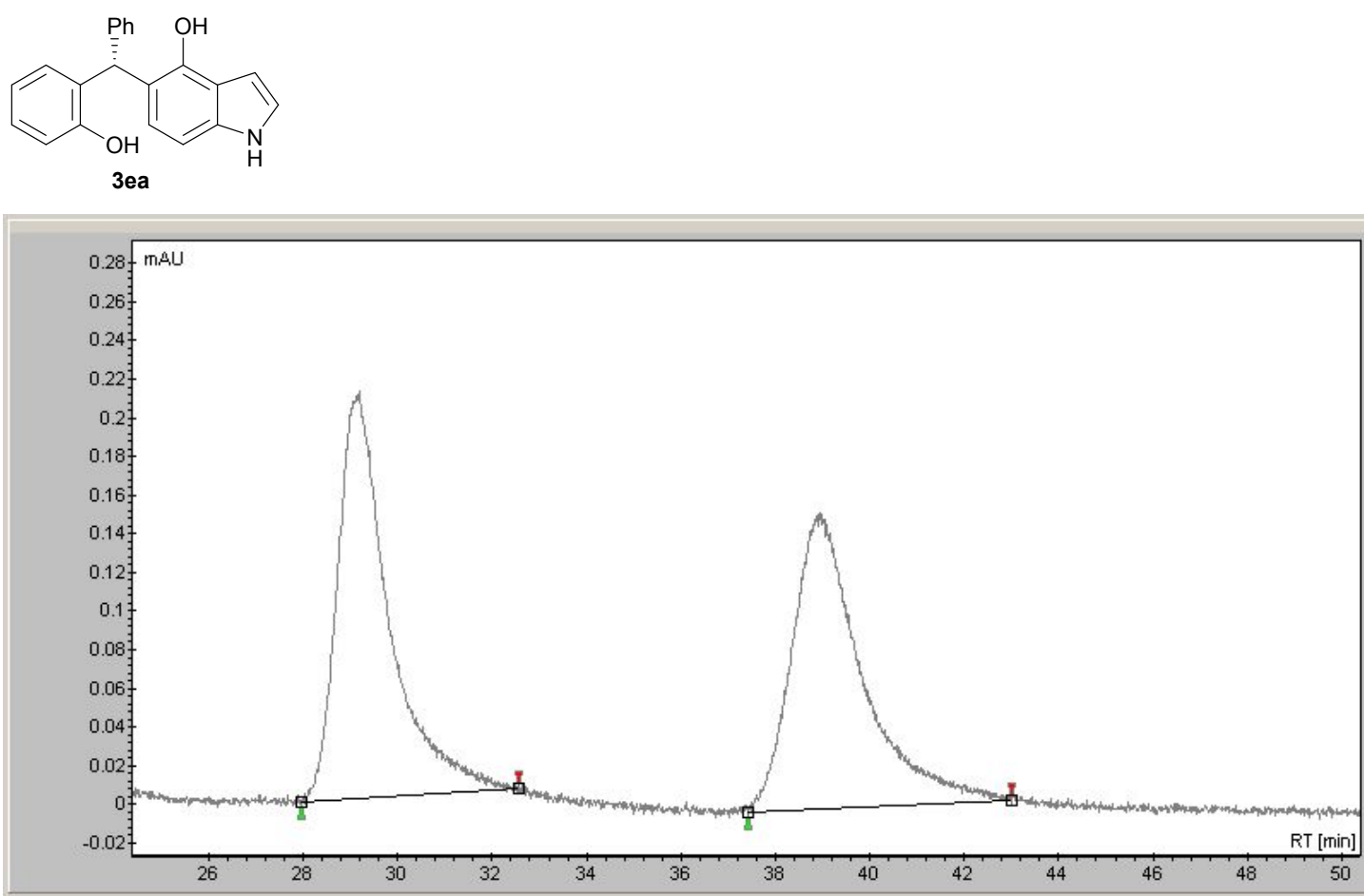

\begin{tabular}{|r|r|r|r|r|r|r|r|r|r|r|}
\hline$\#$ & Name & Start [Min] & Time [Min] & End [Min] & Ret. time Offset & Quantity [\% Arei & Height [mAU] & Area [mAU.Min] & Area\% [\%] \\
\hline 1 & UNKNOWN & 27.961 & 29.118 & 32.565 & 0.000 & 50.05 & 0.2 & 0.3 & 50.046 \\
\hline 2 & UNKNOWN & 37.424 & 38.917 & 43.007 & 0.000 & 49.95 & 0.2 & 0.3 & 49.954 \\
\hline & & & & & & & 100.00 & 0.4 & 0.5 & 100.000 \\
\hline
\end{tabular}

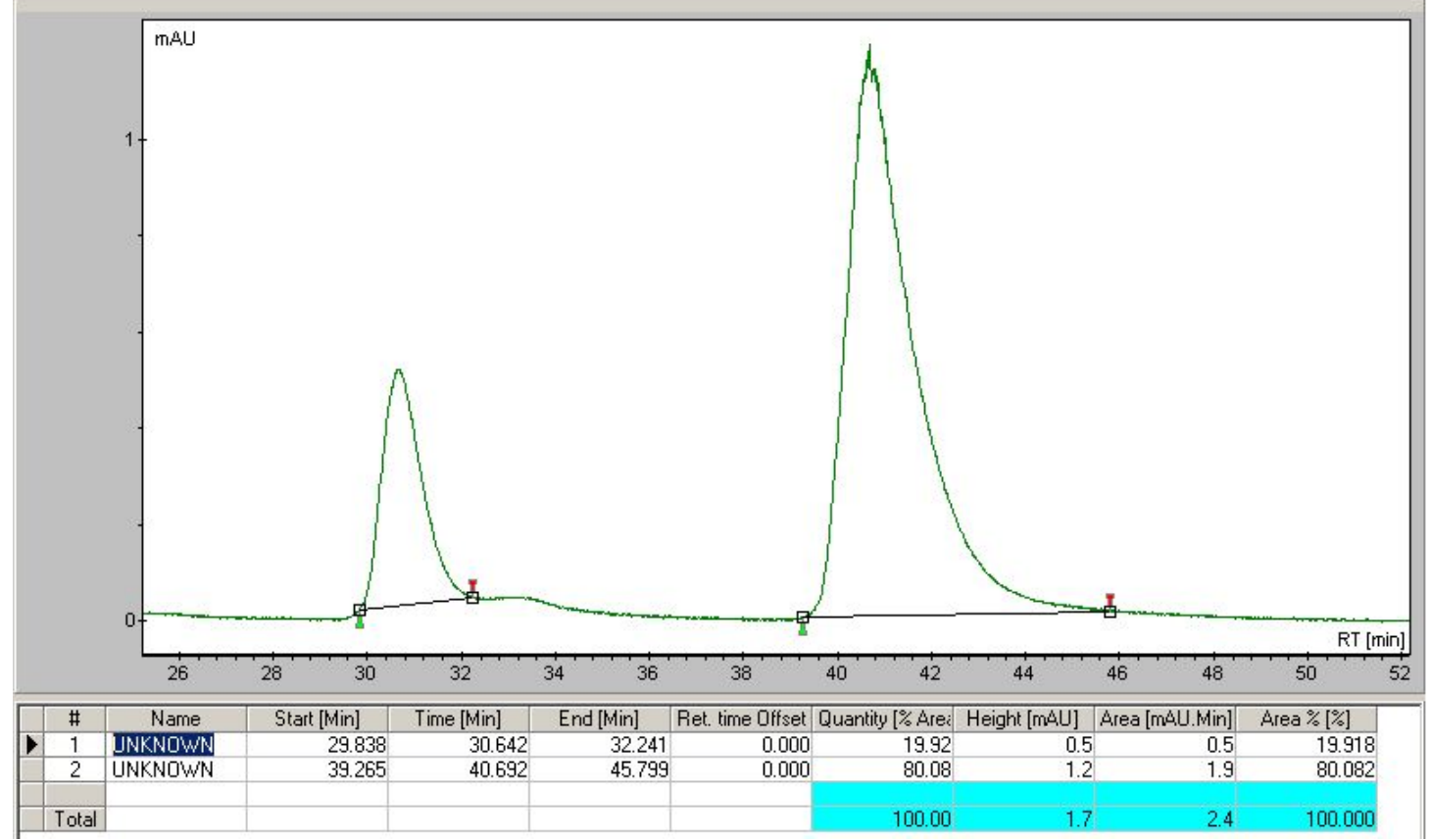



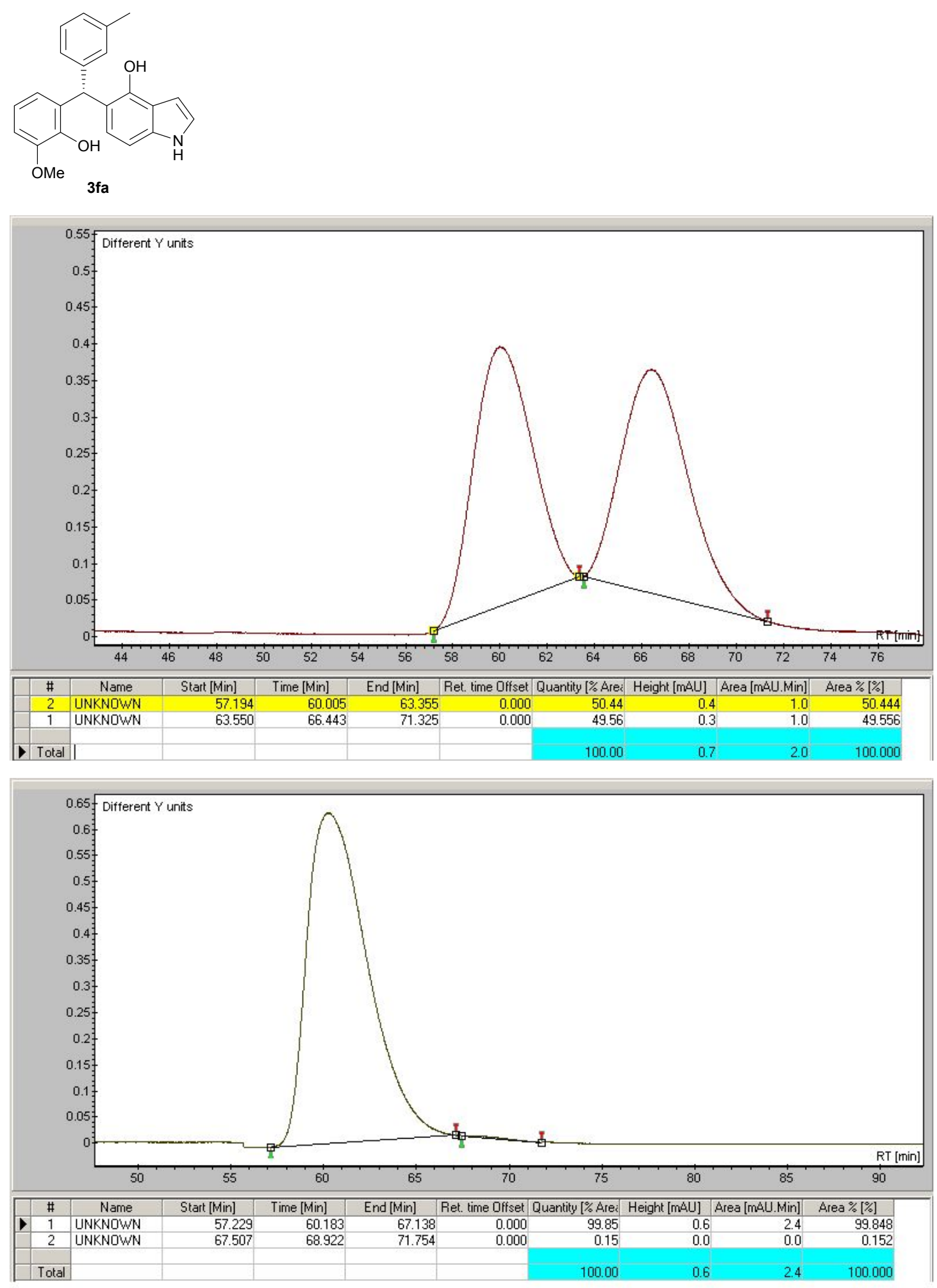
<smiles>COc1cccc([C@H](c2ccc(C)cc2)c2ccc3[nH]ccc3c2O)c1O</smiles>
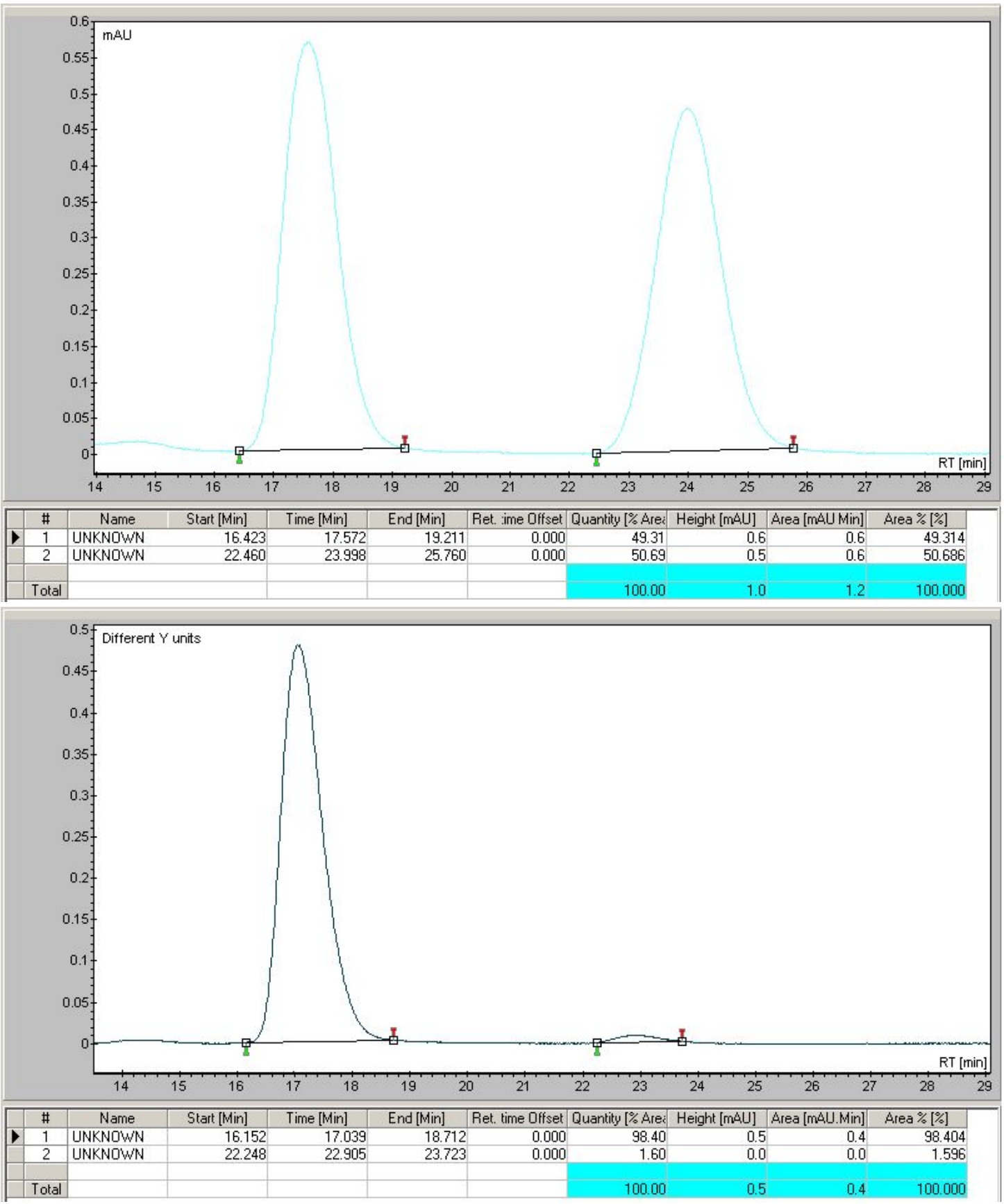
<smiles>COc1cccc([C@H](c2cccc(OC)c2O)c2ccc3[nH]ccc3c2O)c1</smiles>

3ha
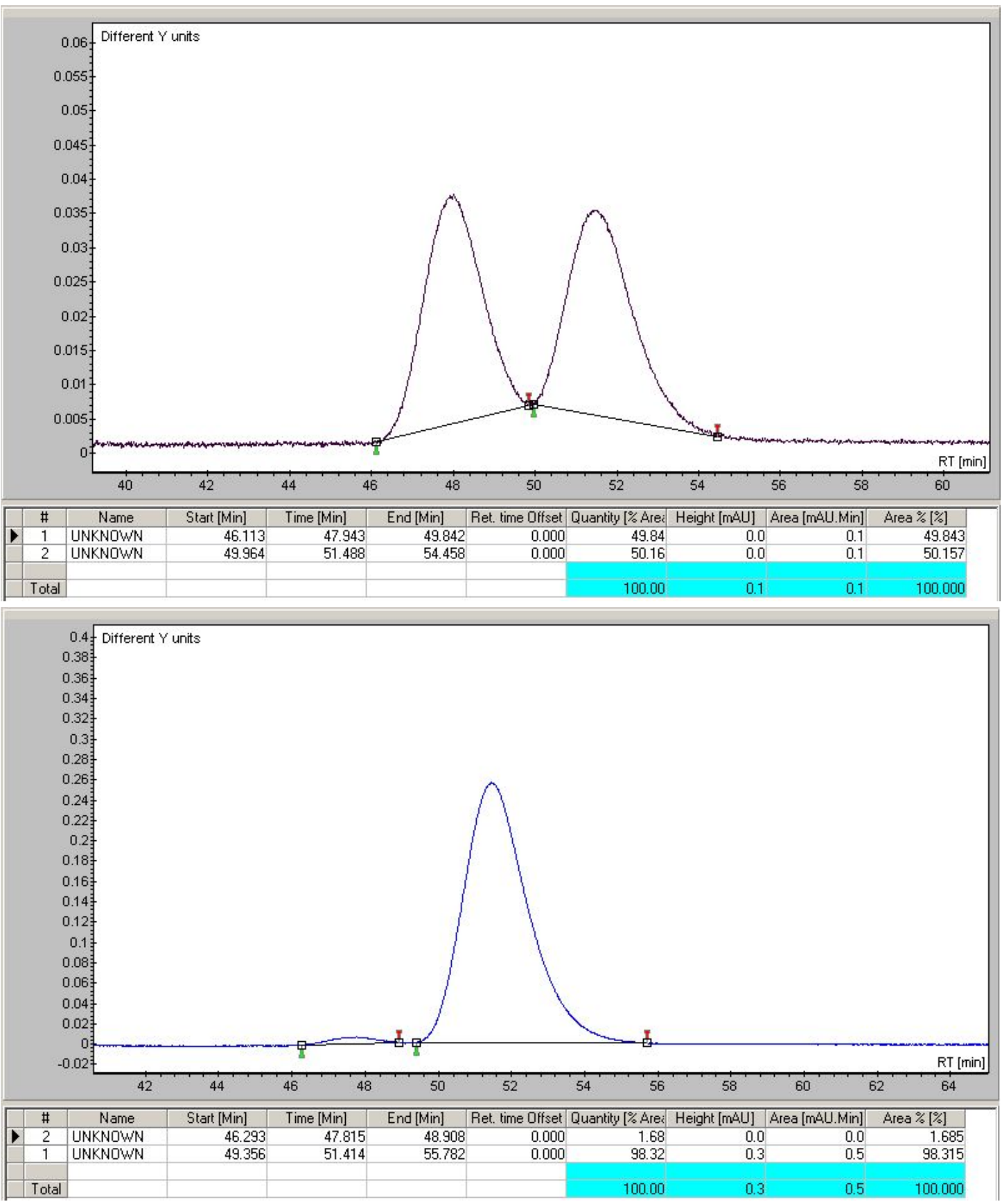
<smiles>COc1cccc([C@H](c2cccc(OC(F)(F)F)c2)c2ccc3[nH]ccc3c2O)c1O</smiles>
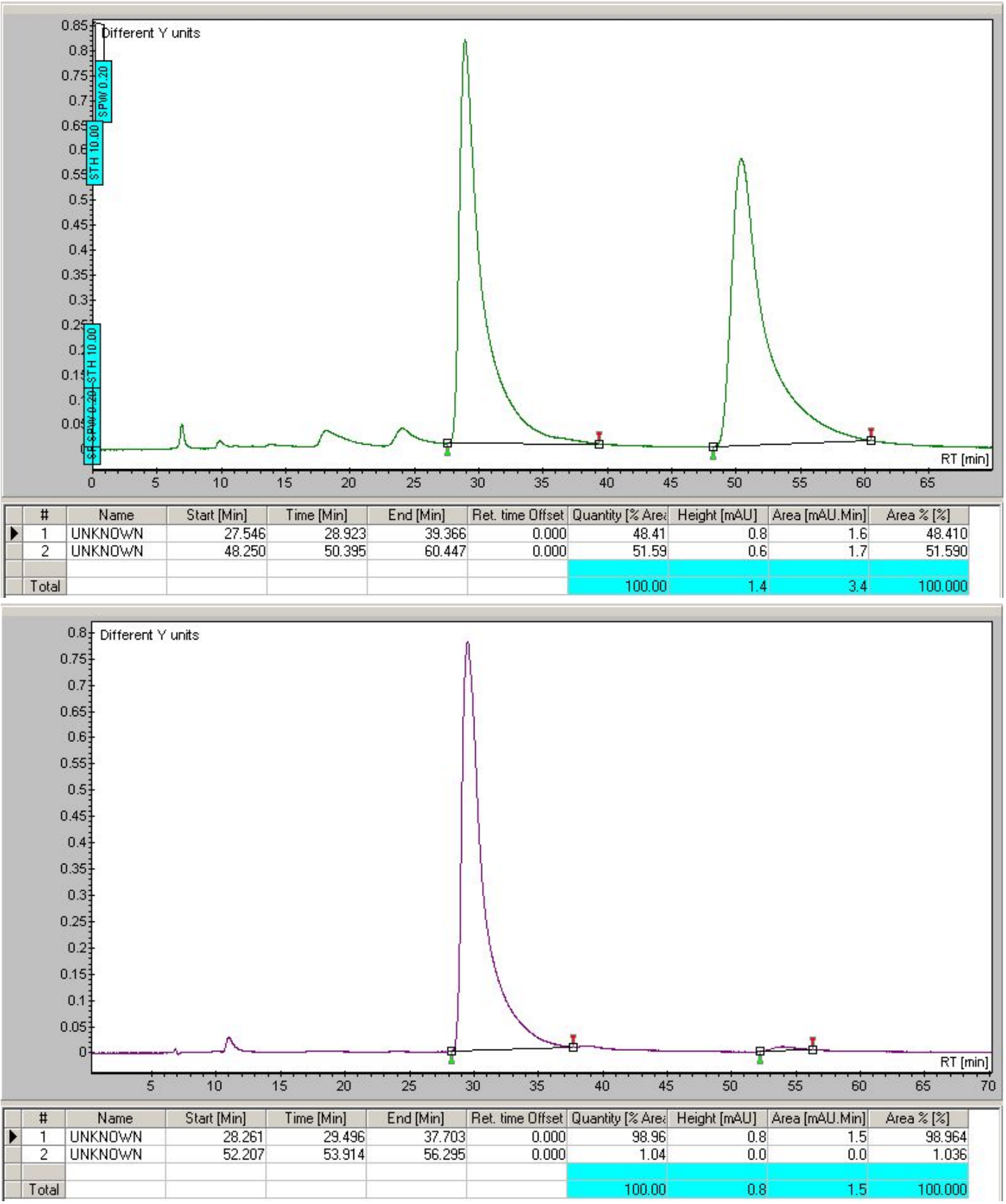
<smiles>COc1cccc([C@H](c2ccc(OC(F)(F)F)cc2)c2ccc3[nH]ccc3c2O)c1O</smiles>
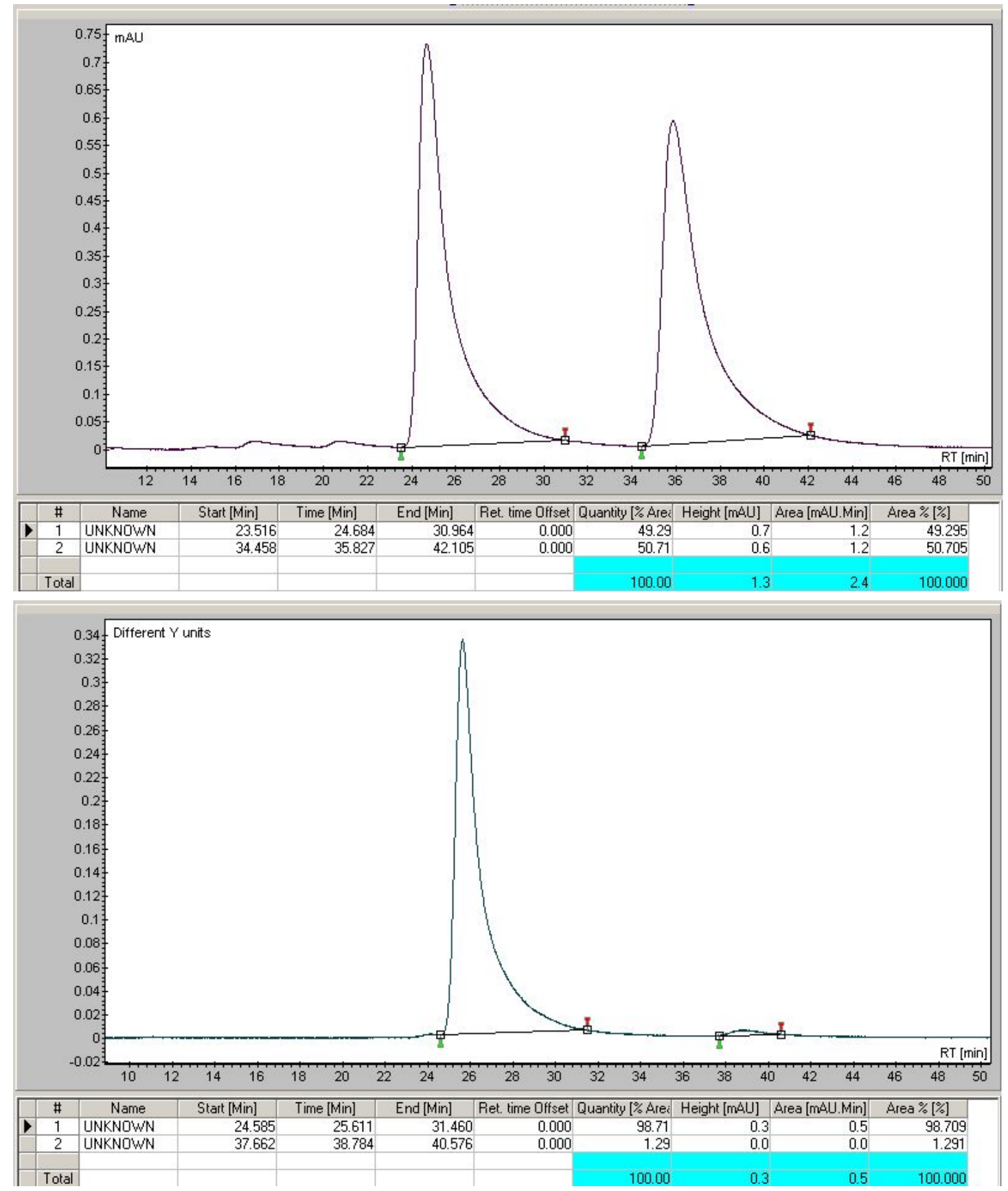
<smiles>COc1cccc(C(c2ccc(F)cc2)c2ccc3[nH]ccc3c2O)c1O</smiles>

3ka

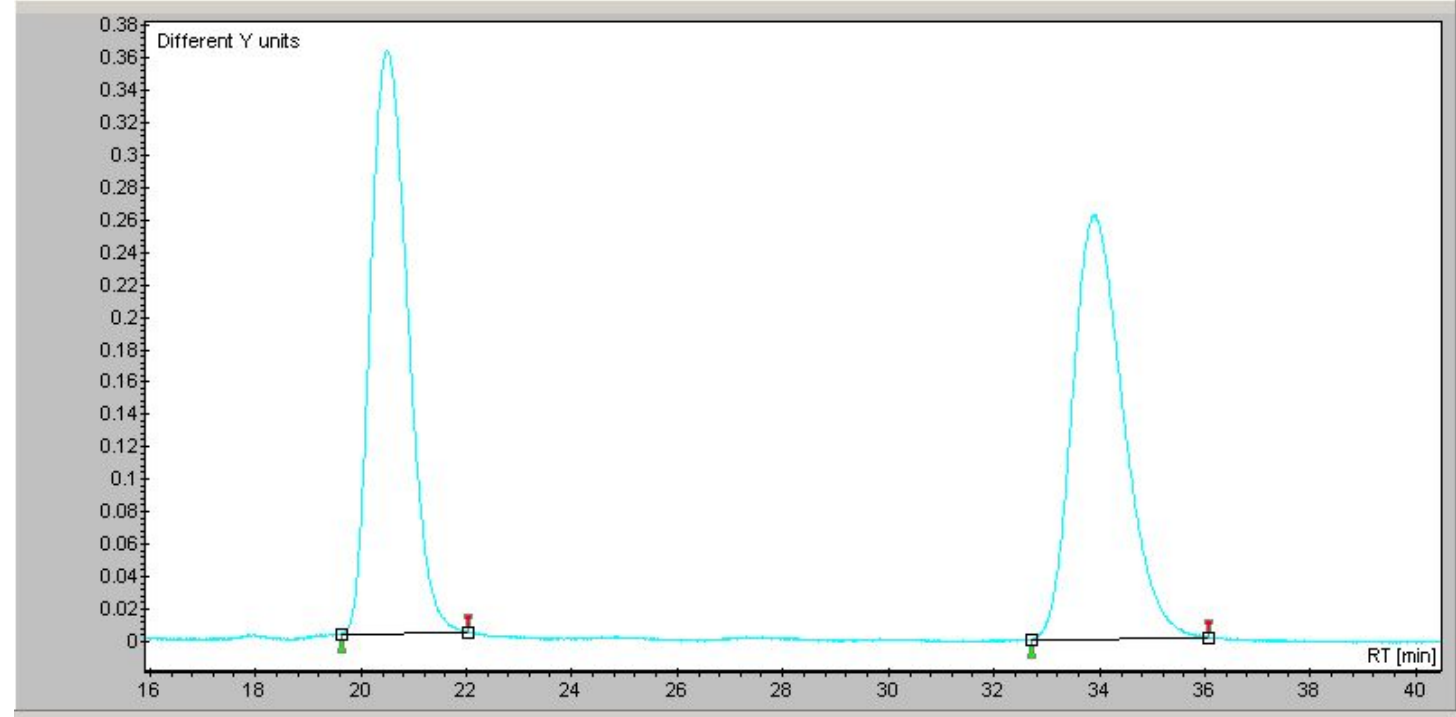

\begin{tabular}{|c|c|c|c|c|c|c|c|c|c|c|}
\hline & $\#$ & Name & Start [Min] & Time [Min] & End [Min] & Ret. time Offset & Quantity [\% Arei & Height [mAU] & Area [mAU.Min] & Area \% [\%] \\
\hline 1 & 1 & UNKNOWN & 19.636 & 20.492 & 22.032 & 0.000 & 50.23 & 0.4 & 0.3 & 50.235 \\
\hline & 2 & UNKNOWN & 32.712 & 33.891 & 36.067 & 0.000 & 49.77 & 0.3 & 0.3 & 49.765 \\
\hline & Total & & & & & & 100.00 & 0.6 & 0.6 & 100.000 \\
\hline
\end{tabular}

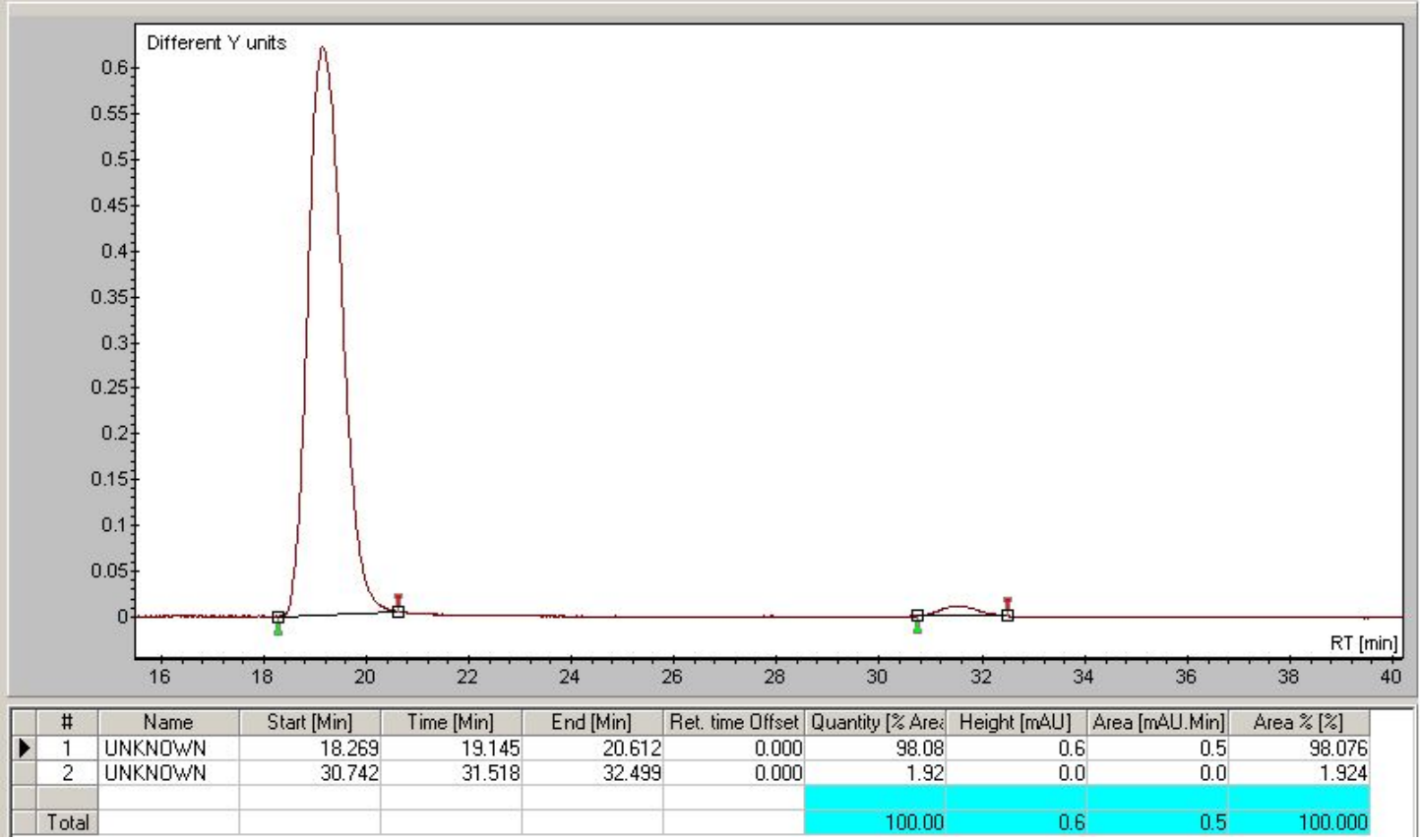


<smiles>COc1cccc([C@@H](c2ccc(Cl)cc2)c2ccc3[nH]ccc3c2O)c1O</smiles>
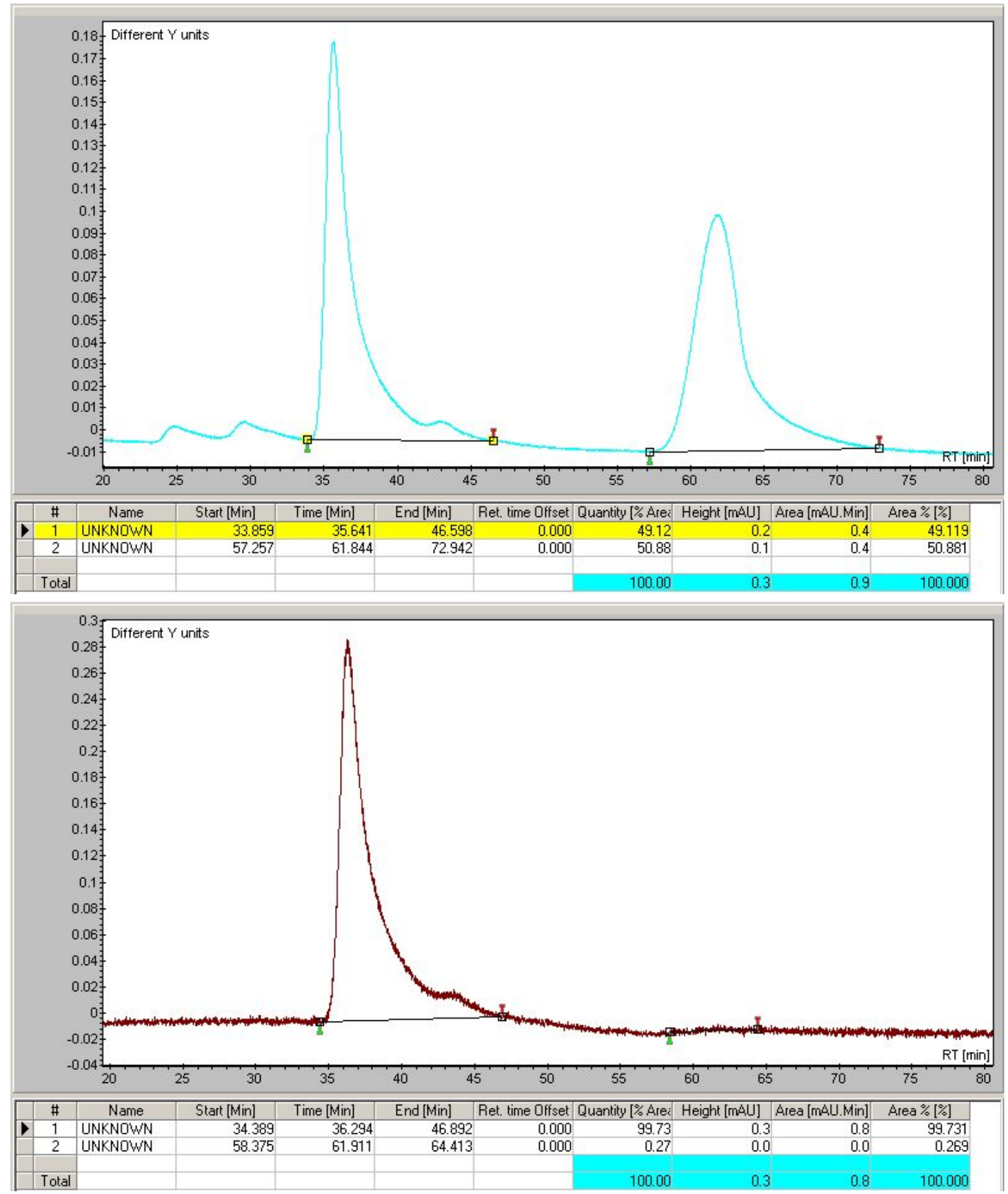
<smiles>COc1cccc([C@H](c2ccccc2C(F)(F)F)c2ccc3[nH]ccc3c2O)c1O</smiles>
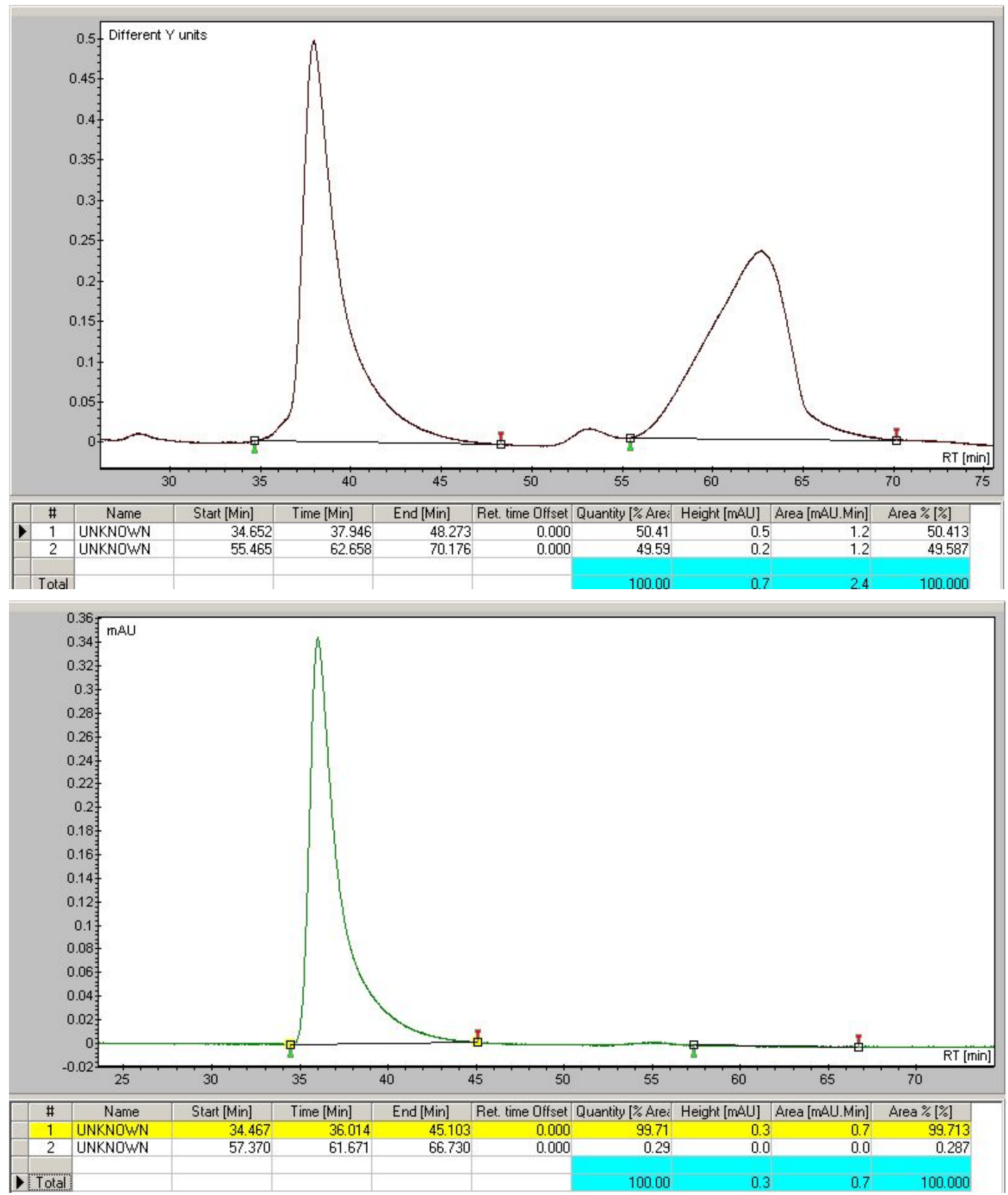
<smiles>COc1cccc(C(c2cccc(C(F)(F)F)c2)c2ccc3[nH]ccc3c2O)c1O</smiles>

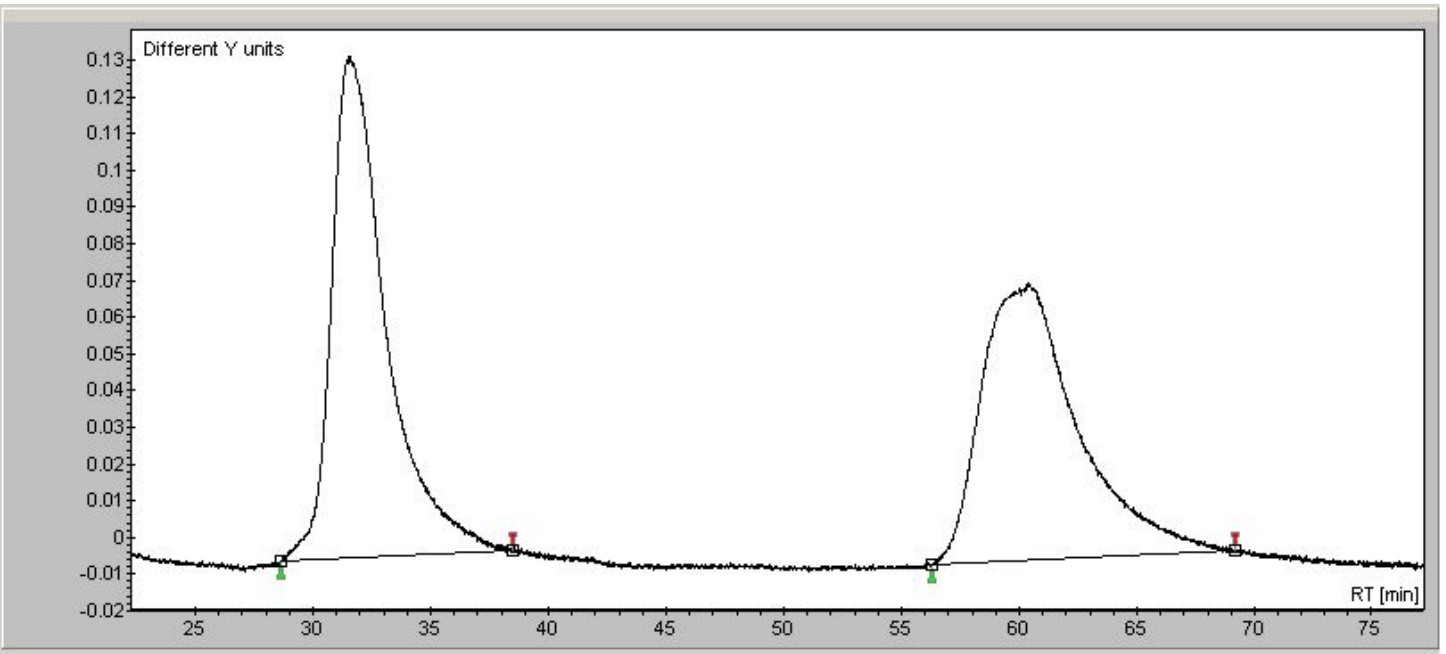

\begin{tabular}{|c|c|c|c|c|c|c|c|c|c|c|}
\hline & \# & Name & Start [Min] & Time [Min] & End [Min] & Ret. time Offset & Quantity [\% Arei & Height [mAU] & Area [mAU.Min] & Area \% [\%] \\
\hline 1 & 2 & UNKNOWN & 28.636 & 31.562 & $38.49 \pi$ & 0.000 & 50.58 & 0.1 & 0.4 & 50.580 \\
\hline & 1 & UNKNOWN & 56.312 & 60.432 & 69.202 & 0.000 & 49.42 & 0.1 & 0.4 & 49.420 \\
\hline & Total & & & & & & 100.00 & 0.2 & 0.7 & 100.000 \\
\hline
\end{tabular}

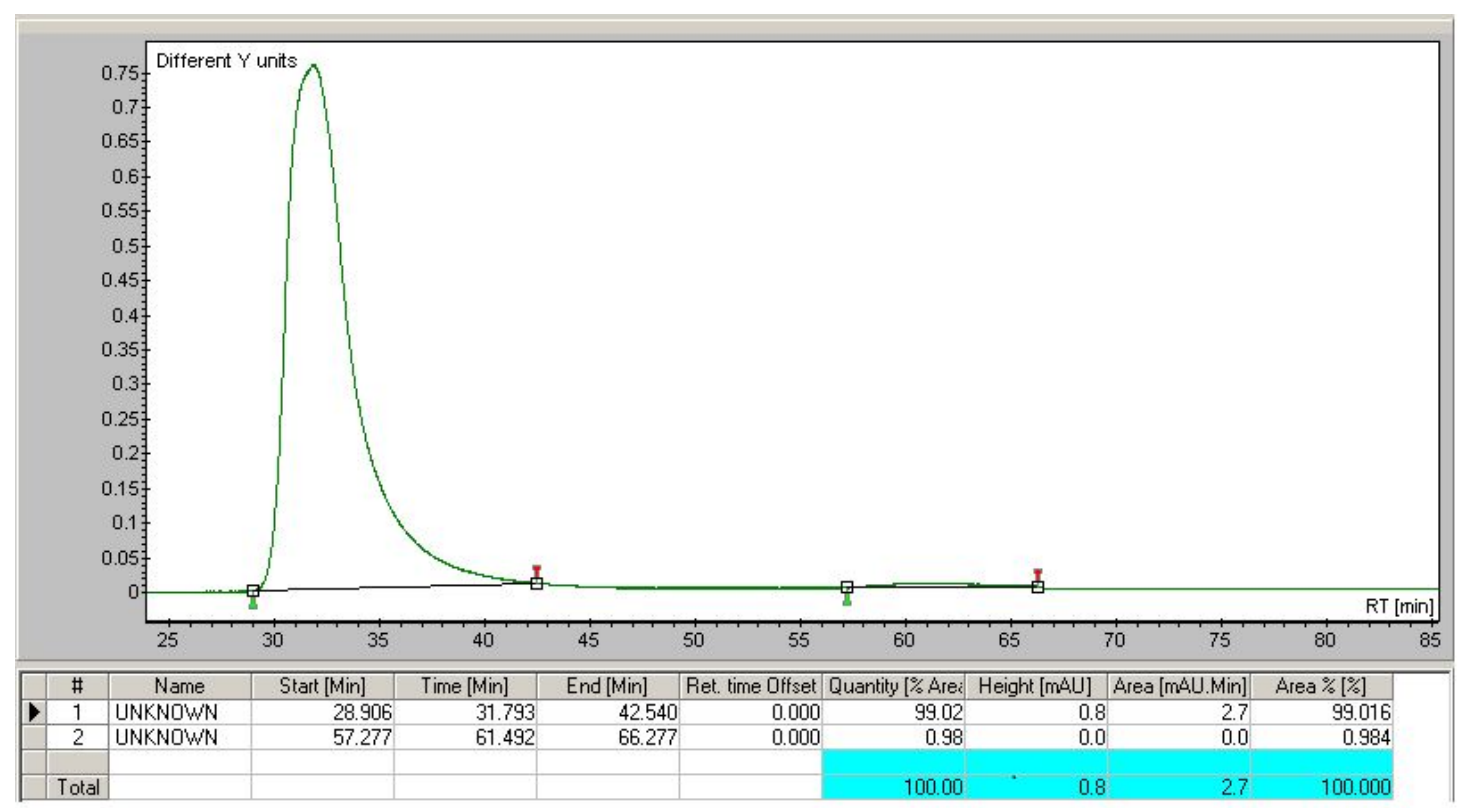


<smiles>COc1cccc([C@H](c2ccc(C(F)(F)F)cc2)c2ccc3[nH]ccc3c2O)c1O</smiles>

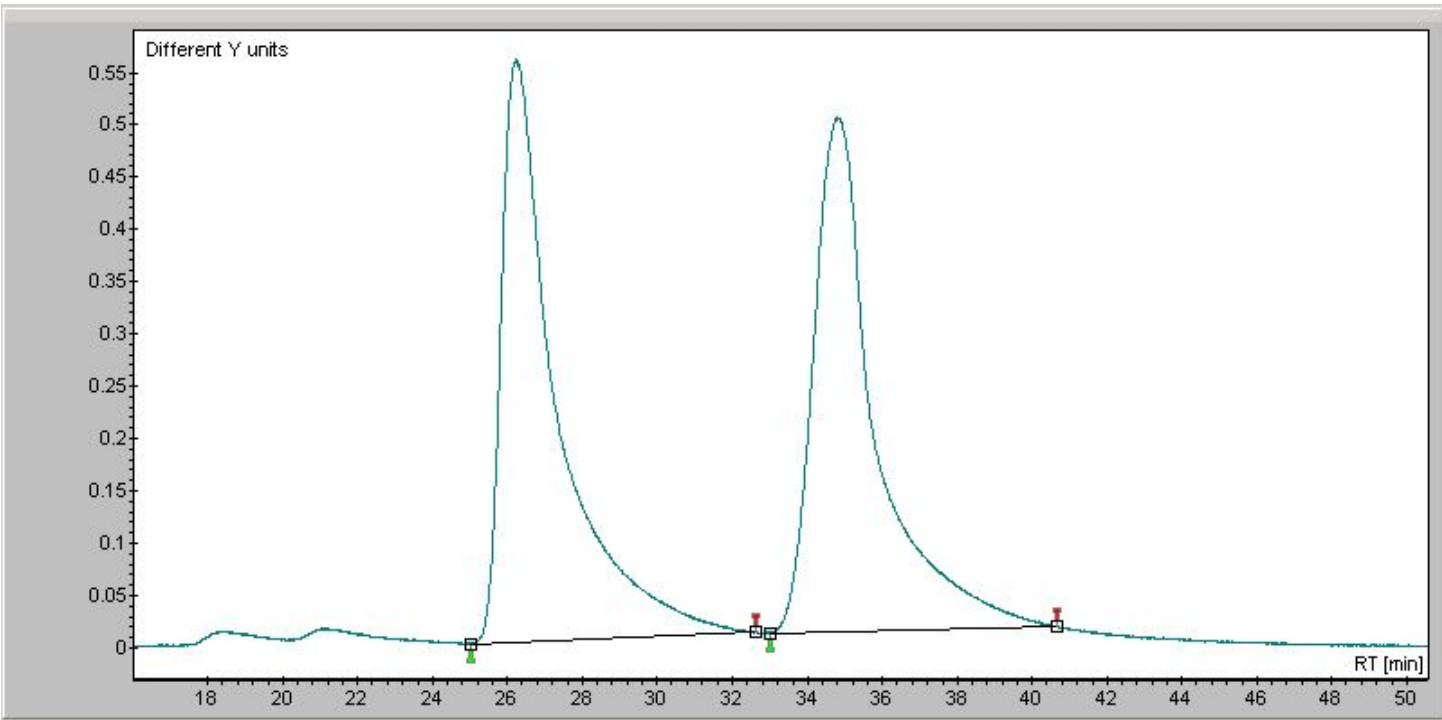

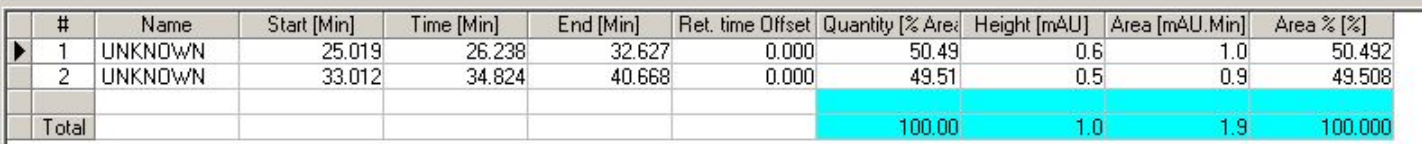

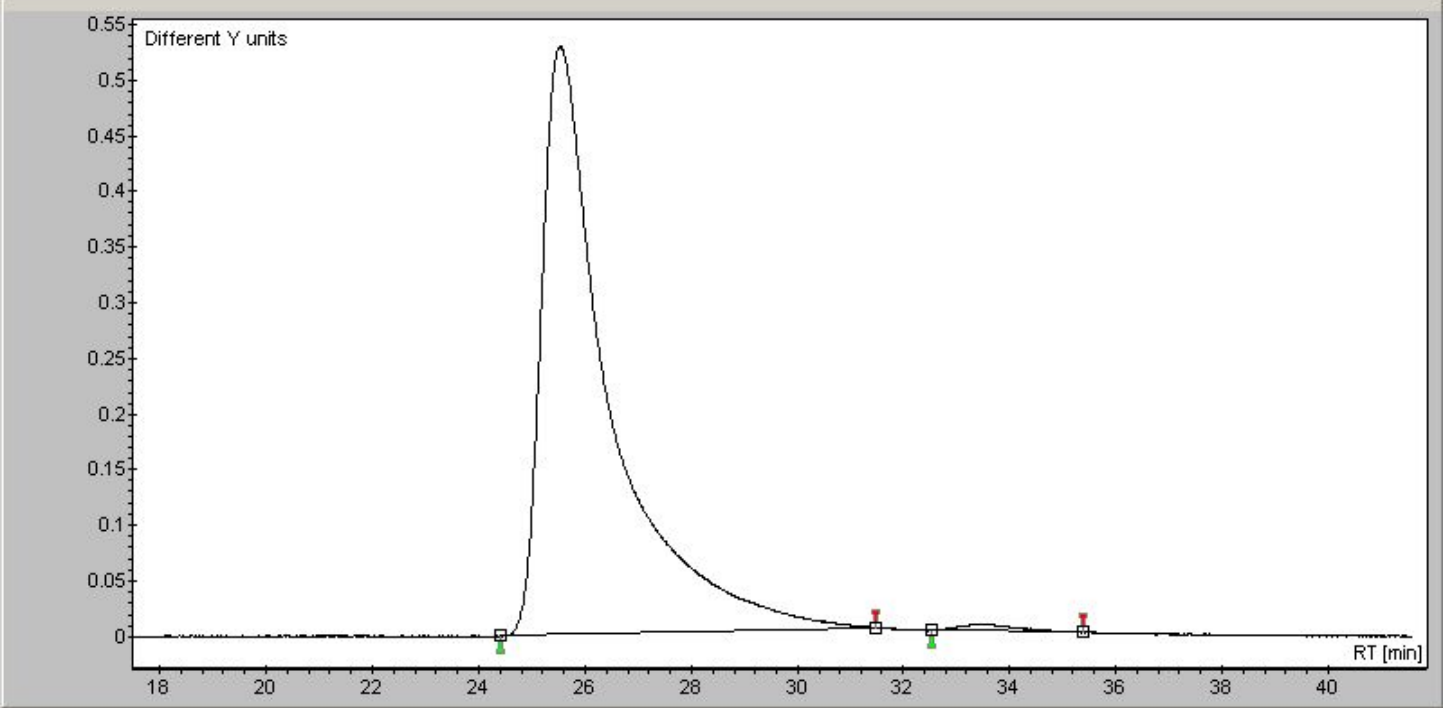

\begin{tabular}{|c|c|c|c|c|c|c|c|c|c|}
\hline \# & Name & Start [Min] & Time [Min] & End [Min] & Ret. time Offset & Quantity [\% Aree & Height [mAU] & Area [mA.U.Min] & Area \% [\%] \\
\hline 1 & UNKNOWN & 24.415 & 25.545 & 31.460 & 0.000 & $\quad 99.10$ & 0.5 & $\quad 0.8$ & 99.099 \\
\hline 2 & UNKNOWN & 32.541 & 33.464 & 35.378 & 0.000 & 0.90 & 0.0 & 0.0 & 0.901 \\
\hline Total & & & & & & 100.00 & 0.5 & 0.8 & 100.000 \\
\hline
\end{tabular}


<smiles>COc1cccc([C@@H](c2ccc(-c3ccccc3)cc2)c2ccc3[nH]ccc3c2O)c1</smiles>

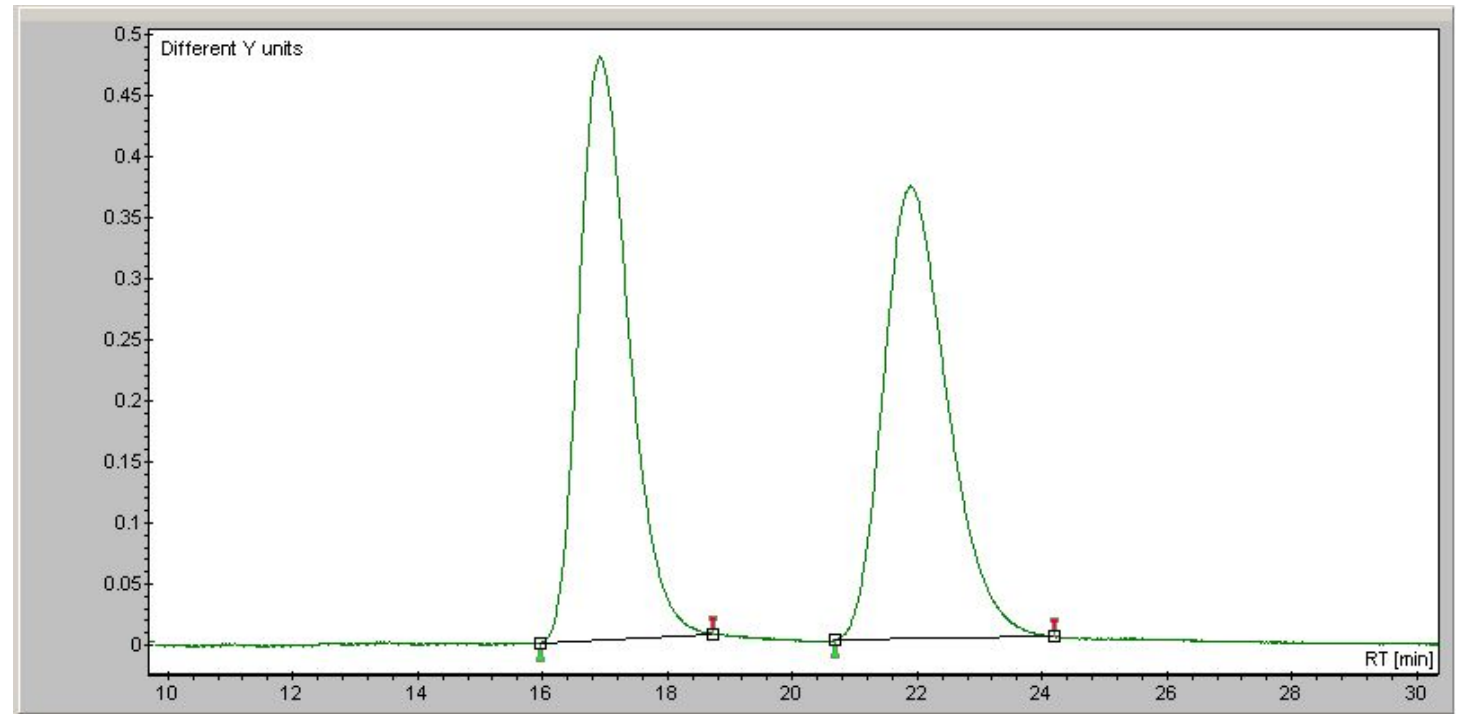

\begin{tabular}{|c|c|c|c|c|c|c|c|c|c|c|}
\hline & $\#$ & Name & Start [Min] & Time [Min] & End [Min] & Ret. time Offset & Quantity [\% Arei & Height [mAU] & Area [mAU.Min] & Area \% [\%] \\
\hline 1 & & UNKNOW/N & 15.967 & 16.919 & 18.730 & 0.000 & 50.01 & 0.5 & 0.4 & 50.014 \\
\hline & 2 & UNKNOWN & 20.687 & 21.892 & 24.199 & 0.000 & 49.99 & 0.4 & 0.4 & 49.986 \\
\hline & Total & & & & & & 100.00 & 0.8 & 0.9 & 100.000 \\
\hline
\end{tabular}

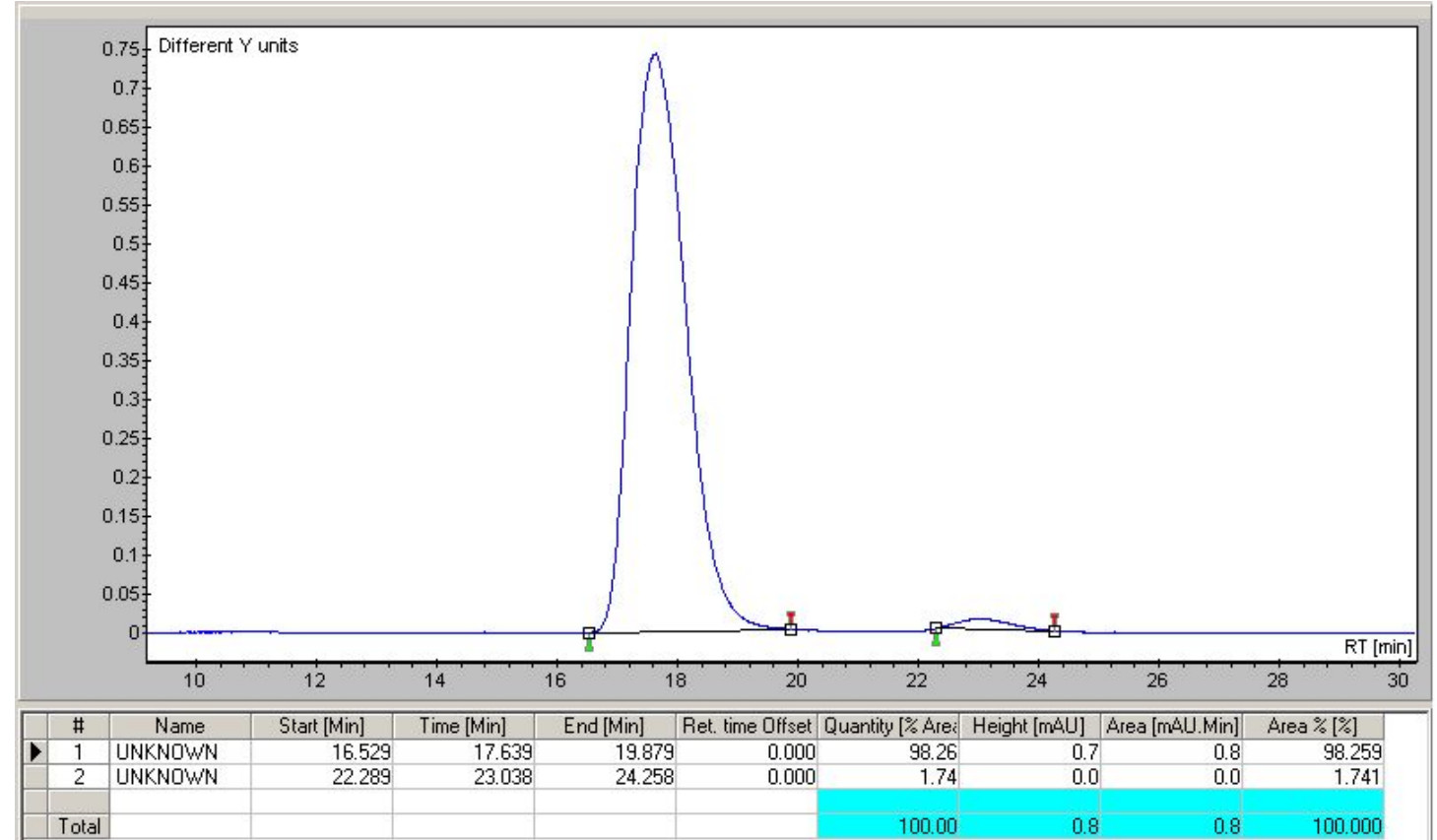




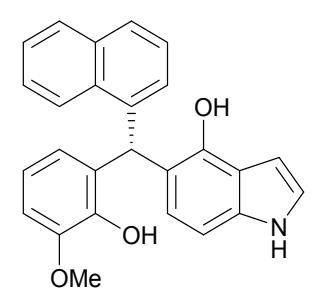

$3 q a$
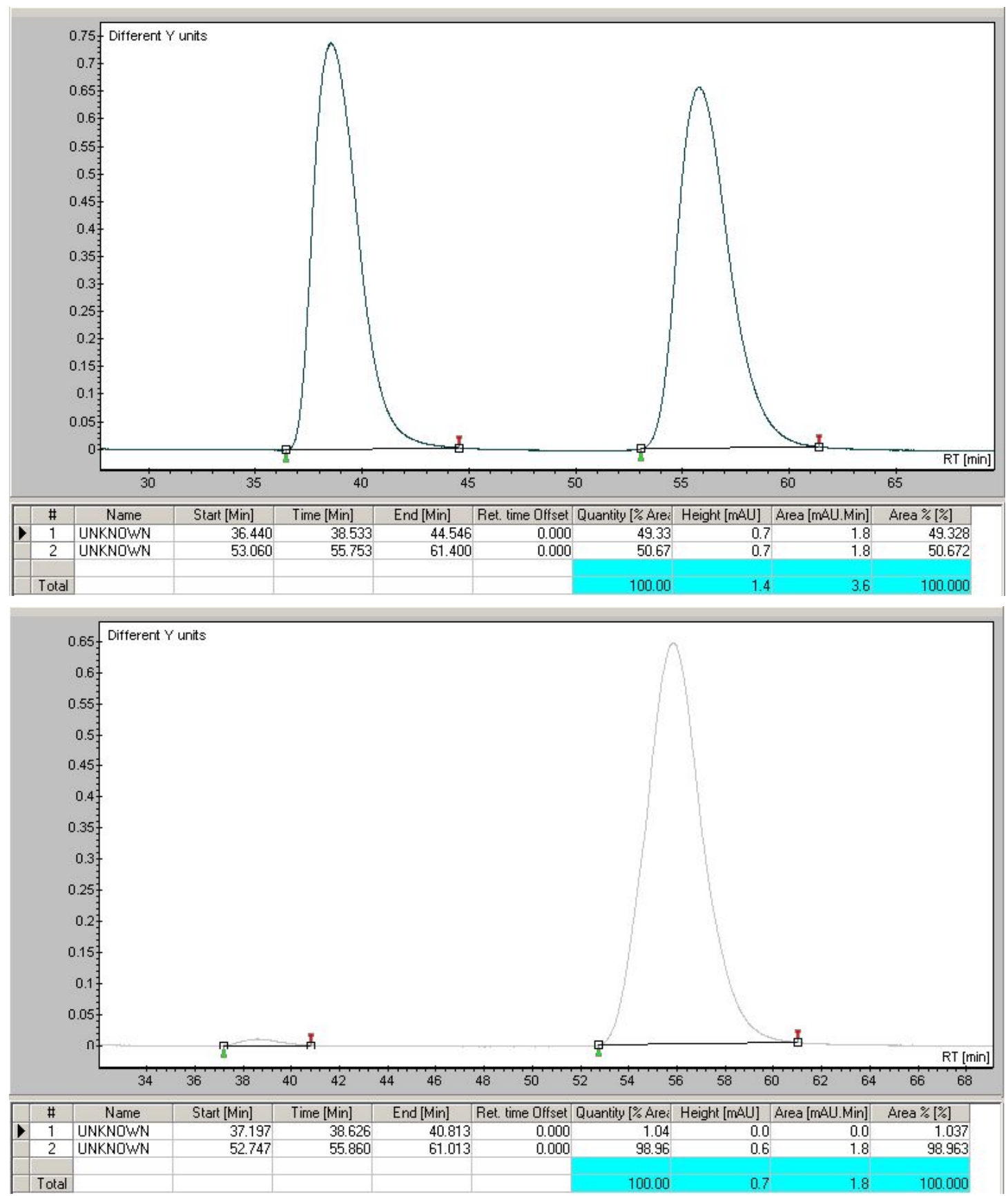
<smiles>COc1cccc(C(c2ccc3ccccc3c2)c2ccc3[nH]ccc3c2O)c1O</smiles>
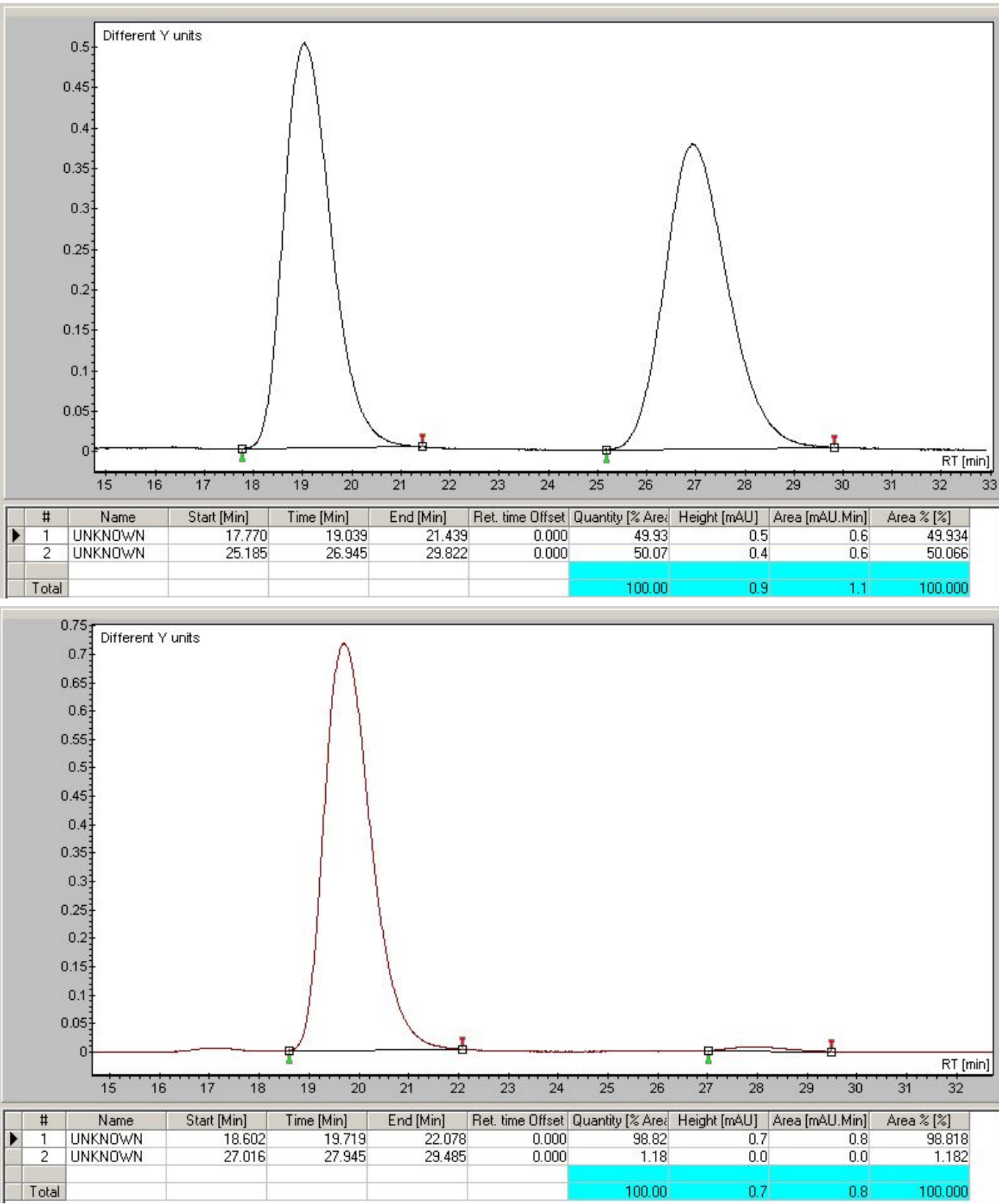

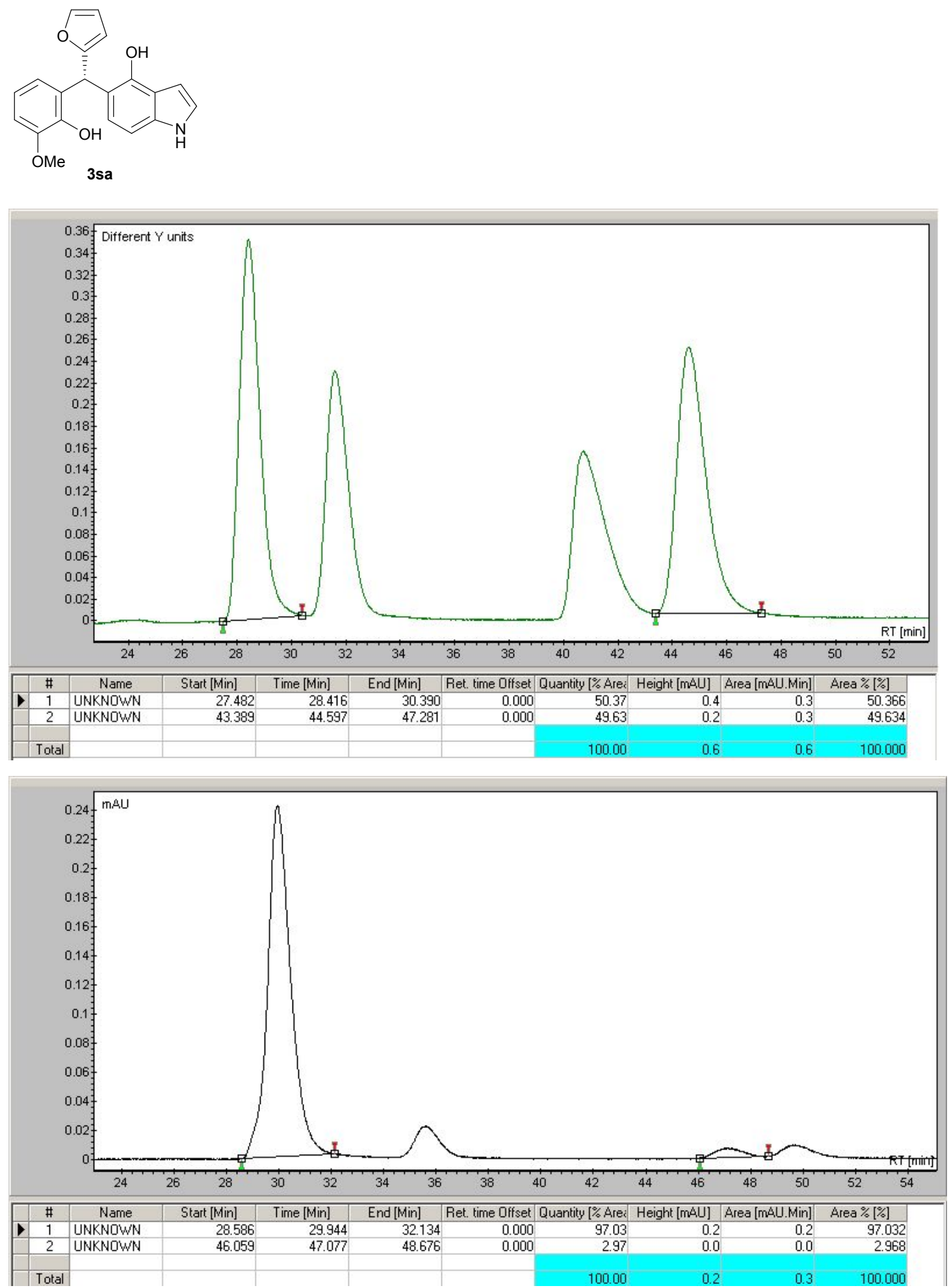
<smiles>COc1cccc([C@@H](c2cccs2)c2ccc3[nH]ccc3c2O)c1O</smiles>
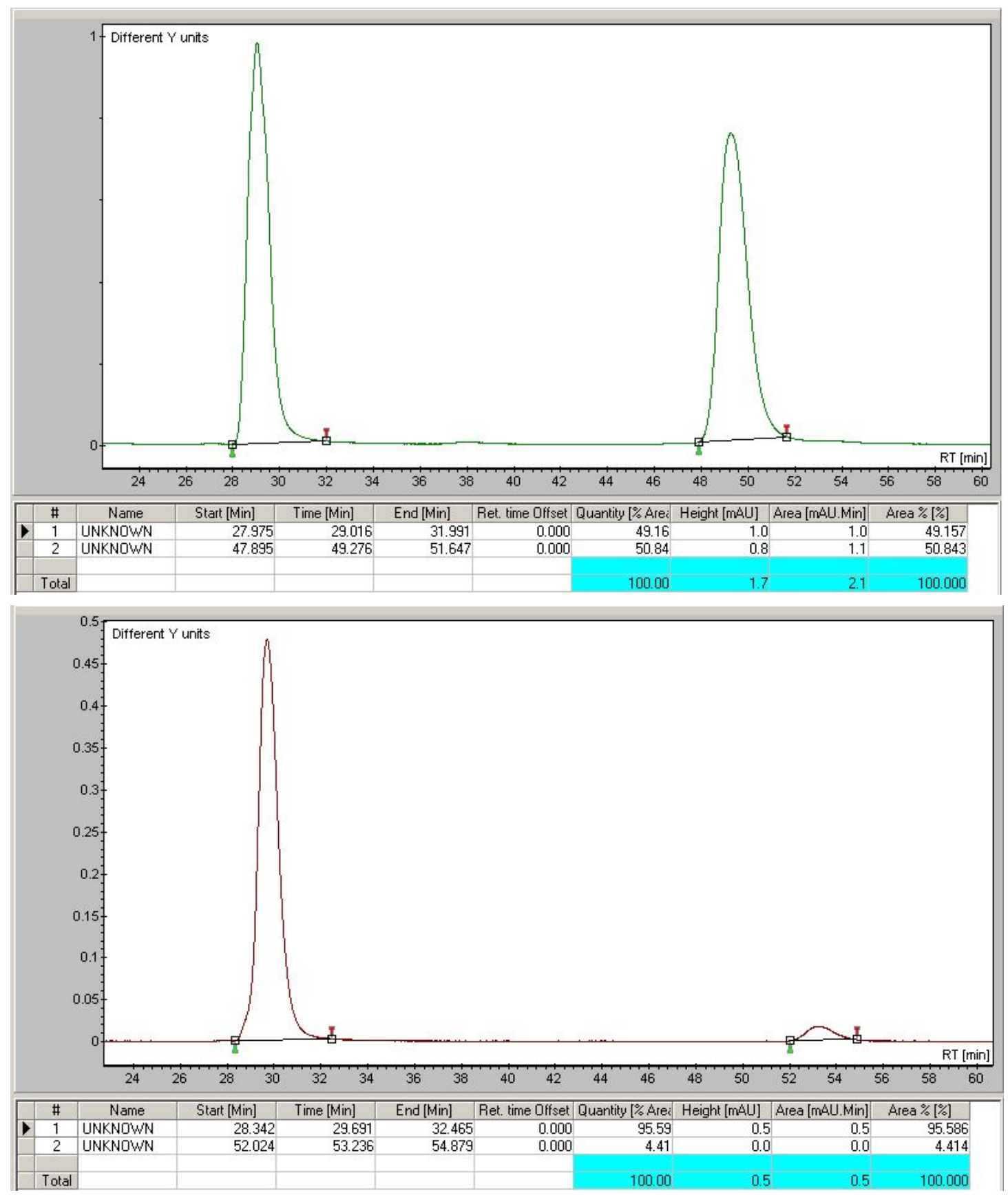
<smiles>COc1cccc(C(c2ccccc2)c2c(O)ccc3[nH]ccc23)c1O</smiles>

$3 a b$

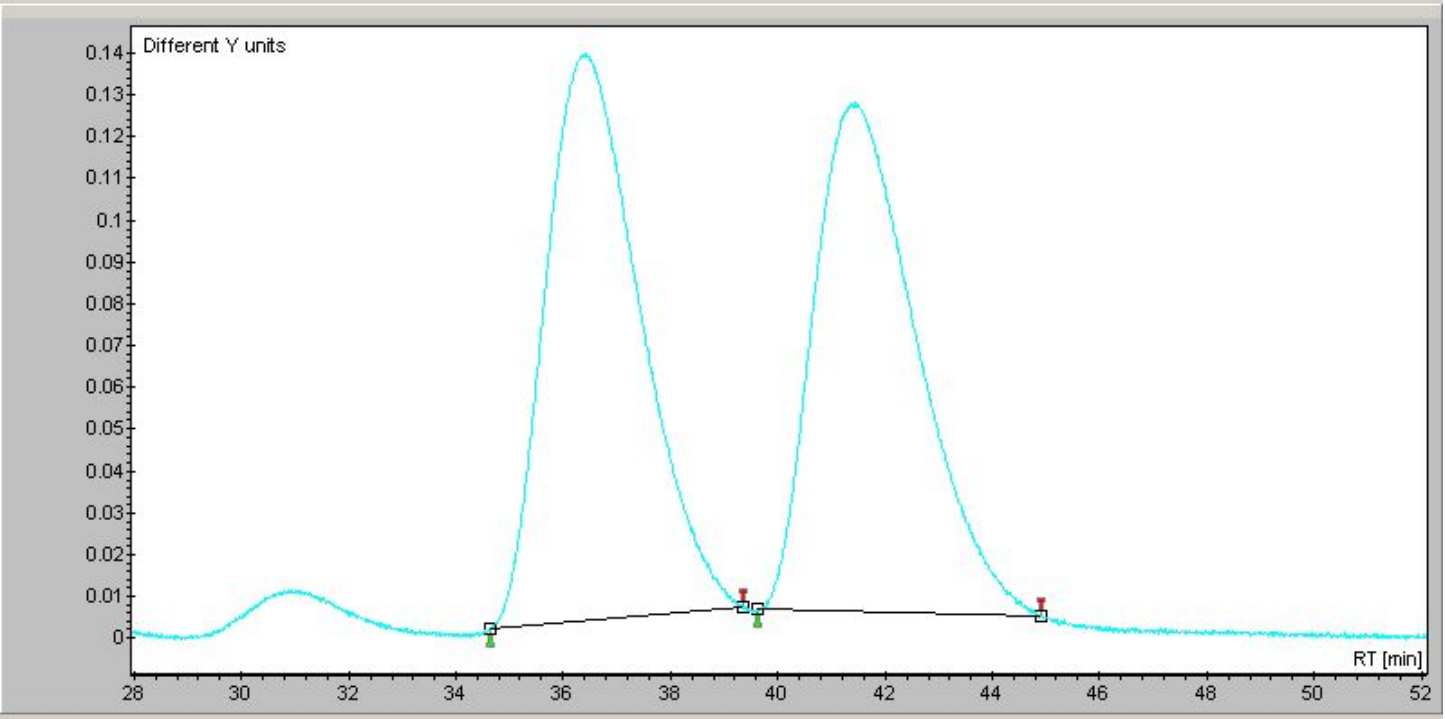

\begin{tabular}{|c|c|c|c|c|c|c|c|c|c|c|}
\hline & \# & Name & Start [Min] & Time [Min] & End [Min] & Ret. time Offset & Quantity [\% Are: & Height [mAU] & Area [mAU.Min] & Area \% [\%] \\
\hline D & 1 & UNKNOWN & 34.639 & 36.397 & 39.352 & $\quad 0.000$ & 50.60 & 0.1 & 0.3 & 50.604 \\
\hline & 2 & UNKNOWN & 39.622 & 41.424 & 44.907 & 0.000 & 49.40 & 0.1 & 0.3 & 49.396 \\
\hline & Total & & & & & & 100.00 & 03 & 0.5 & 100.000 \\
\hline
\end{tabular}

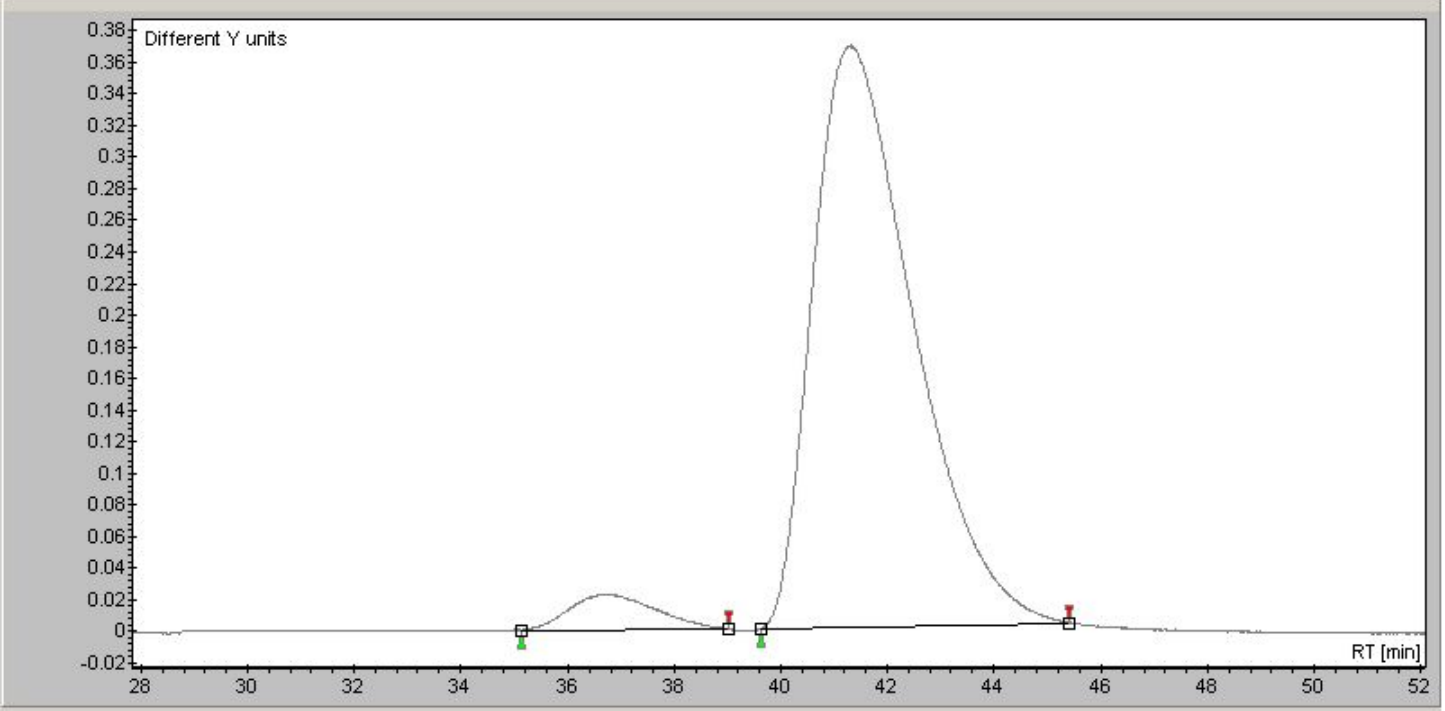

\begin{tabular}{|c|c|c|c|c|c|c|c|c|c|c|}
\hline & $\#$ & Name & Start [Min] & Time [Min] & End [Min] & Ret. time Offset & Quantity [\% Are: & Height [mAU] & Area [mAU.Min] & Area $\%[\%]$ \\
\hline D & 1 & UNKNOWN & 35.138 & 36.694 & 39.023 & 0.000 & 4.96 & 0.0 & 0.0 & 4.956 \\
\hline & 2 & UNKNOWN & 39.631 & 41.305 & 45.407 & 0.000 & 95.04 & 0.4 & 0.8 & 95.044 \\
\hline & Total & & & & & & 100.00 & 0.4 & 0.8 & 100.000 \\
\hline
\end{tabular}


<smiles>COc1cccc(C(c2cccc(C)c2)c2c(O)ccc3[nH]ccc23)c1O</smiles>

$3 b b$
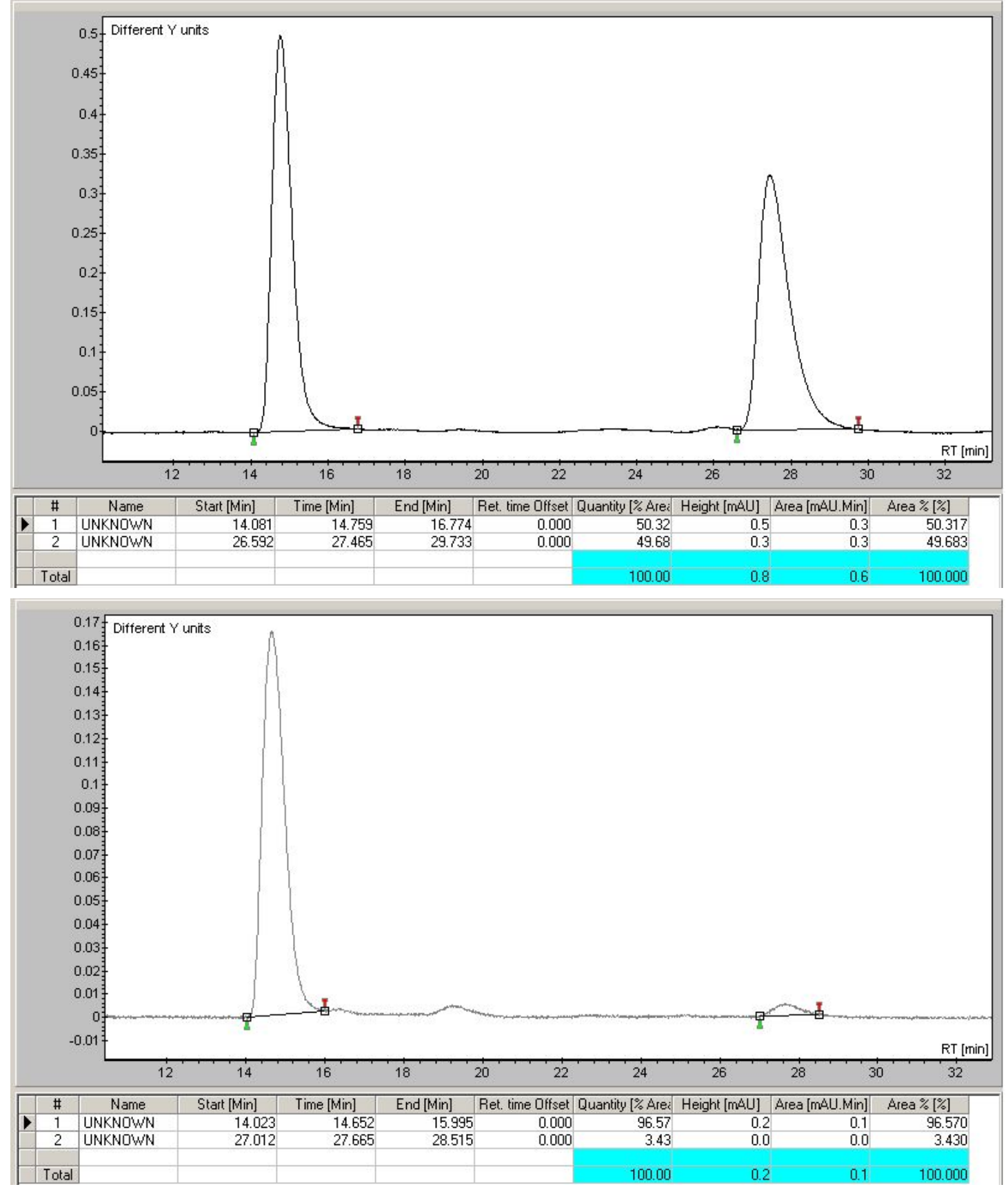

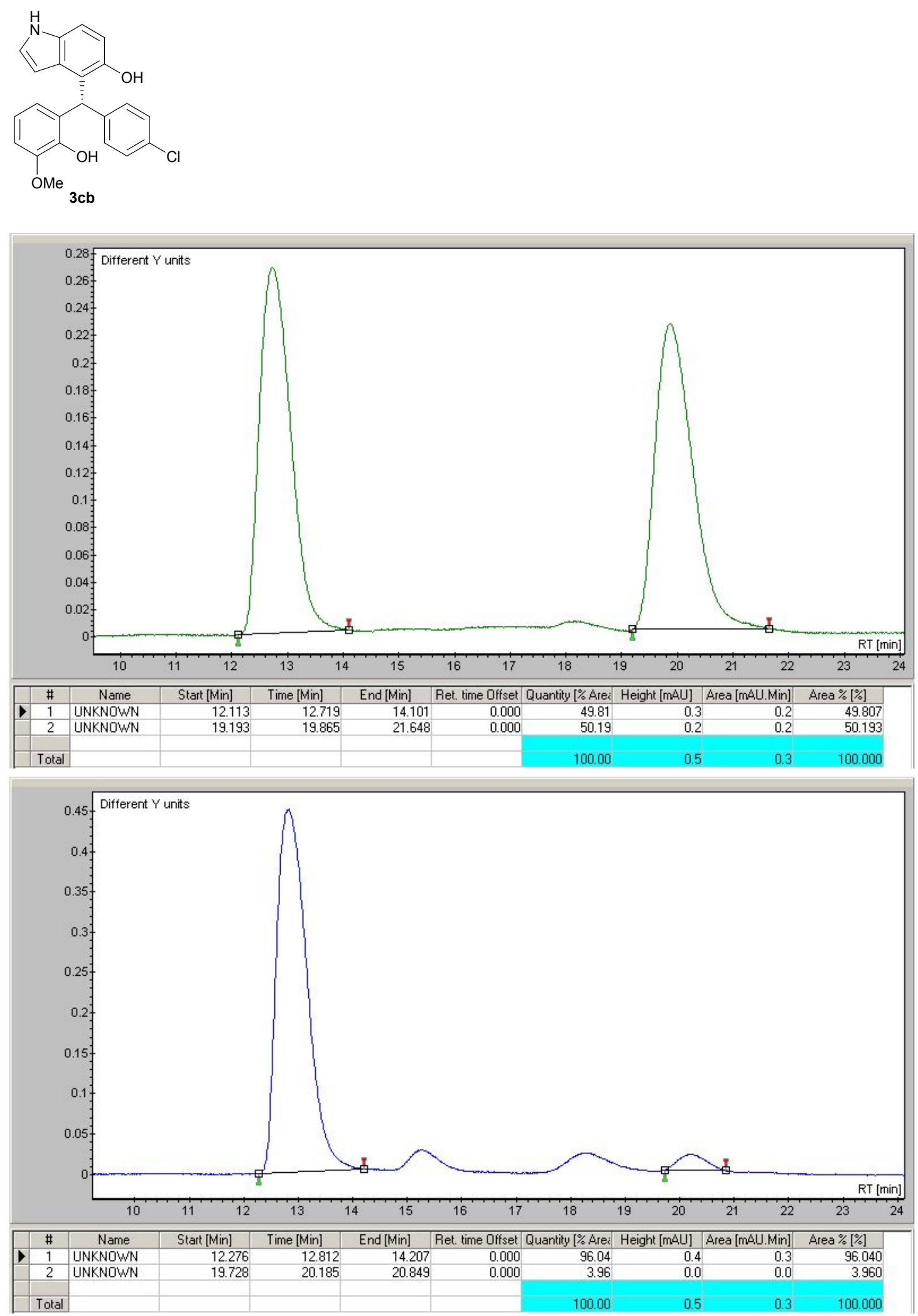
<smiles>COc1cccc(C(c2ccccc2)c2ccc3cc[nH]c3c2O)c1O</smiles>
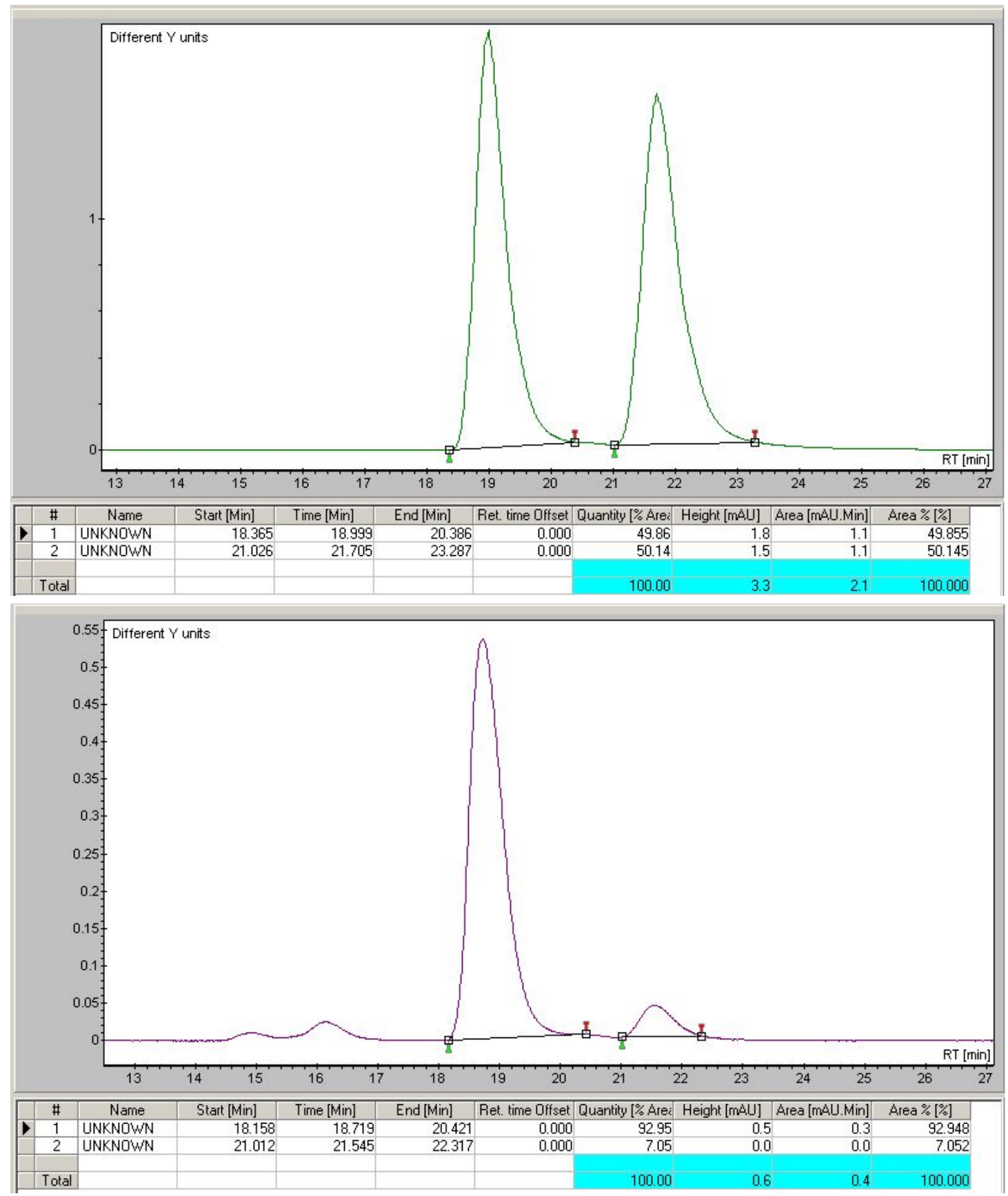
<smiles></smiles>
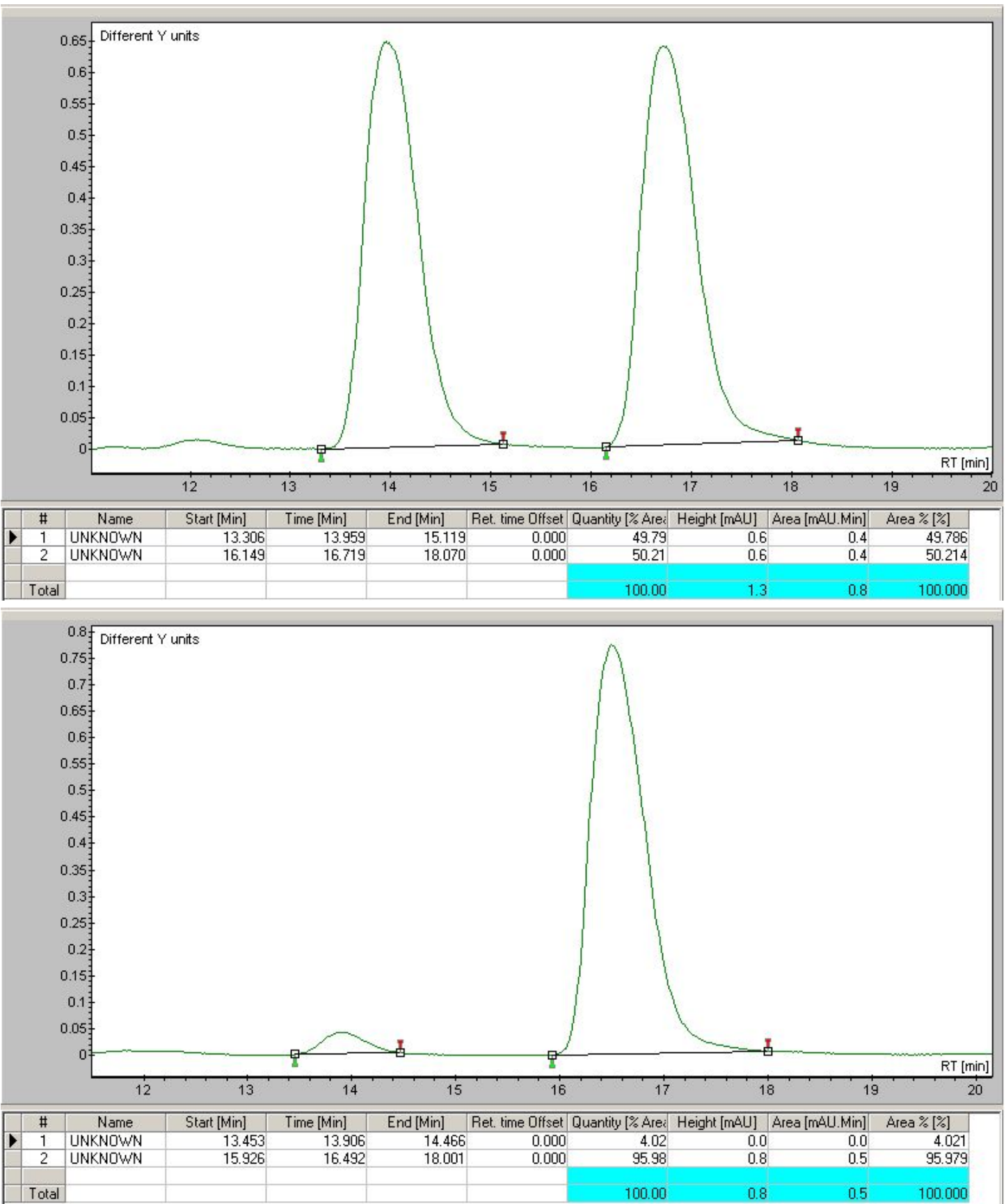
<smiles>COc1cccc([C@H](c2ccccc2C(F)(F)F)c2ccc3cc[nH]c3c2O)c1O</smiles>

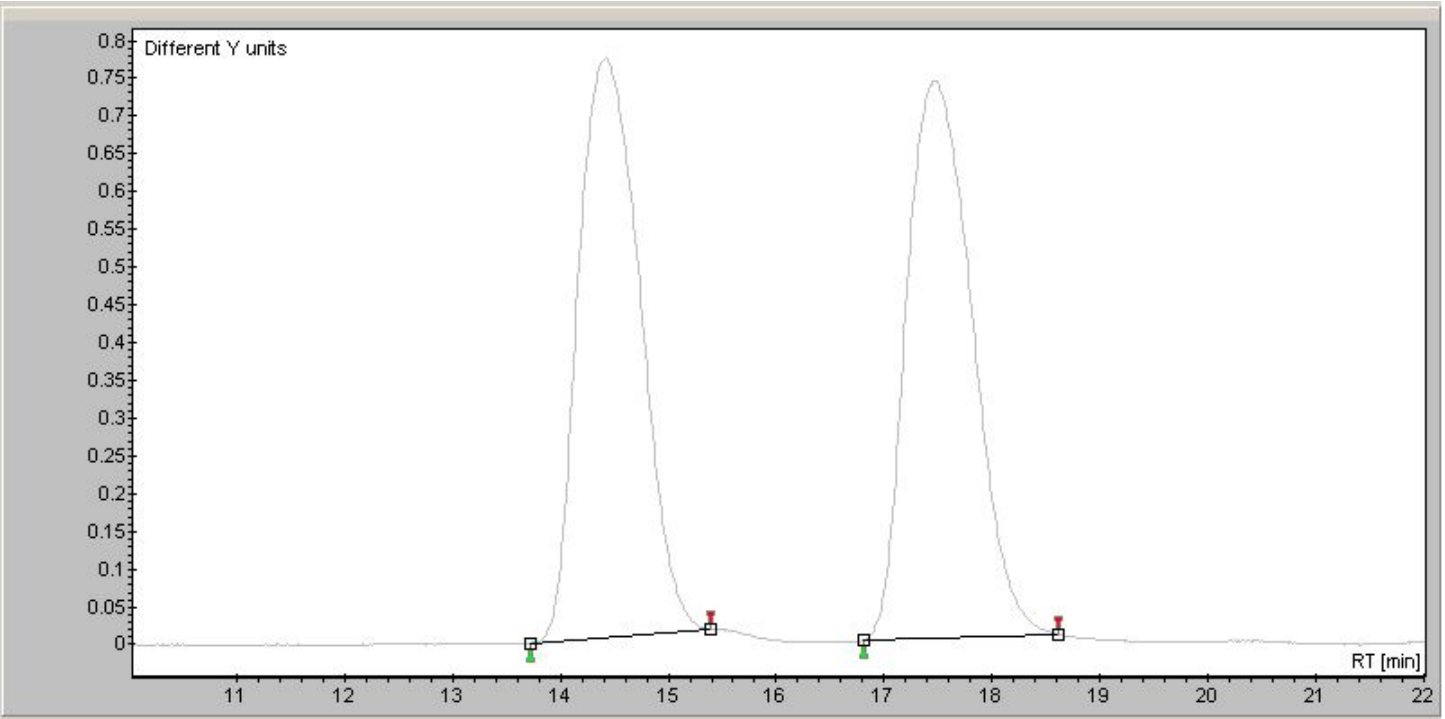

\begin{tabular}{|c|c|c|c|c|c|c|c|c|c|c|}
\hline & $\#$ & Name & Start [Min] & Time [Min] & End [Min] & Ret. time Offset & Quantity [\% Are: & Height [mAU] & Area [müU.Min] & Area \% [\%] \\
\hline 1 & 1 & UNKNOWN & 13.721 & 14.426 & 15.392 & 0.000 & 49.44 & 0.8 & 0.5 & 49.443 \\
\hline & 2 & UNKNOWN & 16.812 & 17.492 & 18.616 & 0.000 & 50.56 & 0.7 & 0.5 & 50.557 \\
\hline & Total & & & & & & 100.00 & 1.5 & 1.0 & 100.000 \\
\hline
\end{tabular}

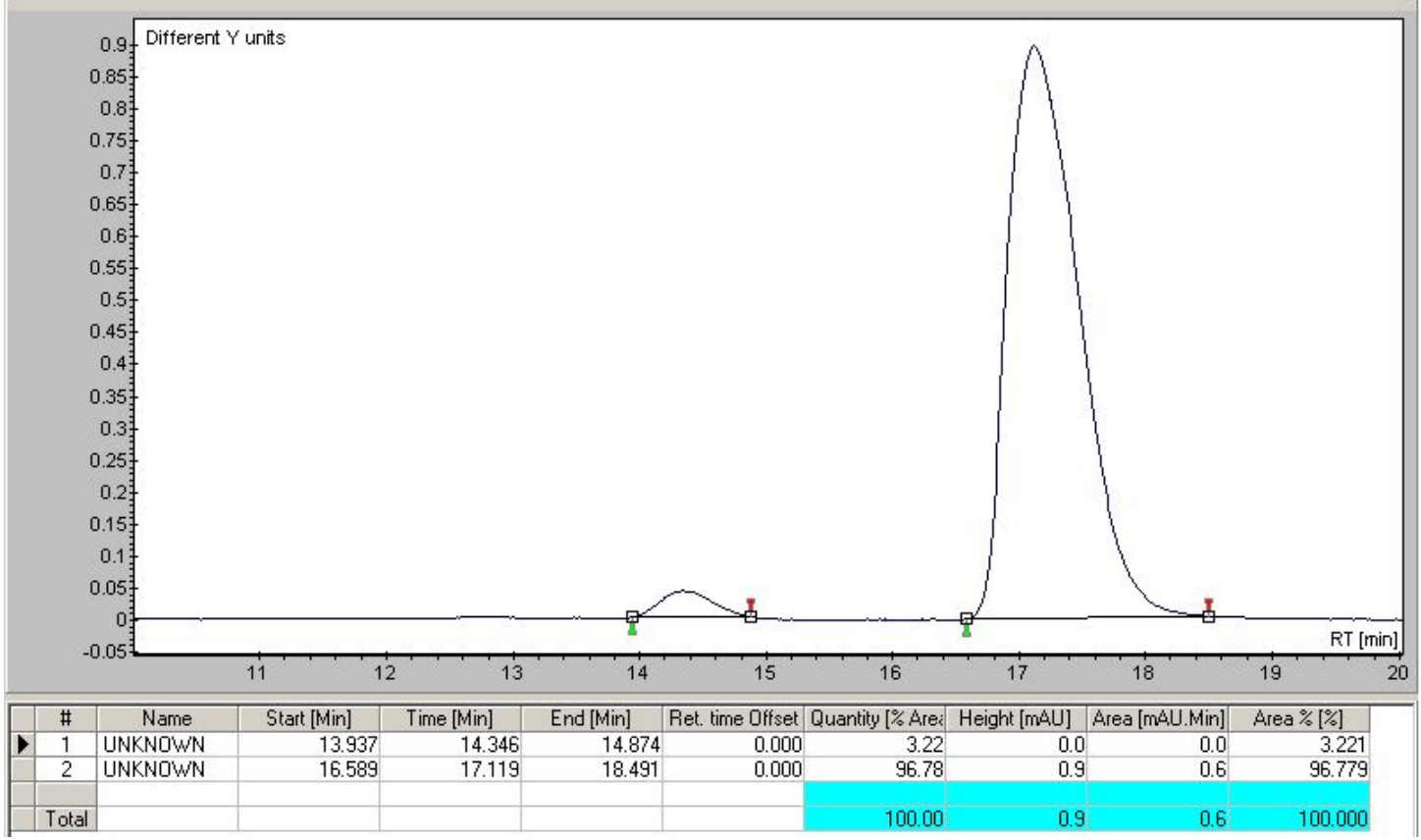


<smiles>COc1cccc([C@H](c2ccccc2)c2ccc3[nH]c4ccccc4c3c2O)c1O</smiles>
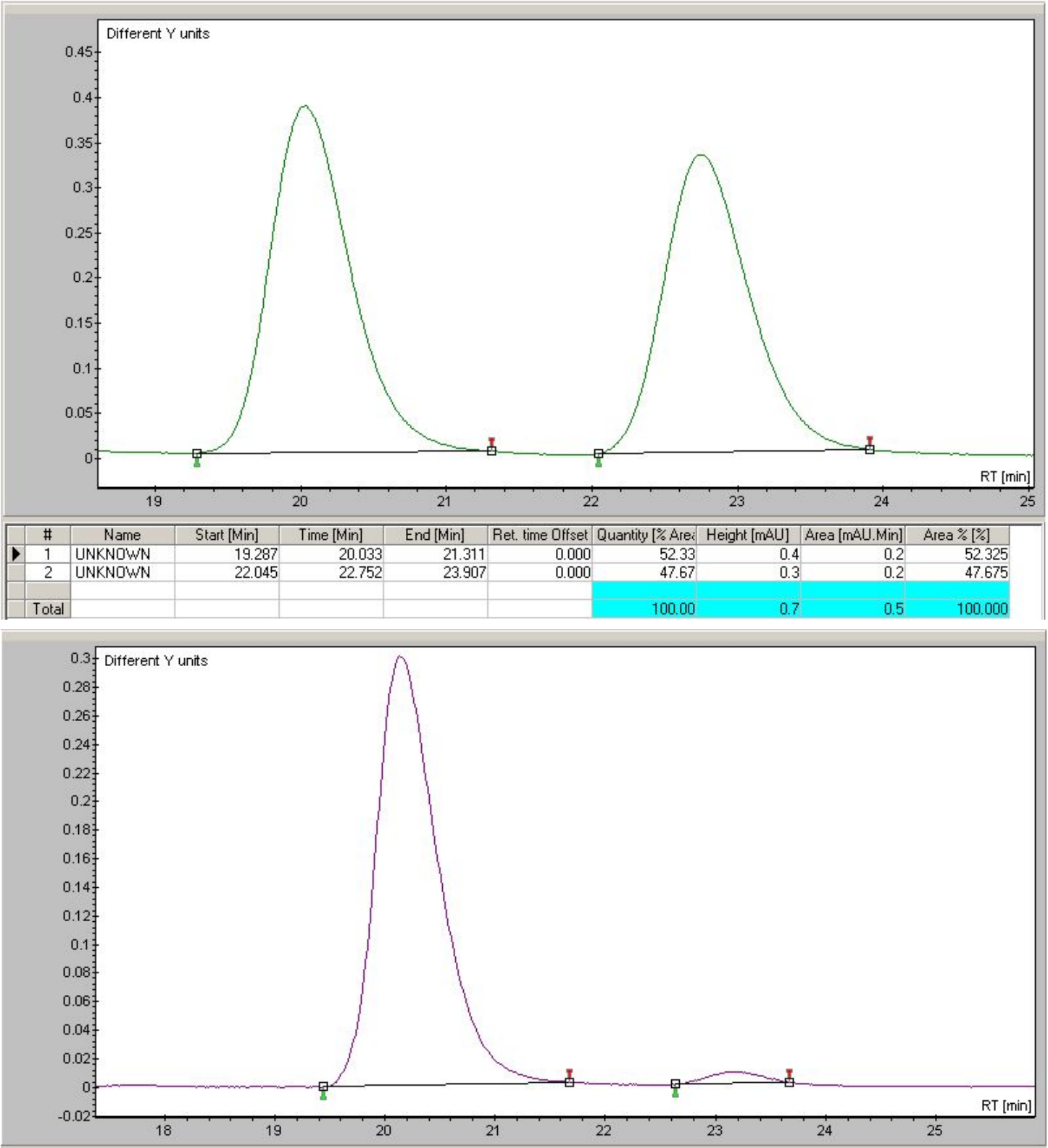

\begin{tabular}{|c|c|c|c|c|c|c|c|c|c|c|}
\hline & \# & Name & Start [Min] & Time [Min] & End [Min] & Ret. time Offset & Quantity [\% Are & Height [mBU] & Area [mAU.Min] & Area \% [\%] \\
\hline D & 1 & UNKNOWN & 19.442 & 20.132 & 21.674 & 0.000 & 97.81 & 0.3 & 0.2 & 97.811 \\
\hline & 2 & UNKNOWN & 22.636 & 23.158 & 23.669 & 0.000 & 2.19 & 0.0 & 0.0 & 2.189 \\
\hline & Total & & & & & & 100.00 & 0.3 & 0.2 & 100.000 \\
\hline
\end{tabular}<smiles>CCCOc1c(C(c2ccccc2)c2cccc(OC)c2[OH2+])ccc2[nH]ccc12</smiles> 


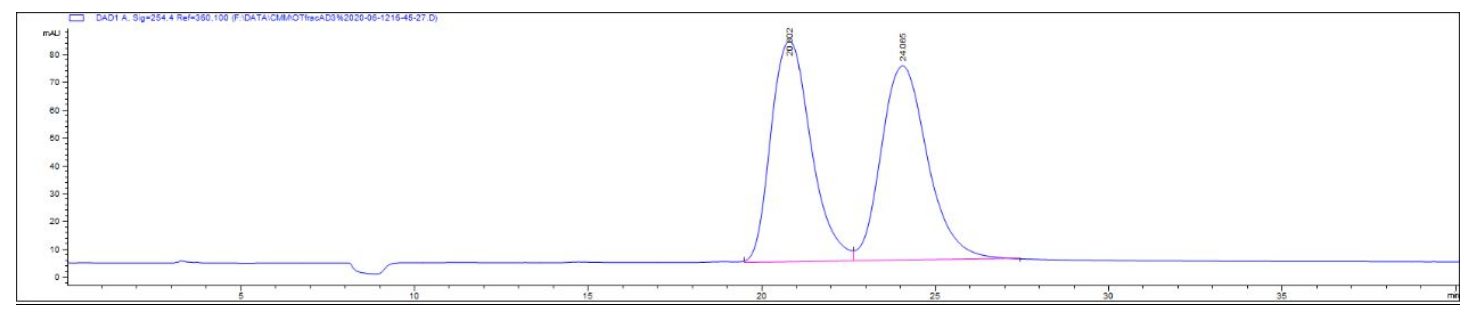

\begin{tabular}{|c|c|c|c|c|c|c|c|}
\hline$\#$ & 时间 & 峰面积 & 㠦高 & 崔赛 & 对称因子 & 崔面积 \% & 类型 \\
\hline 1 & 20.802 & 6399.5 & 79.4 & 1.3431 & 0 & 49.556 & MF \\
\hline 2 & 24.065 & 6514.2 & 70 & 1.5514 & 0.828 & 50.444 & FM \\
\hline
\end{tabular}

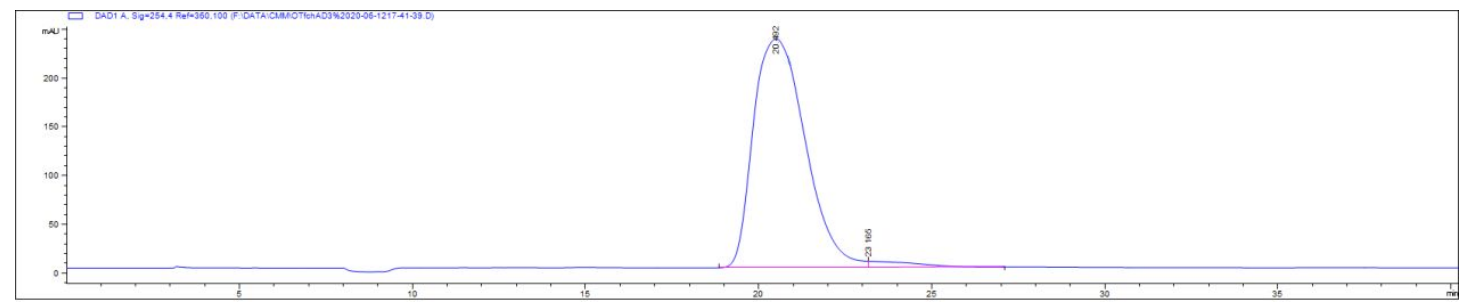

\begin{tabular}{|c|c|c|c|c|c|c|c|}
\hline$\#$ & 时间 & 峰面伿 & 崔高 & 峰赛 & 对称因子 & 峰面积 \% & 类型 \\
\hline 1 & 20.492 & 24464.6 & 235 & 1.7354 & 0.753 & 97.543 & MF \\
\hline 2 & 23.165 & 616.2 & 6.4 & 1.6139 & 0 & 2.457 & FM \\
\hline
\end{tabular}

\section{2. ${ }^{1} \mathrm{H}-\mathrm{NMR}$ and ${ }^{13} \mathrm{C}-\mathrm{NMR}$ spectra}<smiles>COc1cccc(C(F)c2ccccc2)c1O</smiles>

1a

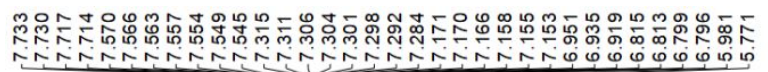

${ }^{1} \mathrm{H}$ NMR $\left(500 \mathrm{MHz}, \mathrm{CDCl}_{3}\right)$

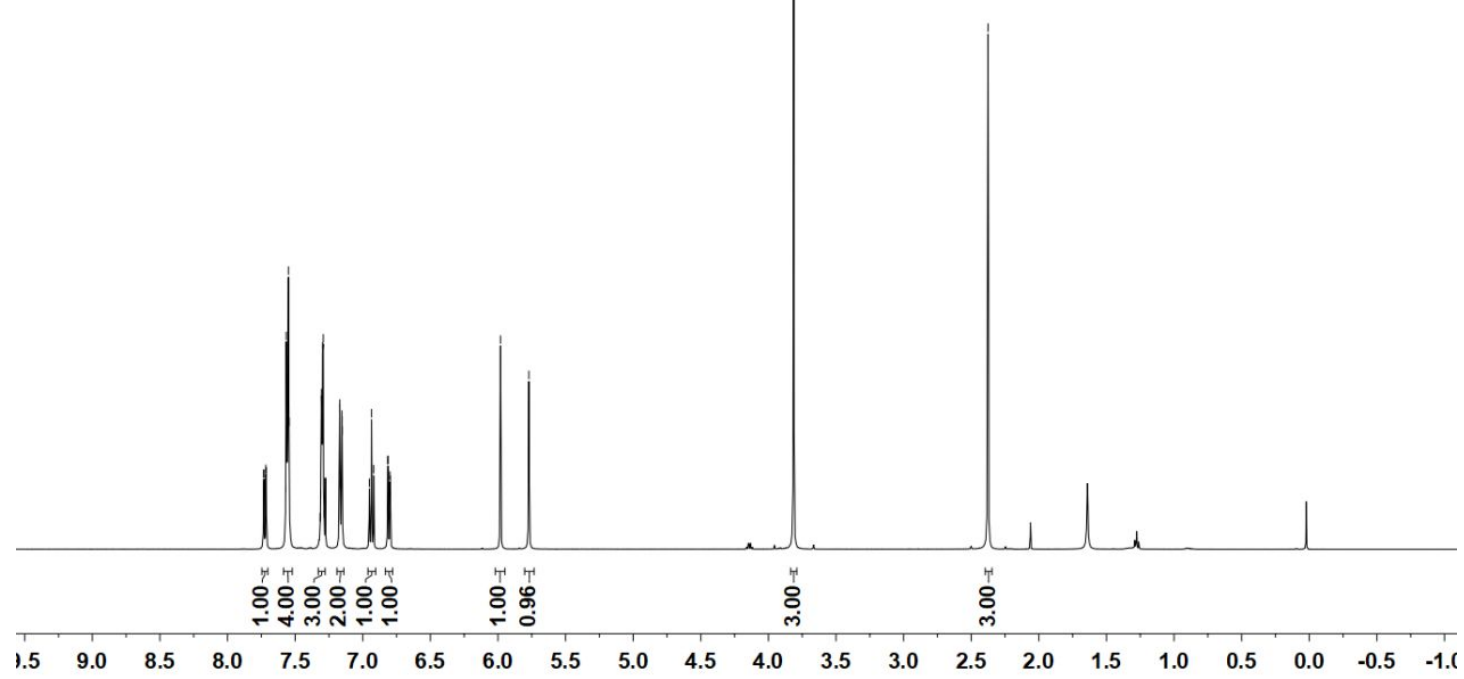




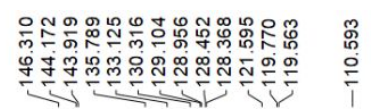

${ }^{13} \mathrm{C}\left\{{ }^{1} \mathrm{H}\right\}$ NMR $\left(126 \mathrm{MHz}, \mathrm{CDCl}_{3}\right)$

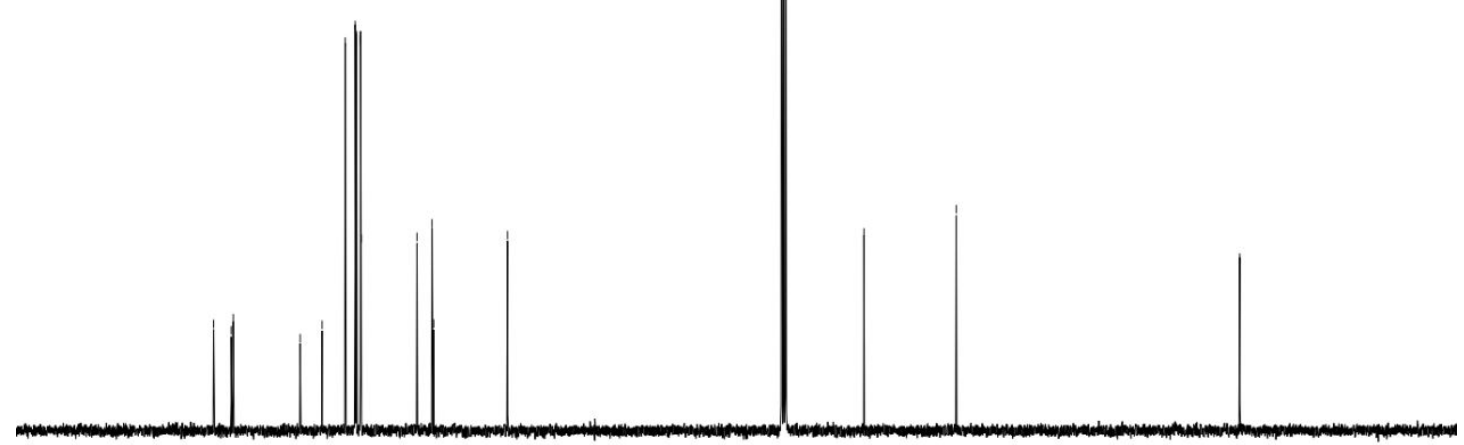

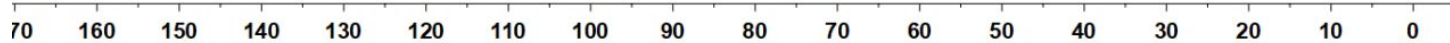<smiles>CCOc1cccc(C(C)c2ccccc2)c1O</smiles>

$1 \mathrm{~b}$

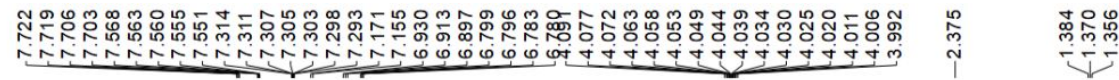

${ }^{1} \mathrm{H}$ NMR (500 MHz, $\left.\mathrm{CDCl}_{3}\right)$

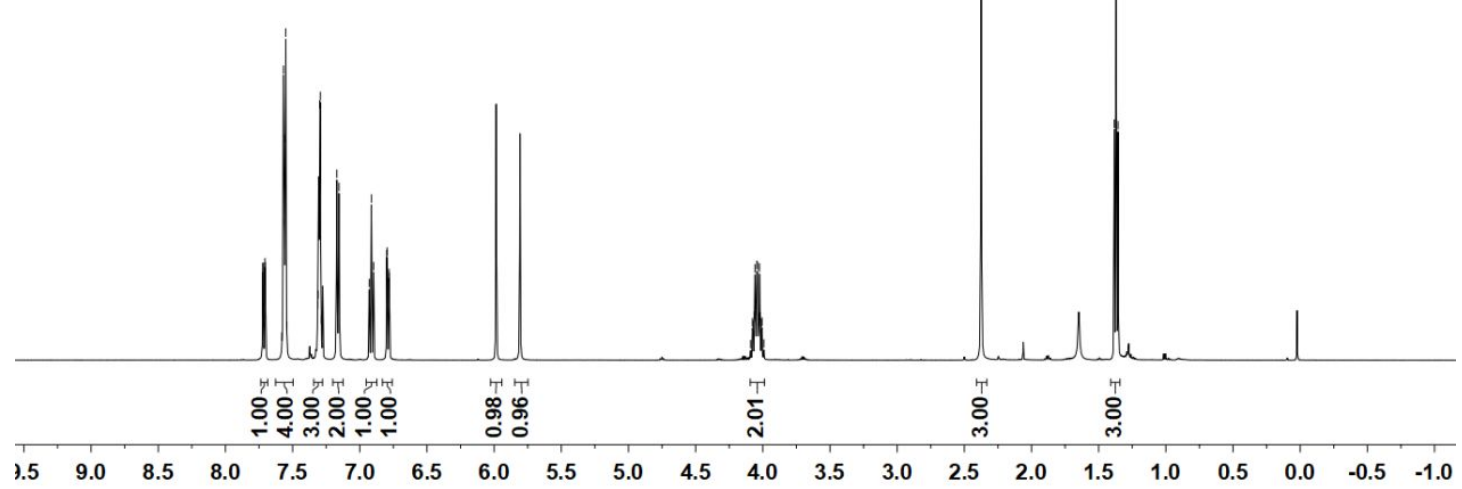


${ }^{13} \mathrm{C}\left\{{ }^{1} \mathrm{H}\right\}$ NMR $\left(126 \mathrm{MHz}, \mathrm{CDCl}_{3}\right)$
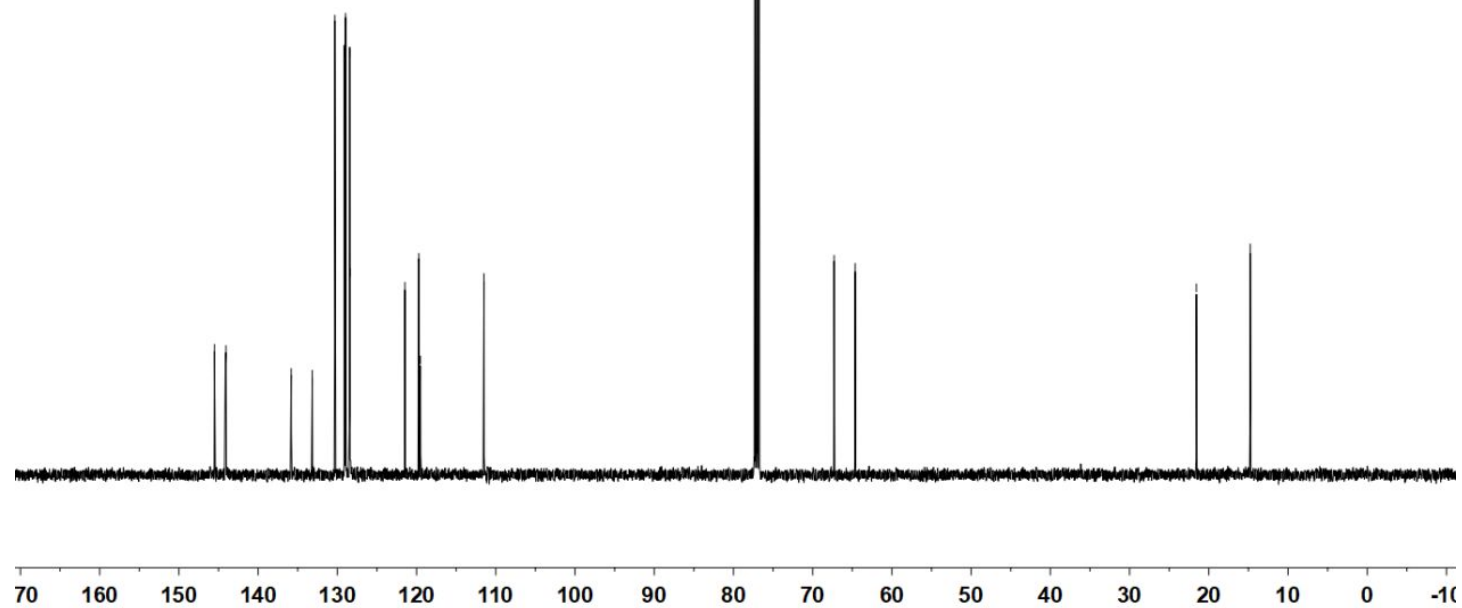<smiles>COc1ccc(C(F)c2ccccc2)c(O)c1</smiles>

1c

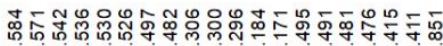

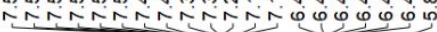

${ }^{1} \mathrm{H}$ NMR (600 MHz, $\mathrm{CDCl}_{3}$ )

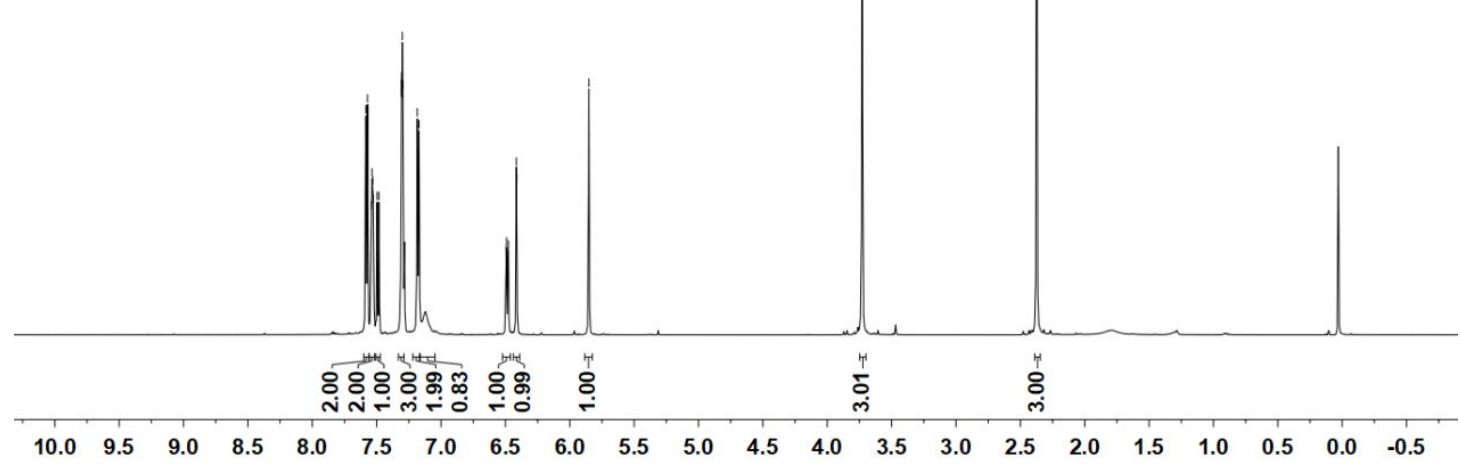


${ }^{13} \mathrm{C}\left\{{ }^{1} \mathrm{H}\right\}$ NMR $\left(151 \mathrm{MHz}, \mathrm{CDCl}_{3}\right)$

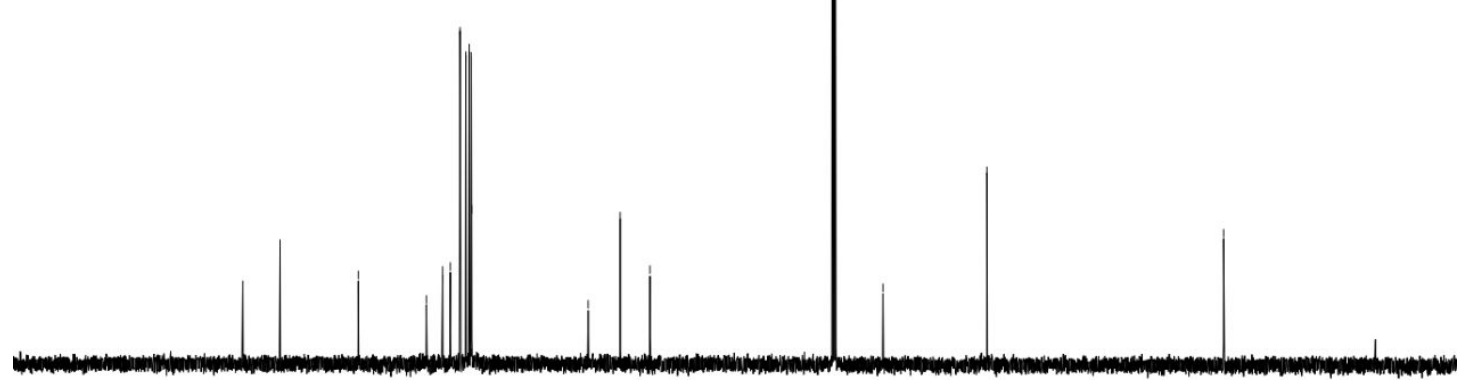

$\begin{array}{lllllllllllllllllllll}190 & 180 & 170 & 160 & 150 & 140 & 130 & 120 & 110 & 100 & 90 & 80 & 70 & 60 & 50 & 40 & 30 & 20 & 10 & 0 & -16\end{array}$<smiles>Oc1cc(Br)cc(C(P)c2ccccc2)c1O</smiles>

1d

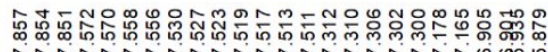

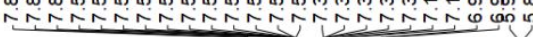

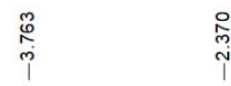

${ }^{1} \mathrm{H}$ NMR (600 MHz, $\left.\mathrm{CDCl}_{3}\right)$

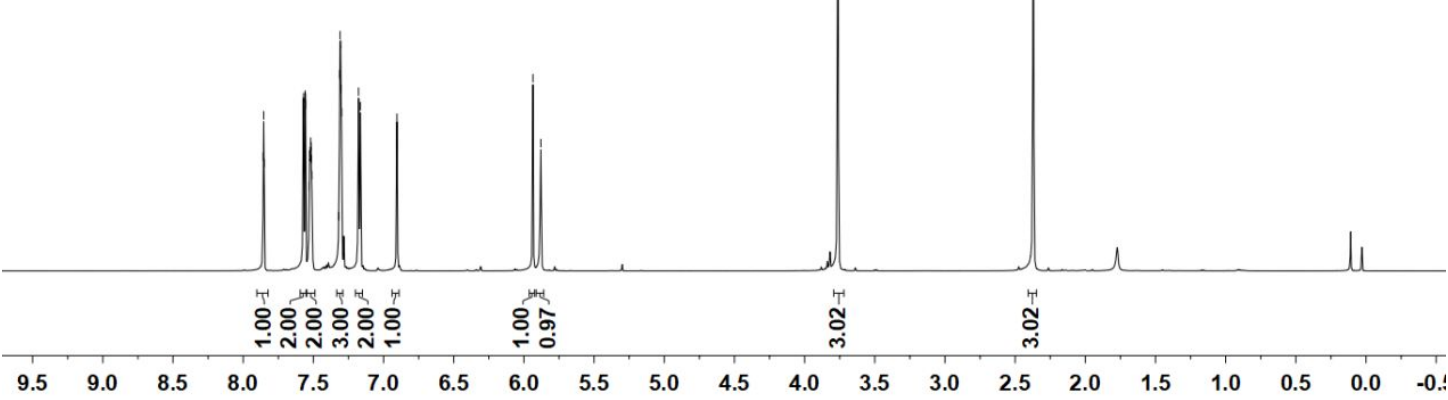


ร응

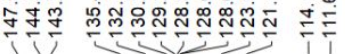

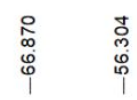

$\stackrel{\frac{0}{0}}{\stackrel{\oplus}{\sim}}$

${ }^{13} \mathrm{C}\left\{{ }^{1} \mathrm{H}\right\} \mathrm{NMR}\left(151 \mathrm{MHz}, \mathrm{CDCl}_{3}\right)$

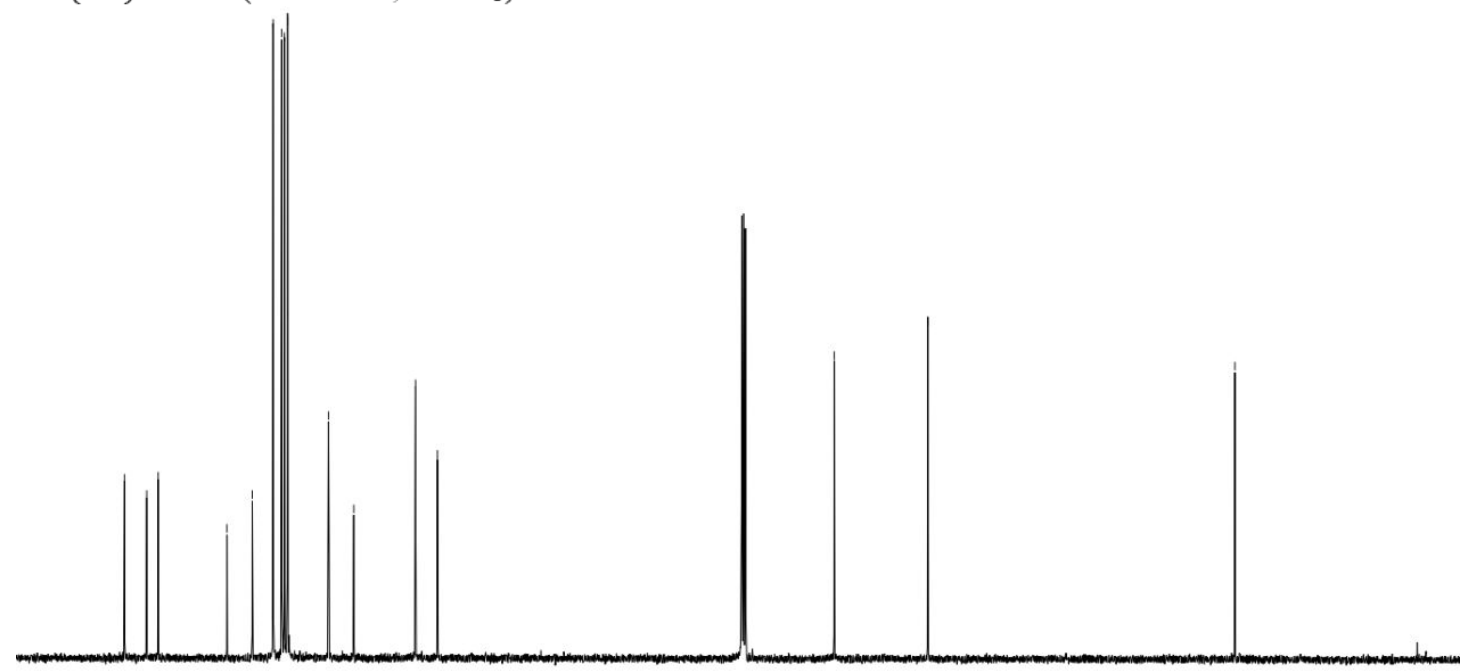

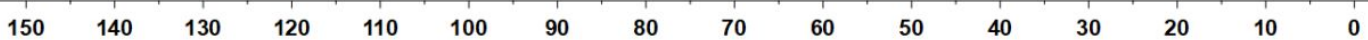<smiles>Oc1ccccc1C(P)c1ccccc1</smiles>

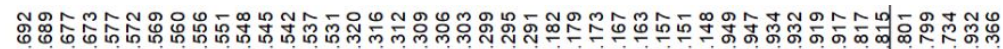

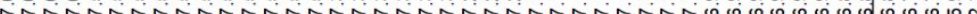

${ }^{1} \mathrm{H}$ NMR $\left(500 \mathrm{MHz}, \mathrm{CDCl}_{3}\right)$

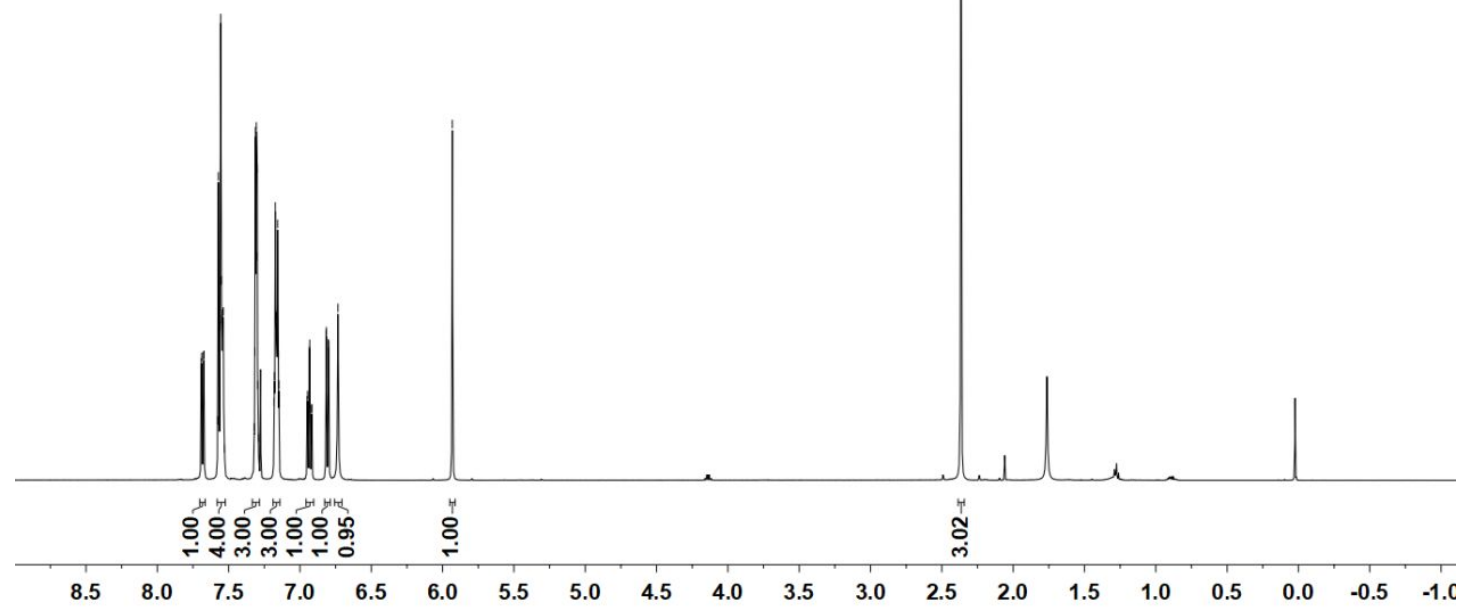


${ }^{13} \mathrm{C}\left\{{ }^{1} \mathrm{H}\right\} \mathrm{NMR}\left(126 \mathrm{MHz}, \mathrm{CDCl}_{3}\right)$
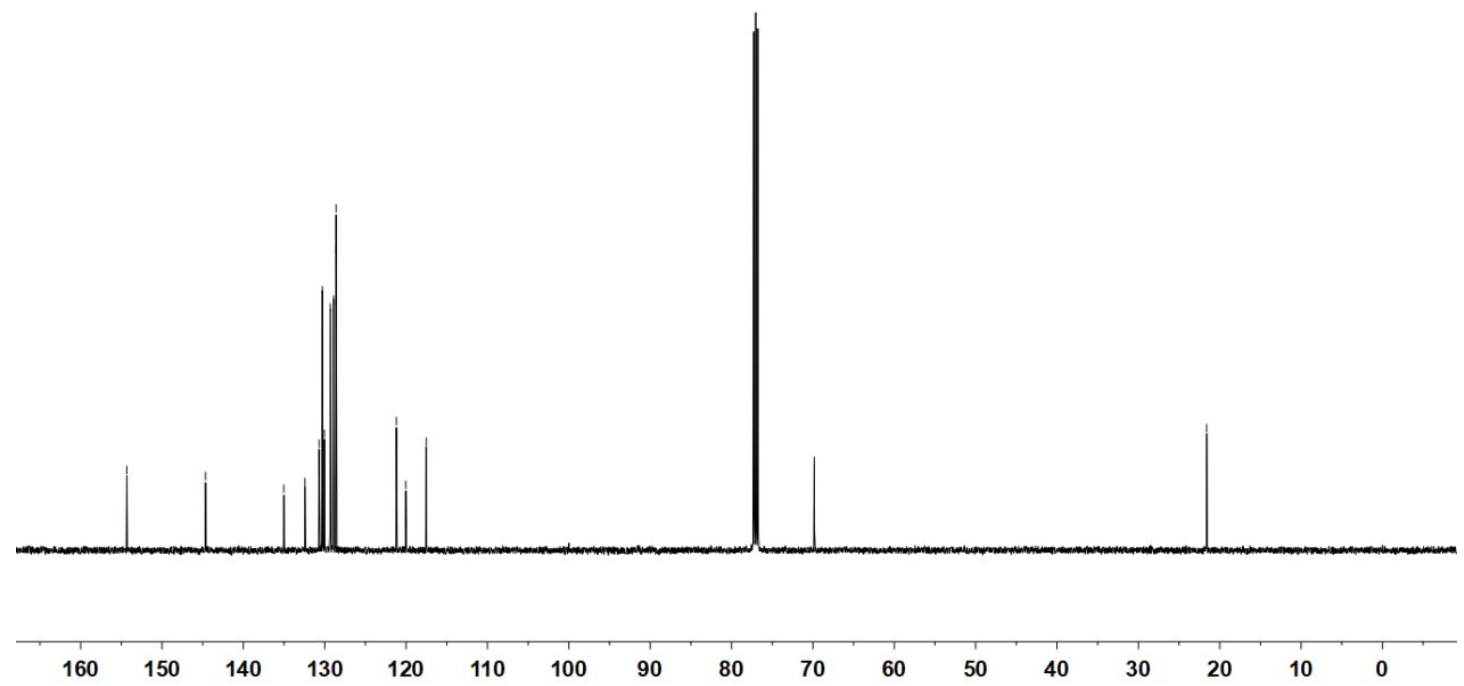<smiles>COc1cccc(C([18OH])c2cccc(C)c2)c1O</smiles>

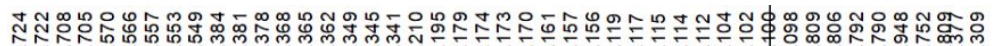

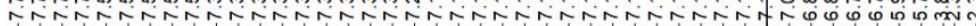

${ }^{1} \mathrm{H}$ NMR $\left(500 \mathrm{MHz}, \mathrm{CDCl}_{3}\right)$

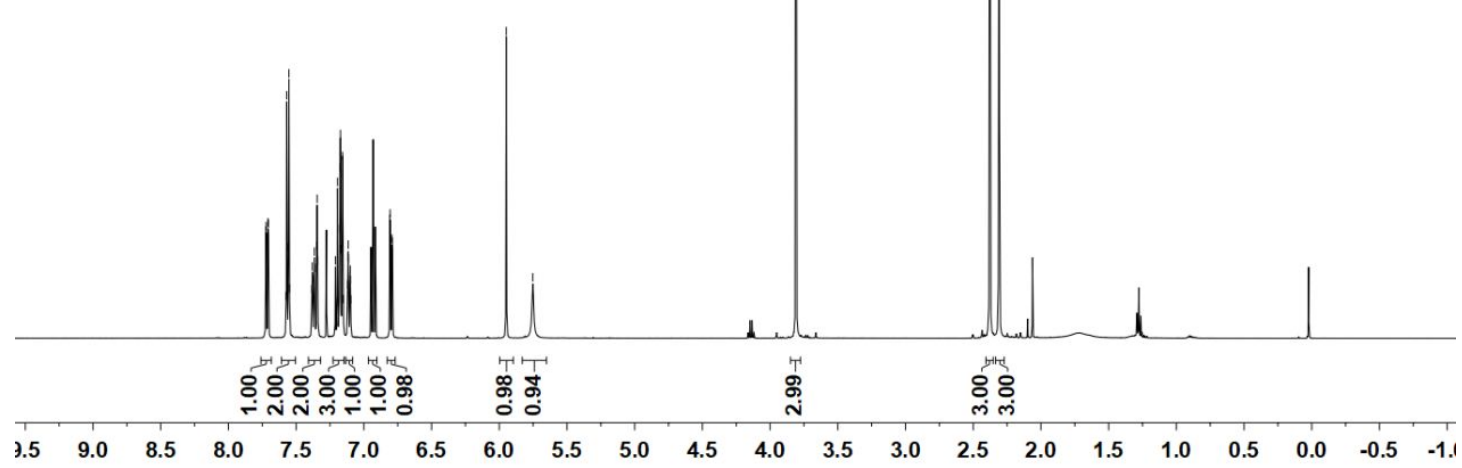



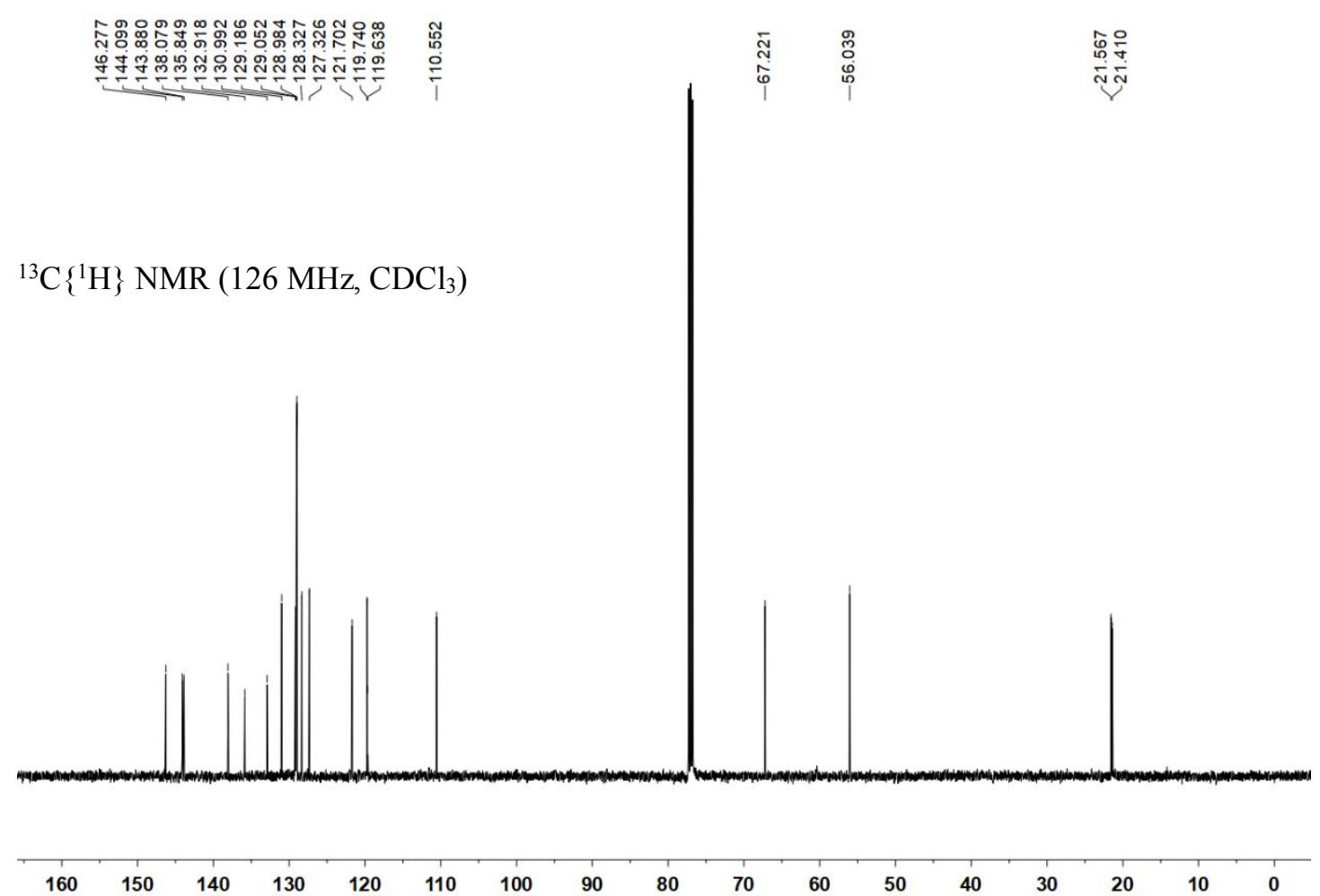<smiles>COc1cccc(C([125I])c2ccc(C)cc2)c1O</smiles>

19

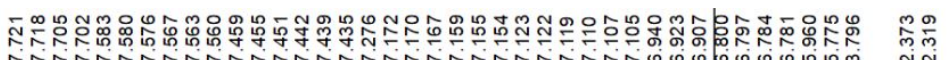

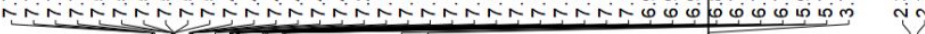

${ }^{1} \mathrm{H}$ NMR $\left(500 \mathrm{MHz}, \mathrm{CDCl}_{3}\right)$

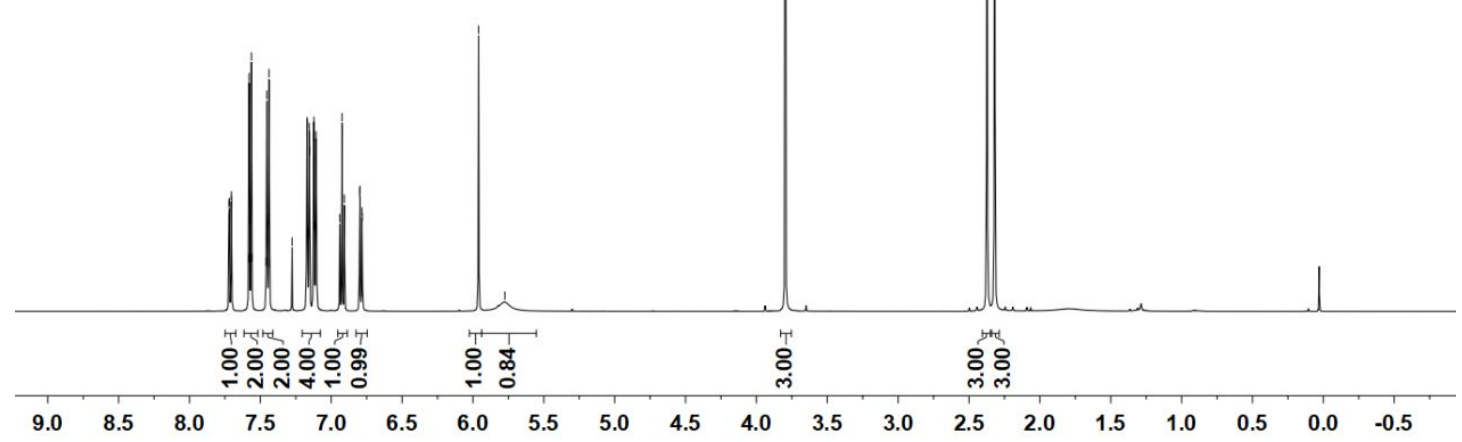




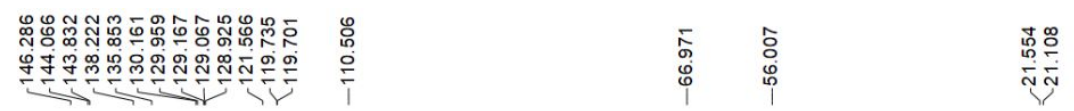

${ }^{13} \mathrm{C}\left\{{ }^{1} \mathrm{H}\right\}$ NMR $\left(126 \mathrm{MHz}, \mathrm{CDCl}_{3}\right)$

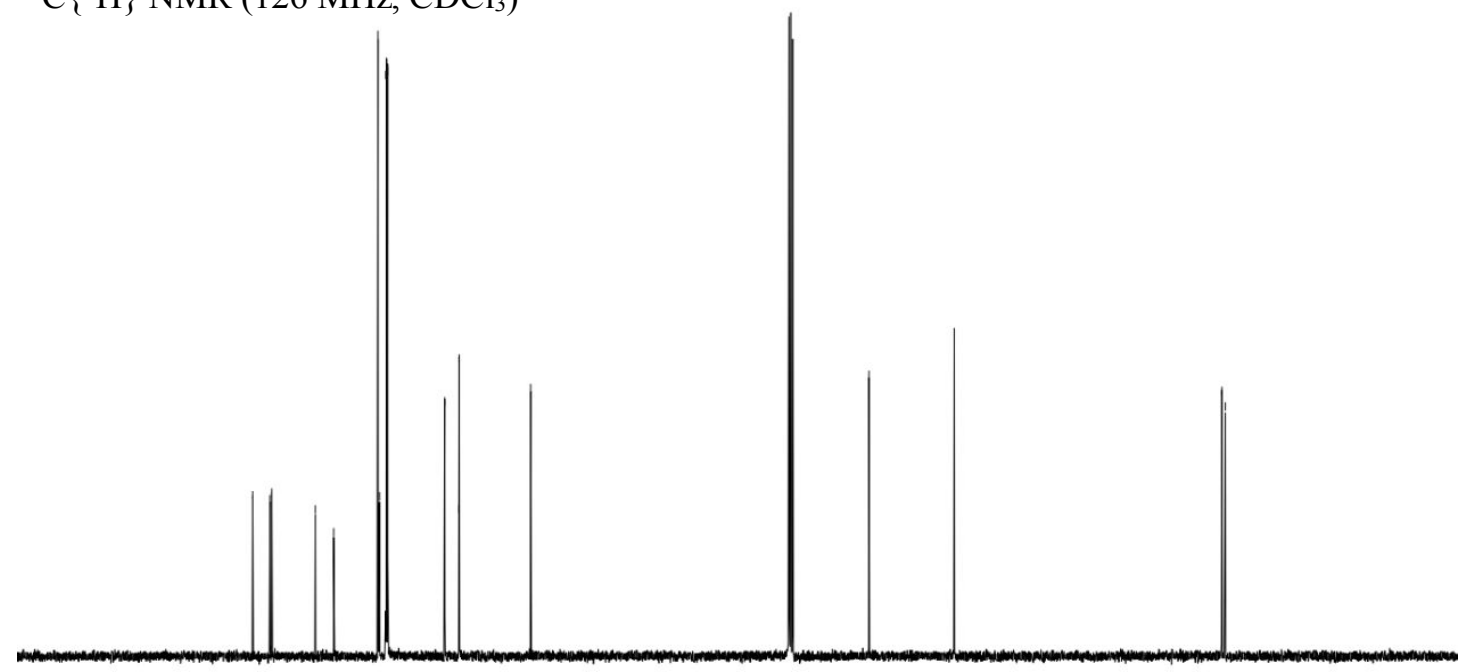

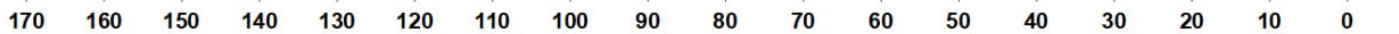<smiles>COc1cccc(C([13CH3])c2cccc(OC)c2O)c1</smiles>

$1 \mathrm{~h}$

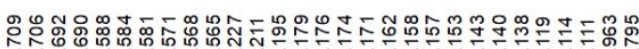

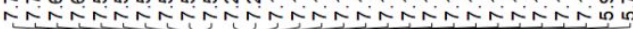

${ }^{1} \mathrm{H}$ NMR $\left(500 \mathrm{MHz}, \mathrm{CDCl}_{3}\right)$

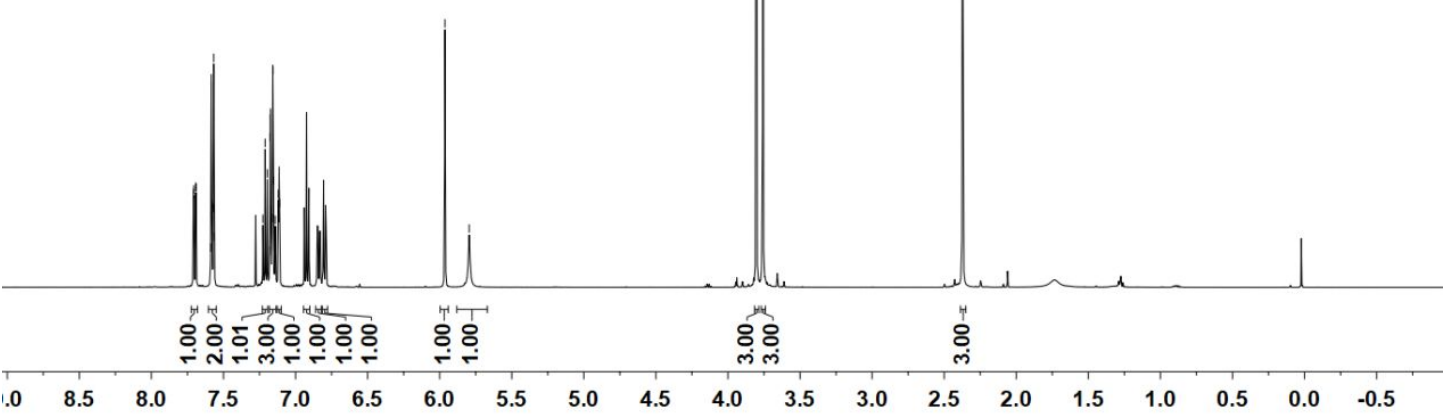


${ }^{13} \mathrm{C}\left\{{ }^{1} \mathrm{H}\right\}$ NMR $\left(126 \mathrm{MHz}, \mathrm{CDCl}_{3}\right)$

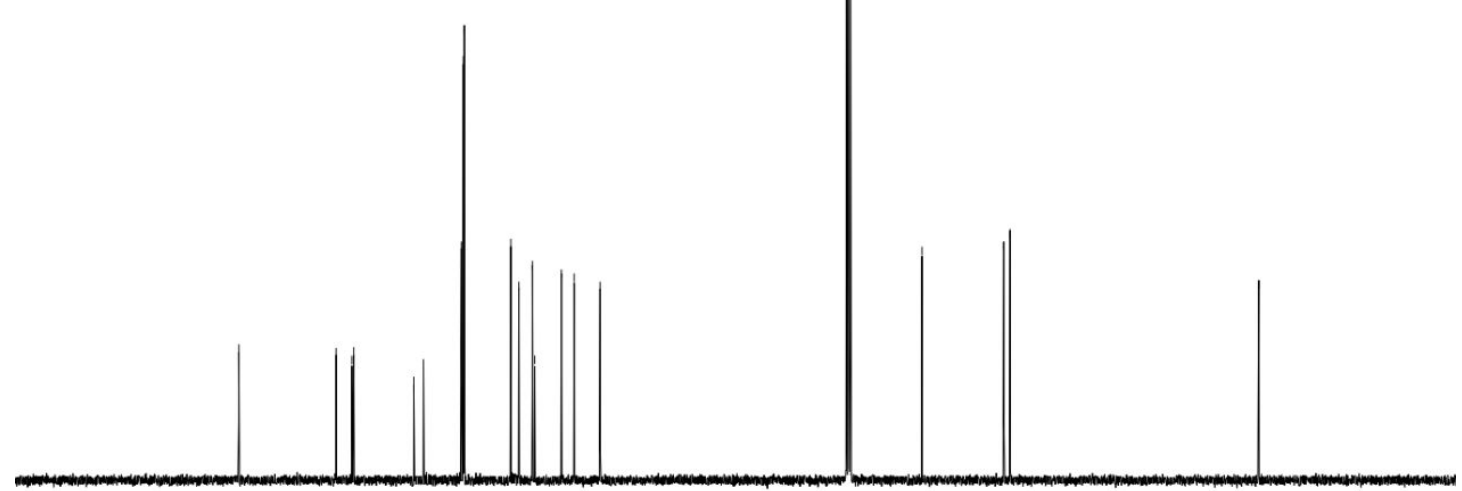

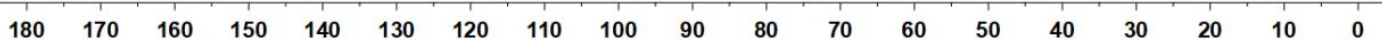<smiles>COc1cccc(C([18OH])c2cccc(OC)c2O)c1</smiles>

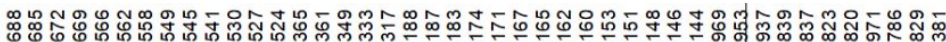

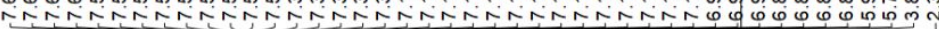

${ }^{1} \mathrm{H} \mathrm{NMR}\left(500 \mathrm{MHz}, \mathrm{CDCl}_{3}\right)$

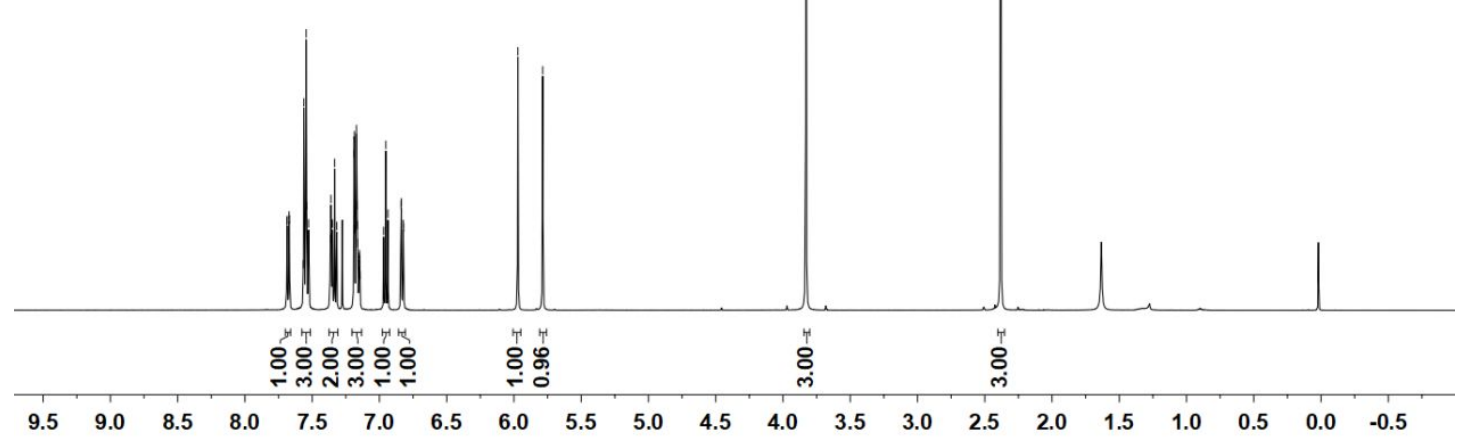


${ }^{13} \mathrm{C}\left\{{ }^{1} \mathrm{H}\right\}$ NMR $\left(126 \mathrm{MHz}, \mathrm{CDCl}_{3}\right)$
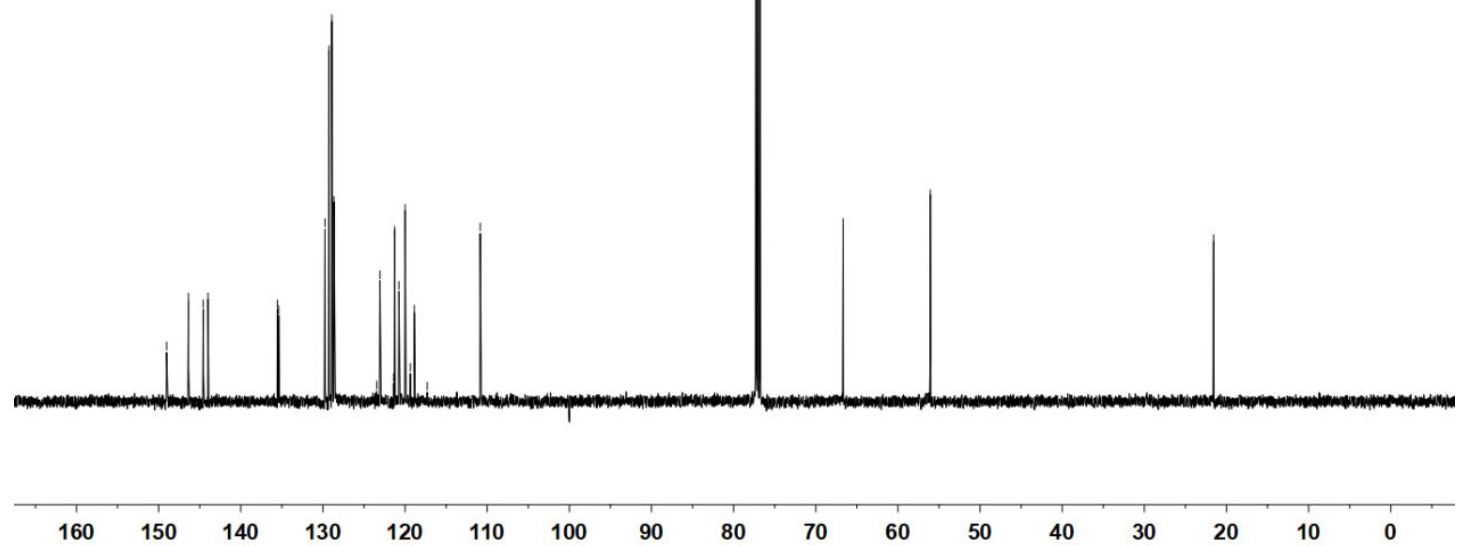<smiles>COc1ccc(C([123I])c2cccc(OC(F)(F)F)c2O)cc1</smiles>

$1 \mathrm{j}$

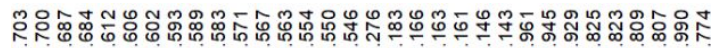

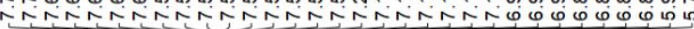

${ }^{1} \mathrm{H}$ NMR (500 MHz, $\left.\mathrm{CDCl}_{3}\right)$

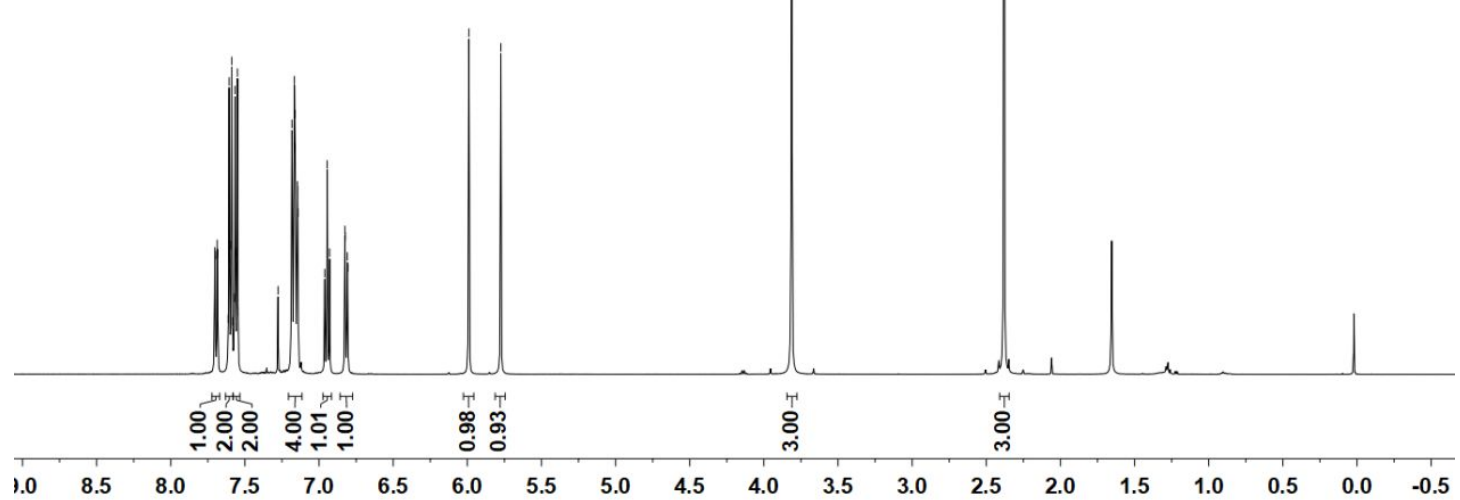


${ }^{13} \mathrm{C}\left\{{ }^{1} \mathrm{H}\right\} \mathrm{NMR}\left(126 \mathrm{MHz}, \mathrm{CDCl}_{3}\right)$
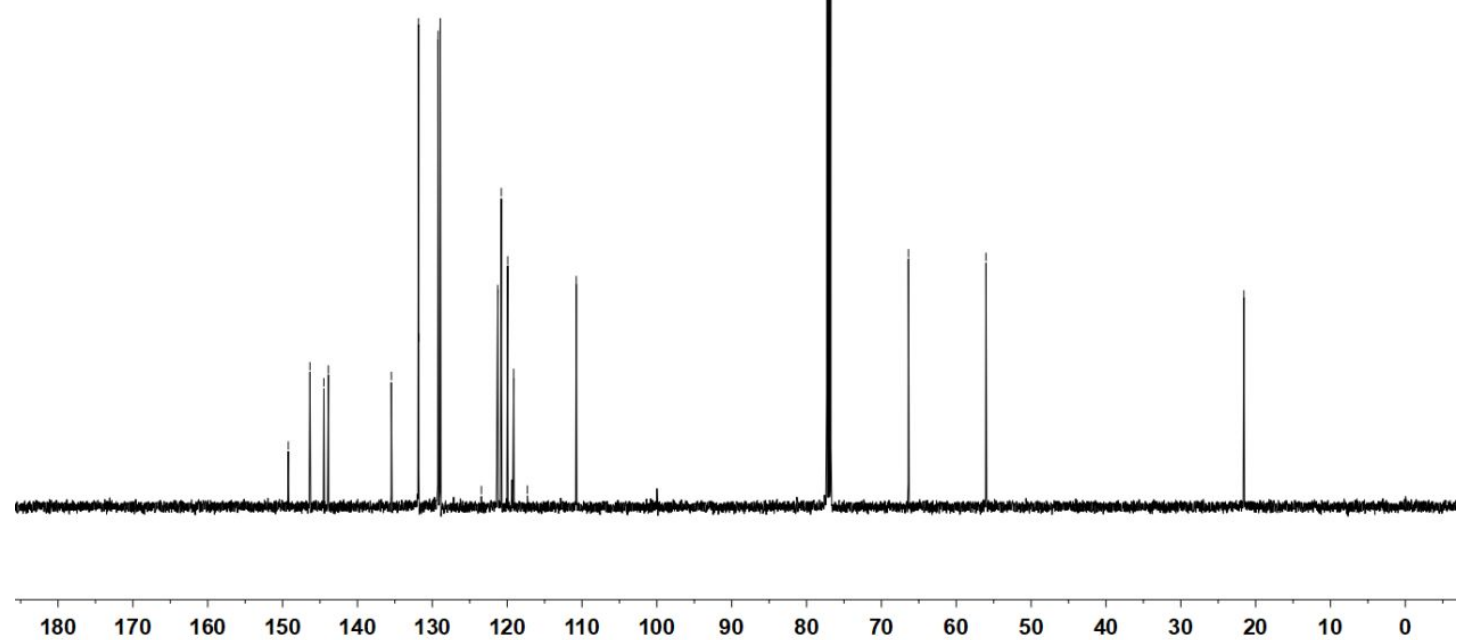<smiles>COc1cccc(C([18OH])c2ccc(F)cc2)c1O</smiles>

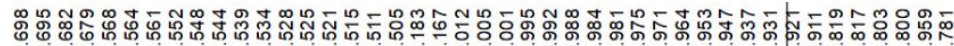

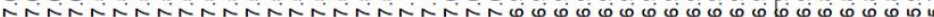

${ }^{1} \mathrm{H} \mathrm{NMR}\left(500 \mathrm{MHz}, \mathrm{CDCl}_{3}\right)$

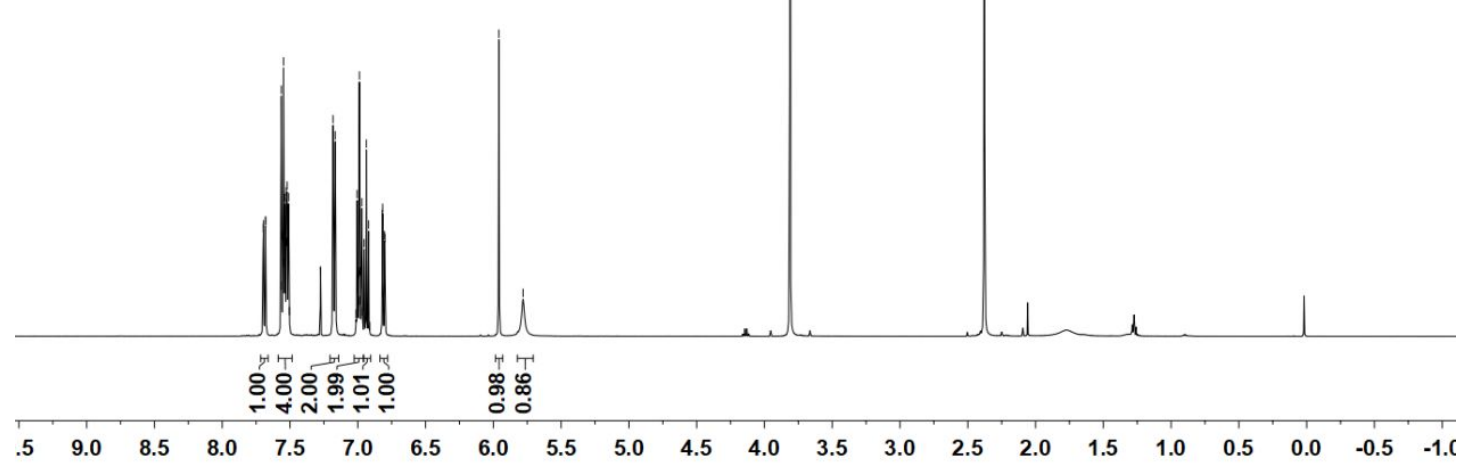



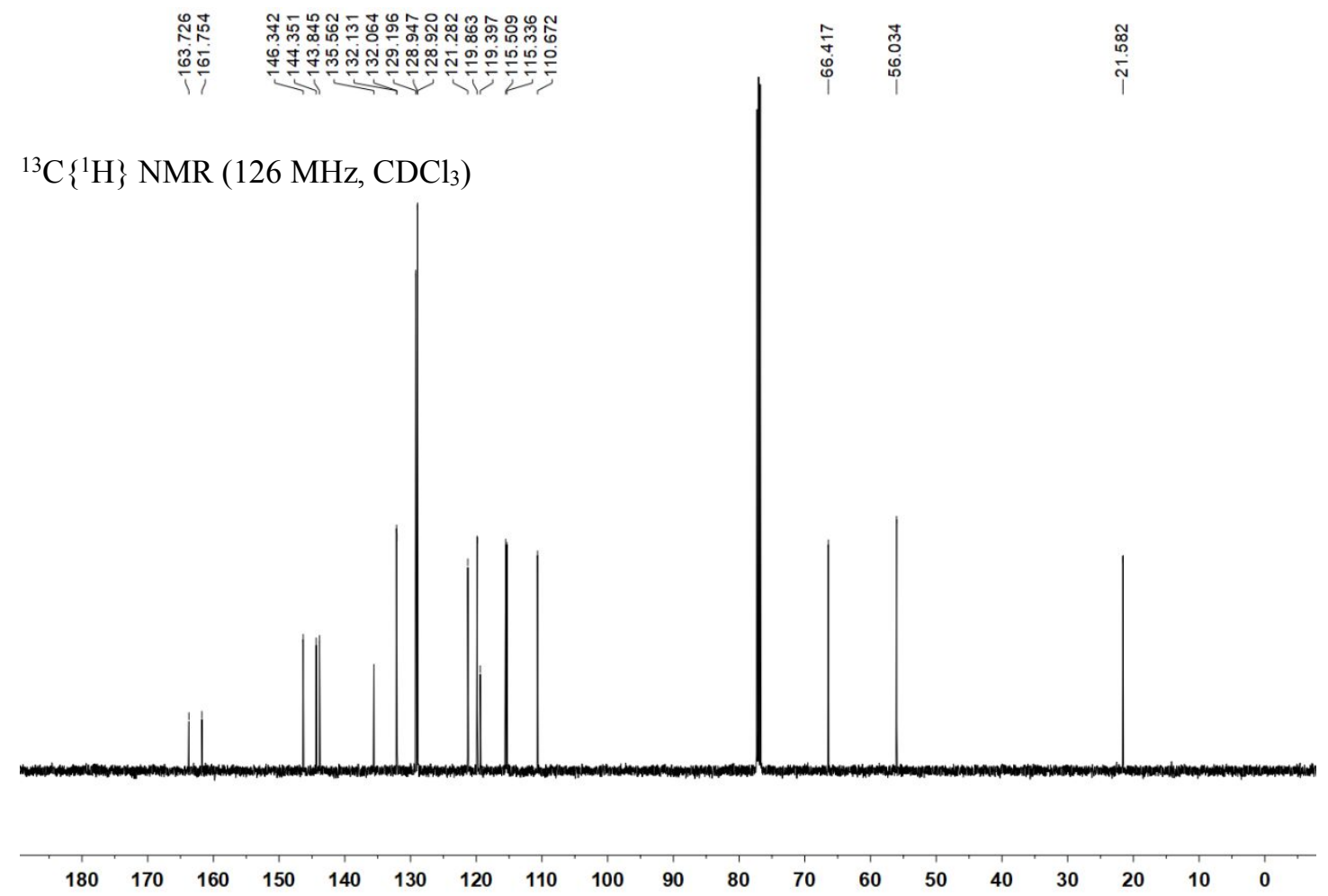<smiles>Oc1cccc(C([18OH])c2ccc(Cl)cc2)c1O</smiles>

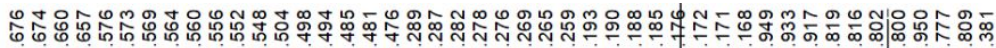

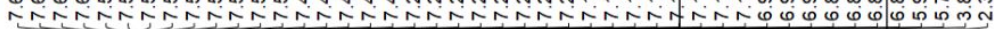

${ }^{1} \mathrm{H}$ NMR $\left(500 \mathrm{MHz}, \mathrm{CDCl}_{3}\right)$

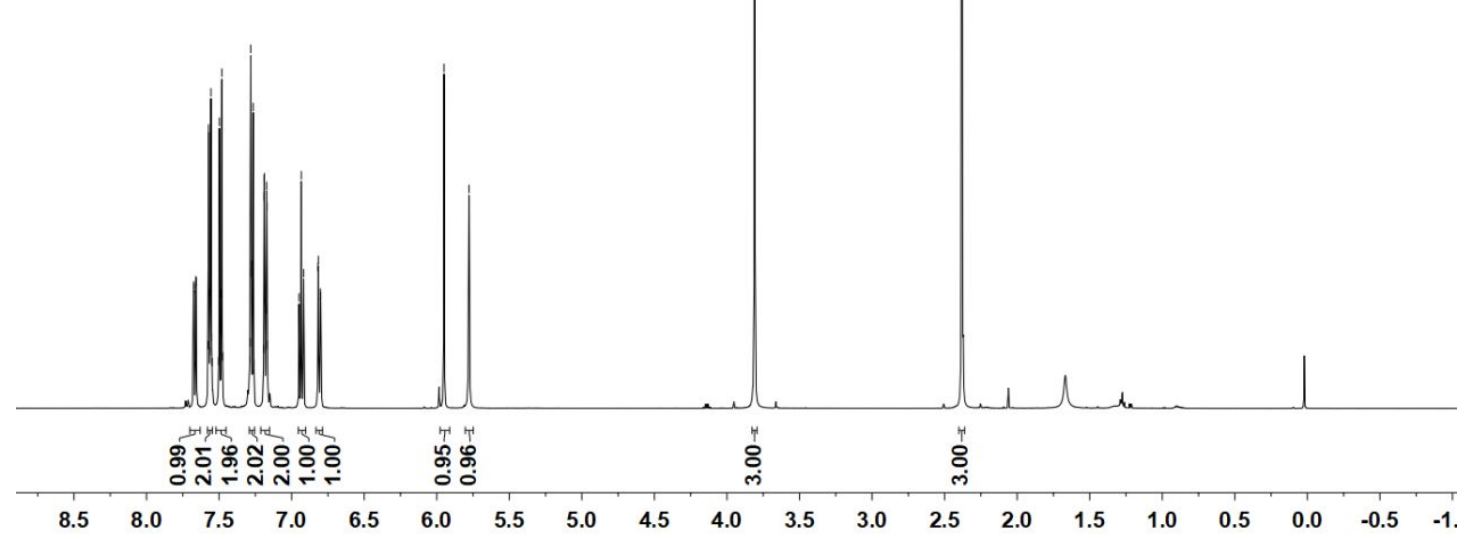


${ }^{13} \mathrm{C}\left\{{ }^{1} \mathrm{H}\right\}$ NMR $\left(126 \mathrm{MHz}, \mathrm{CDCl}_{3}\right)$

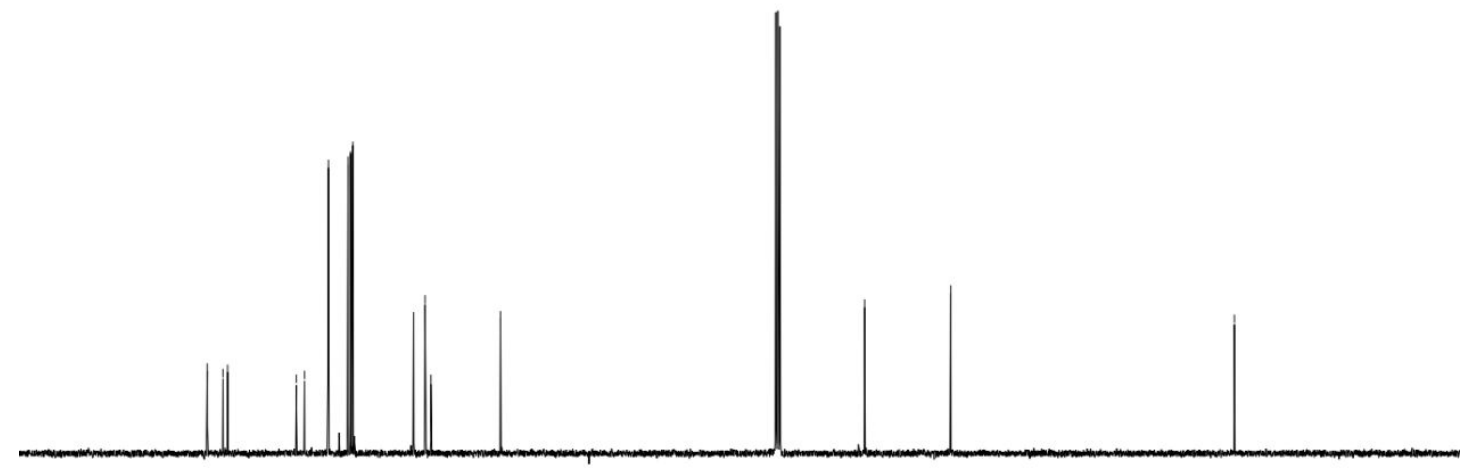

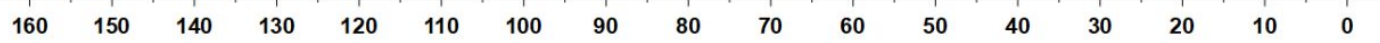<smiles>Oc1cccc(C([18F])c2ccccc2C(F)(F)F)c1O</smiles>

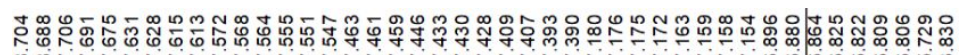

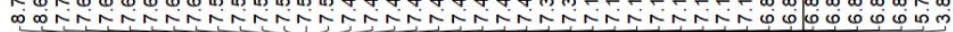

${ }^{1} \mathrm{H} \mathrm{NMR}\left(500 \mathrm{MHz}, \mathrm{CDCl}_{3}\right)$

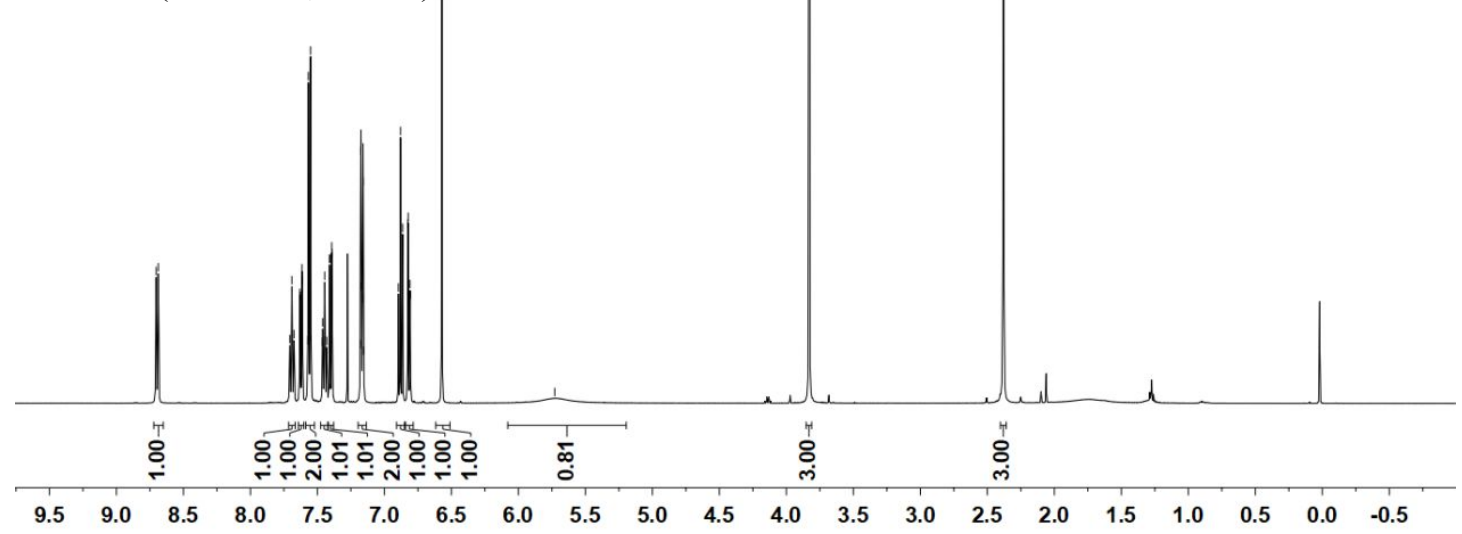


${ }^{13} \mathrm{C}\left\{{ }^{1} \mathrm{H}\right\}$ NMR $\left(126 \mathrm{MHz}, \mathrm{CDCl}_{3}\right)$

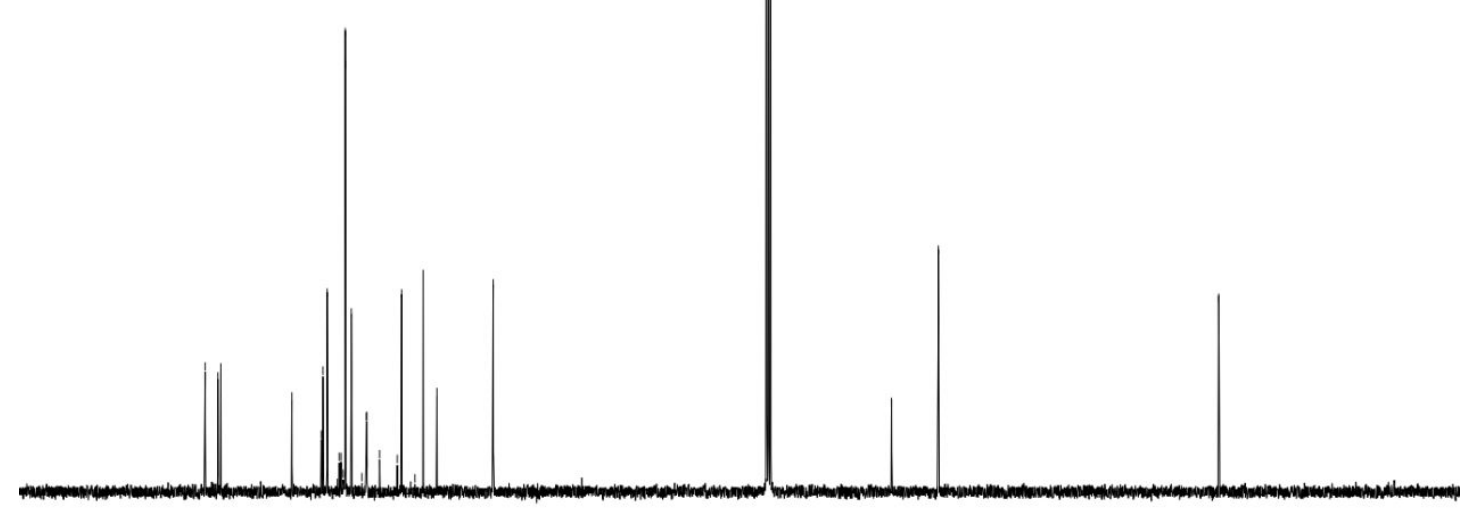

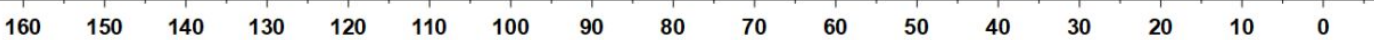<smiles>Oc1cccc(C([18F])c2cccc(C(F)(F)F)c2)c1O</smiles>

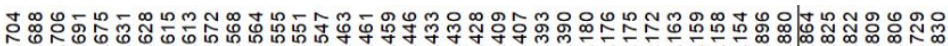

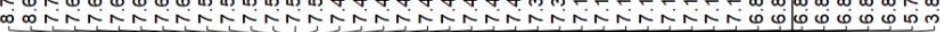

${ }^{1} \mathrm{H}$ NMR $\left(500 \mathrm{MHz}, \mathrm{CDCl}_{3}\right)$

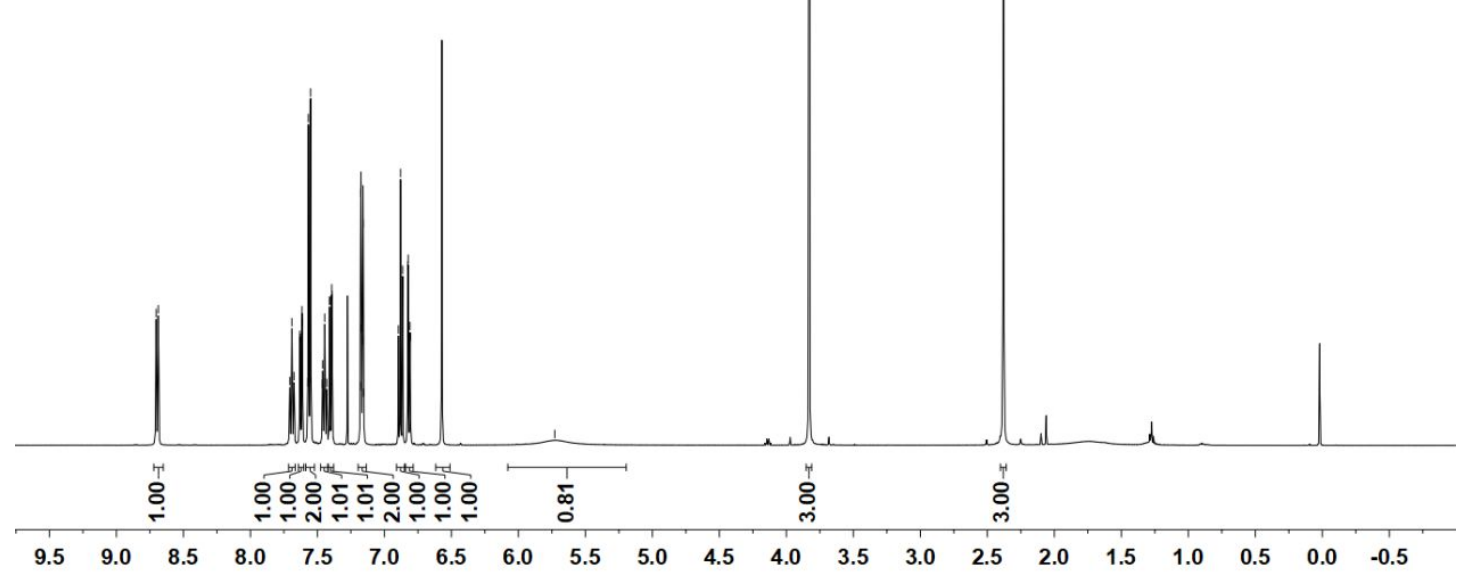


${ }^{13} \mathrm{C}\left\{{ }^{1} \mathrm{H}\right\}$ NMR $\left(126 \mathrm{MHz}, \mathrm{CDCl}_{3}\right)$
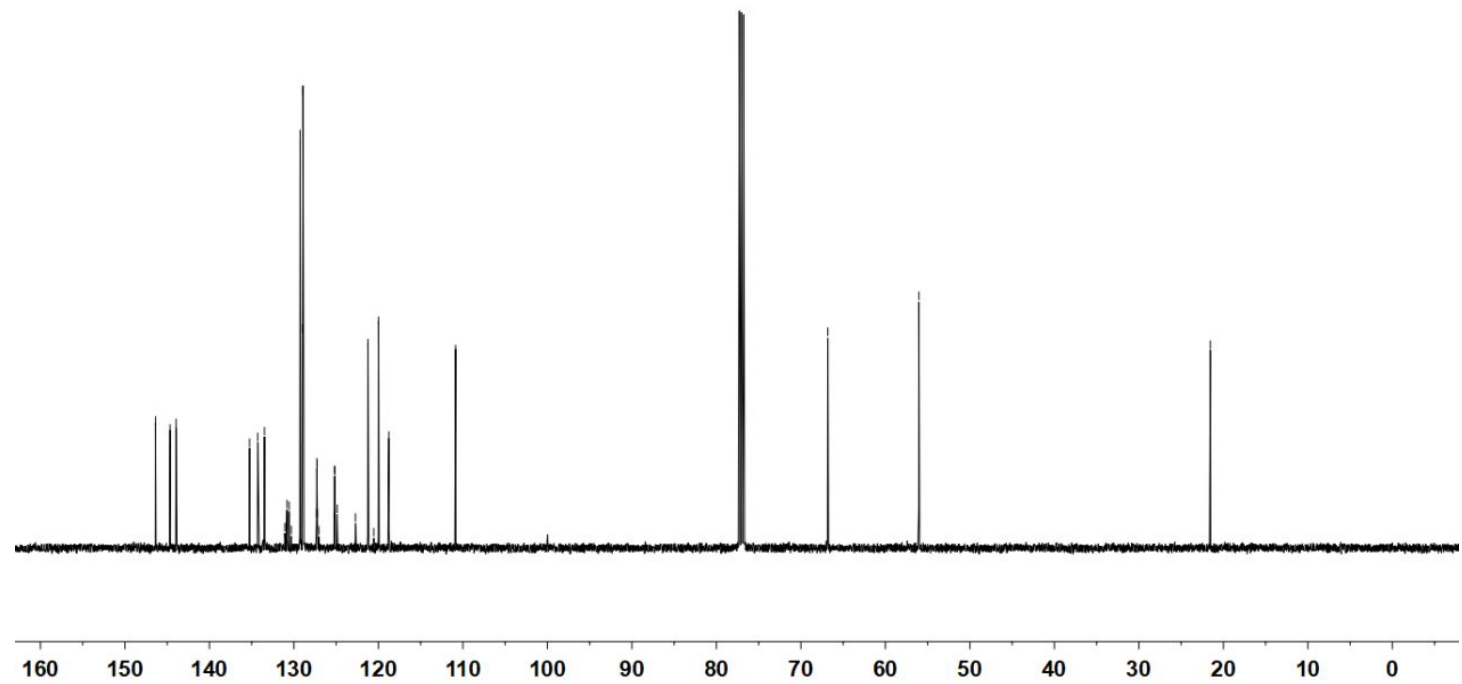<smiles>COc1cccc(C([13CH3])c2ccc(C(F)(F)F)cc2)c1O</smiles>

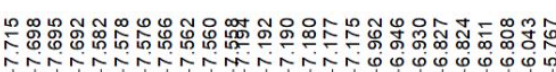

${ }^{1} \mathrm{H}$ NMR (500 MHz, $\left.\mathrm{CDCl}_{3}\right)$

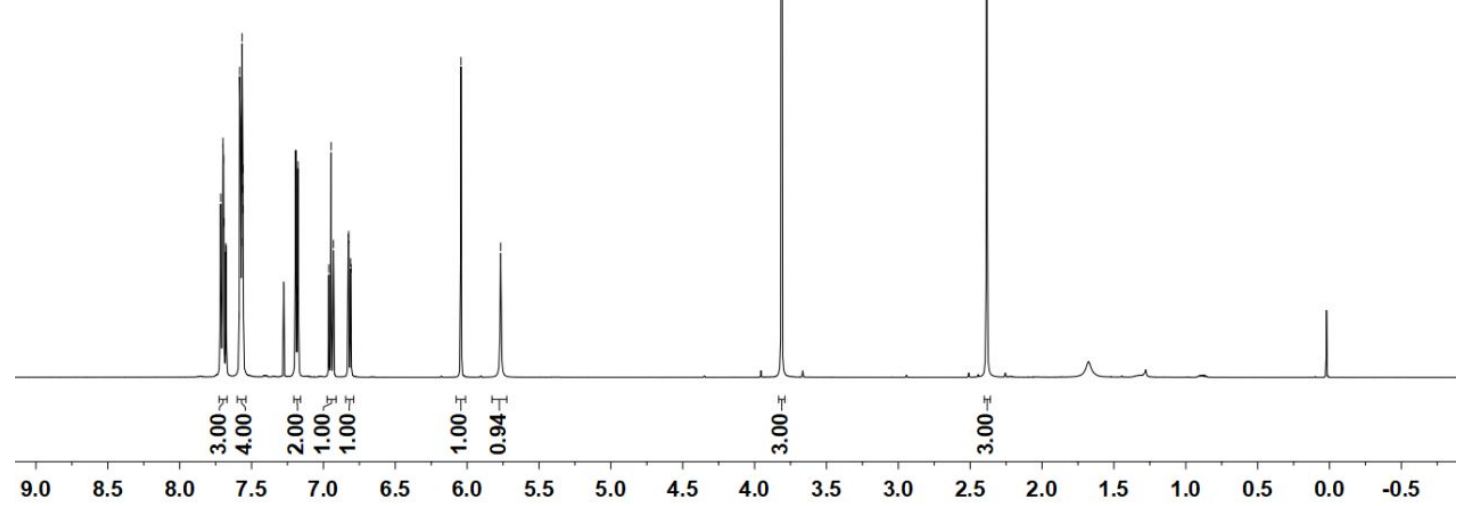



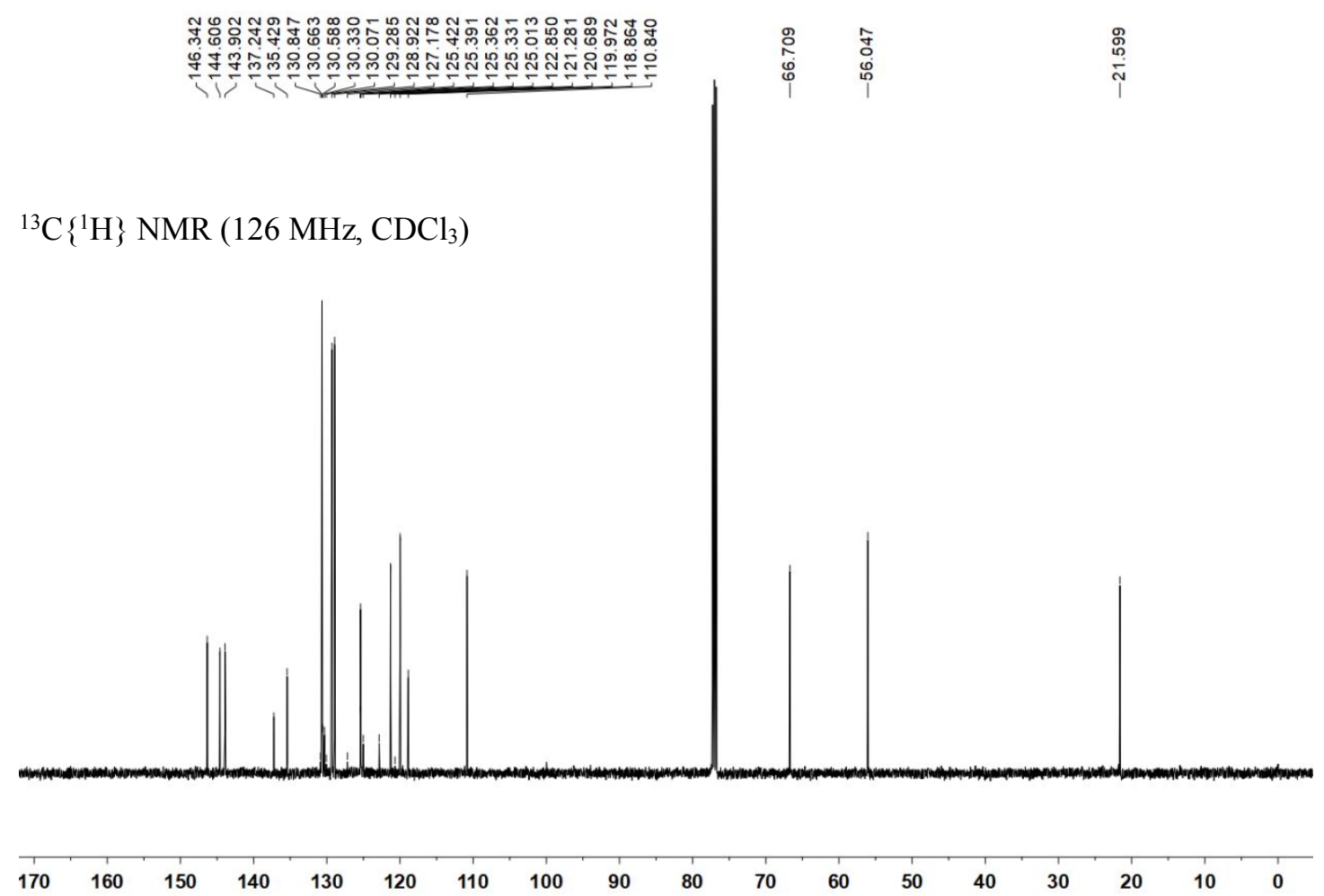<smiles>Oc1cccc(C([18OH])c2ccc(-c3ccccc3)cc2)c1O</smiles>

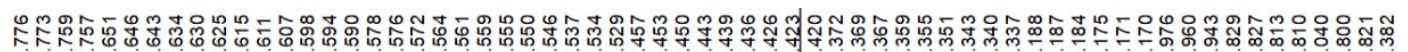

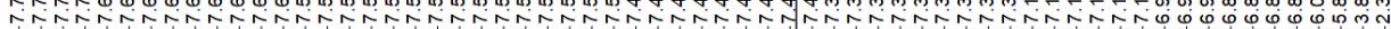

${ }^{1} \mathrm{H}$ NMR $\left(500 \mathrm{MHz}, \mathrm{CDCl}_{3}\right)$

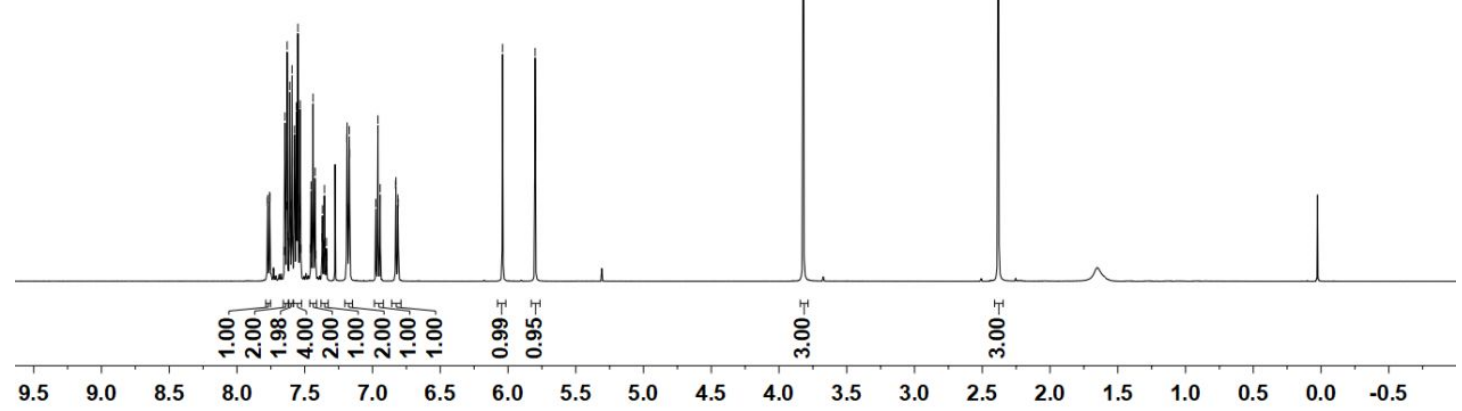




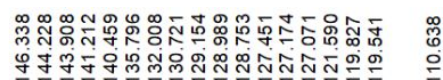

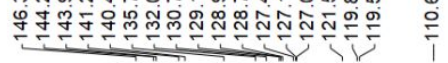

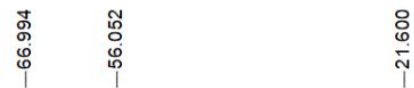

${ }^{13} \mathrm{C}\left\{{ }^{1} \mathrm{H}\right\}$ NMR $\left(126 \mathrm{MHz}, \mathrm{CDCl}_{3}\right)$

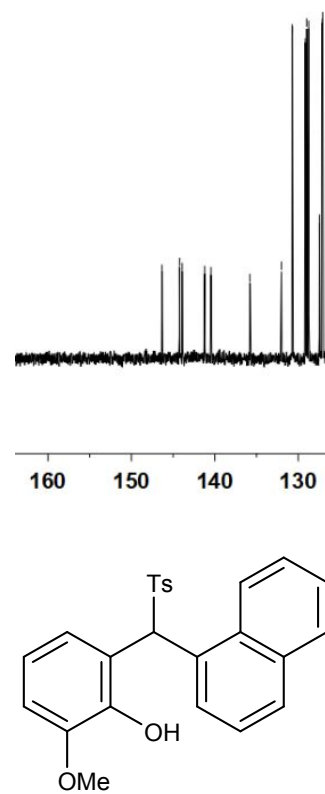

$1 q$

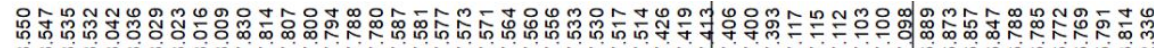

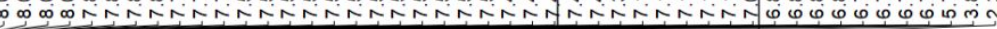

${ }^{1} \mathrm{H} \mathrm{NMR}\left(500 \mathrm{MHz}, \mathrm{CDCl}_{3}\right)$

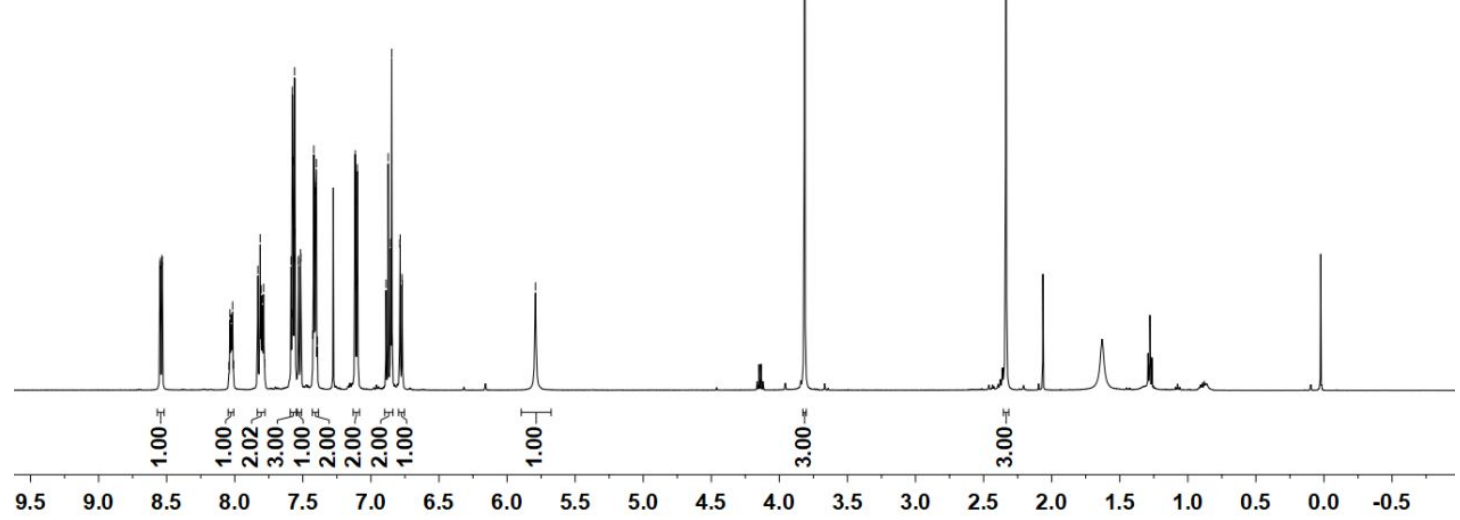



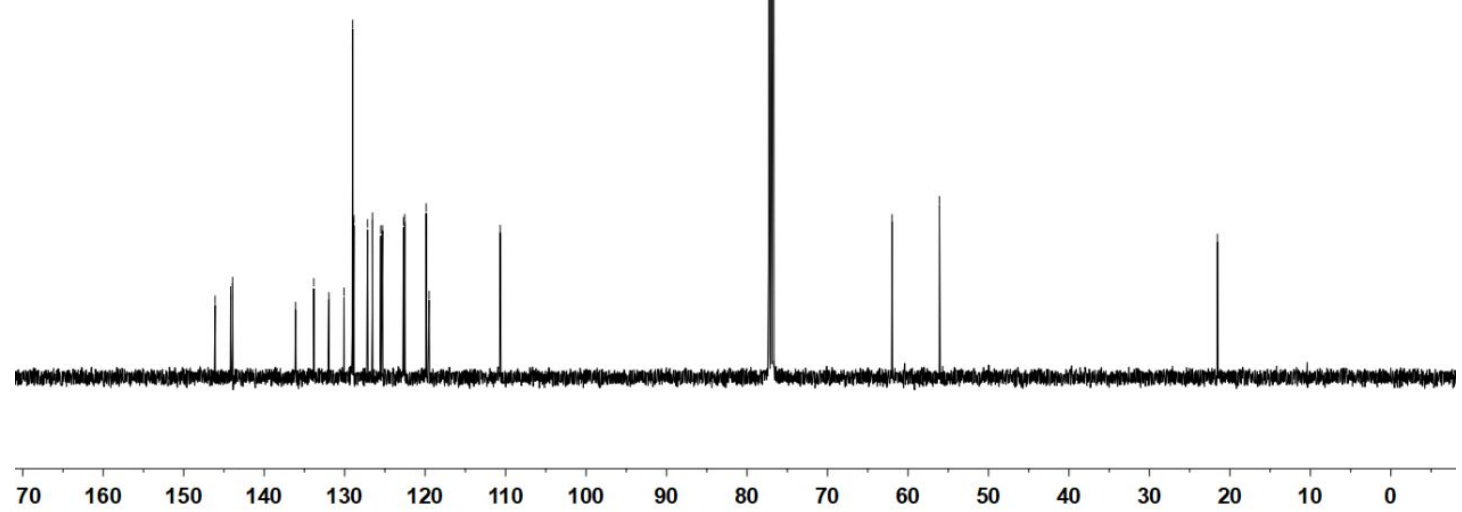<smiles>COc1cccc(C([18OH])c2ccc3ccccc3c2)c1O</smiles>

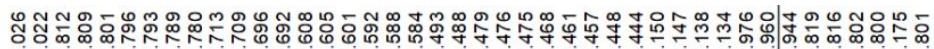

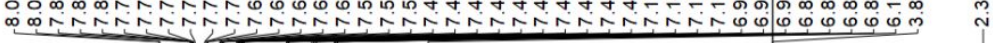

${ }^{1} \mathrm{H}$ NMR (500 MHz, $\left.\mathrm{CDCl}_{3}\right)$

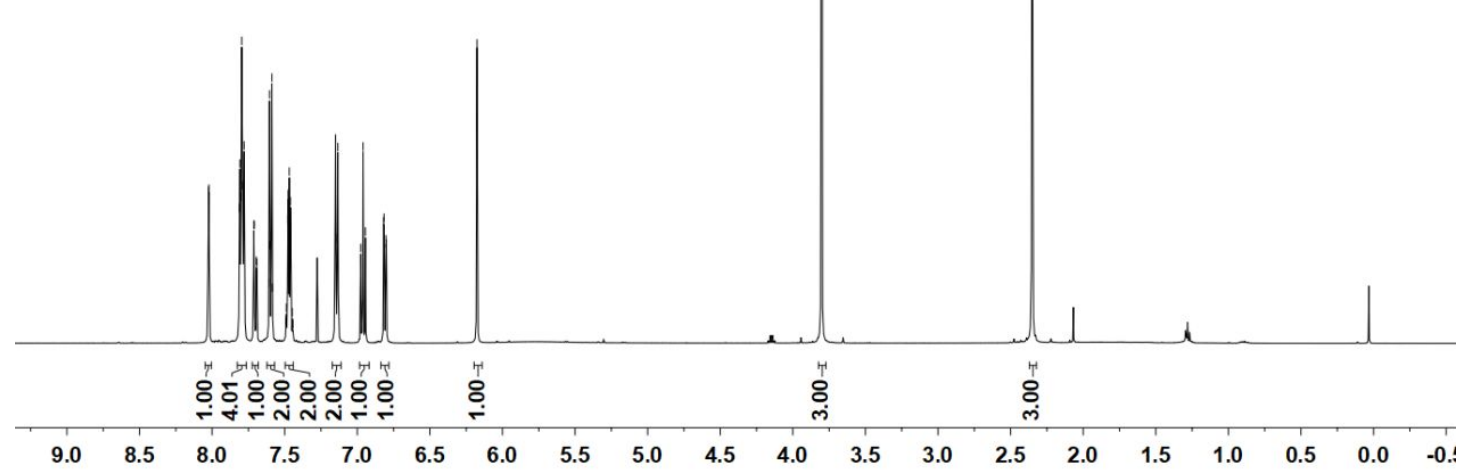



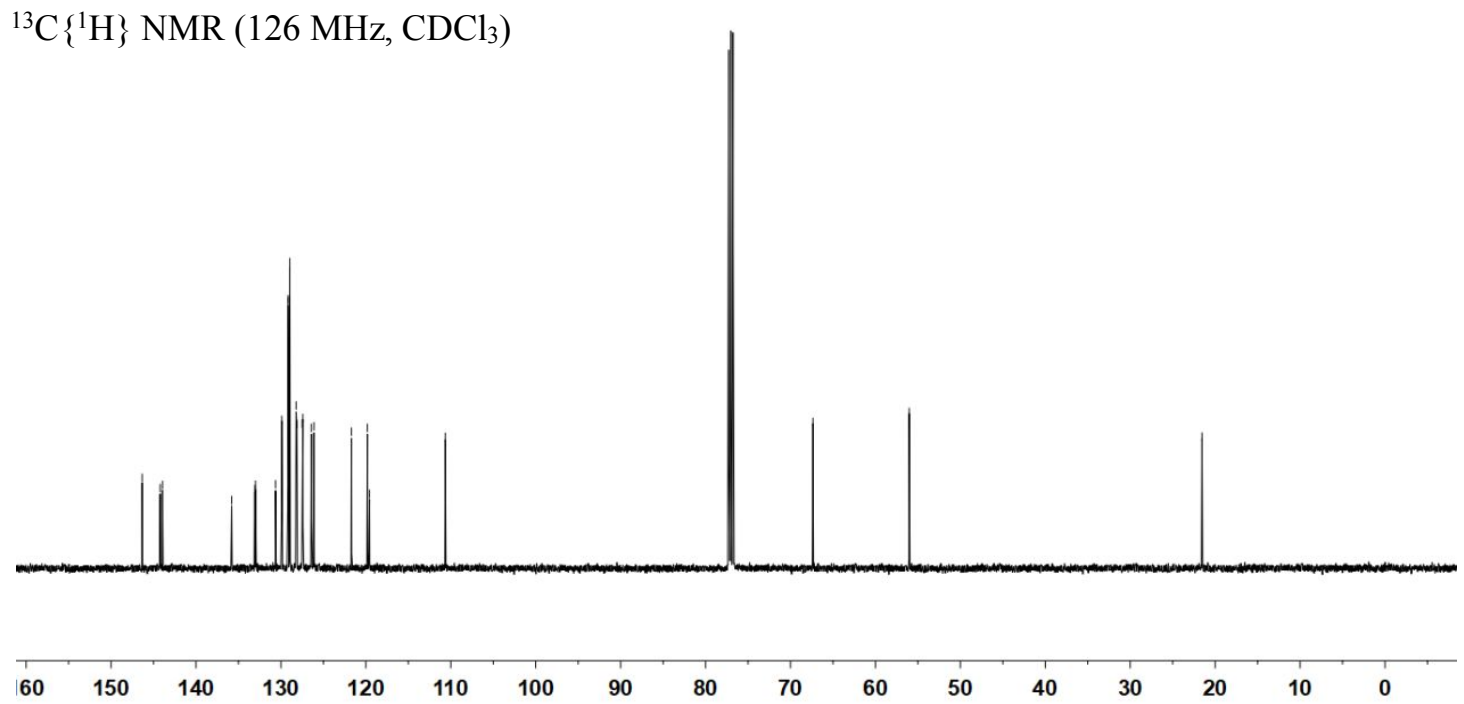<smiles>COc1cccc(C([13CH3])c2ccco2)c1O</smiles>

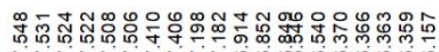

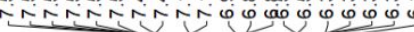

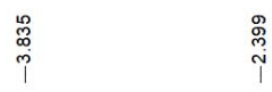

${ }^{1} \mathrm{H}$ NMR (500 MHz, $\mathrm{CDCl}_{3}$ )

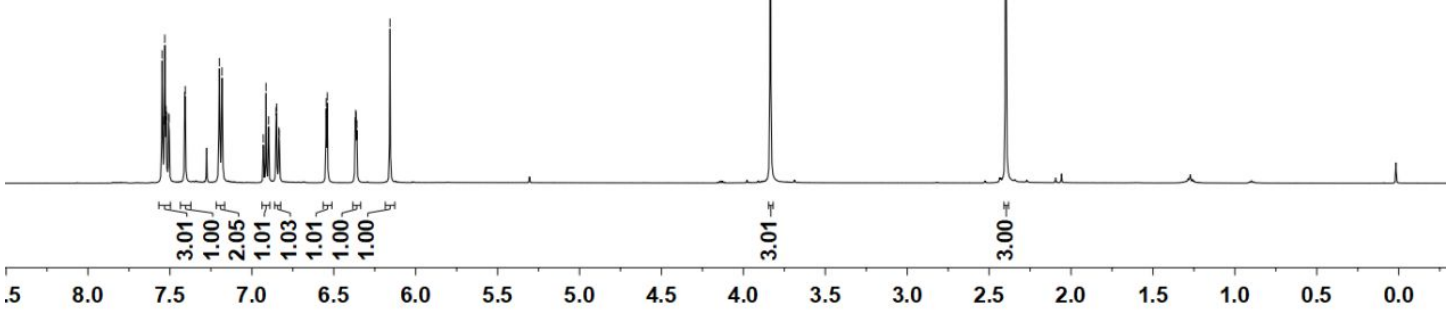


${ }^{13} \mathrm{C}\left\{{ }^{1} \mathrm{H}\right\}$ NMR $\left(126 \mathrm{MHz}, \mathrm{CDCl}_{3}\right)$

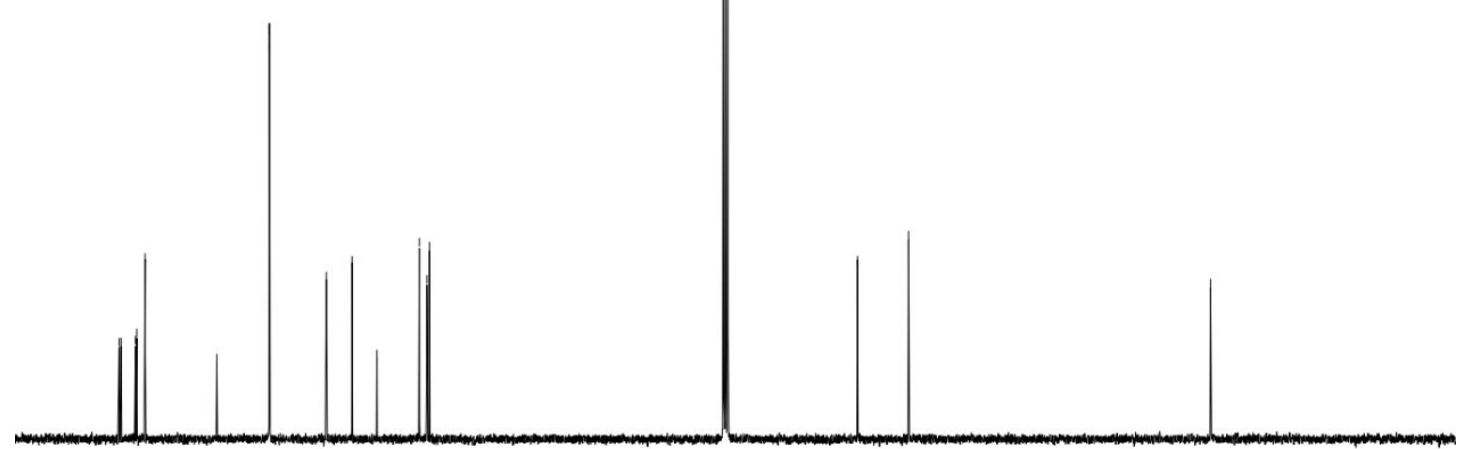

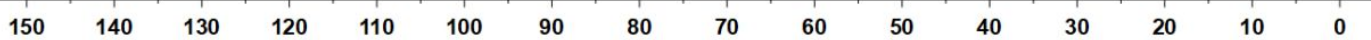<smiles>Oc1cccc(C([18OH])c2cccs2)c1O</smiles>

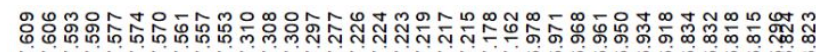

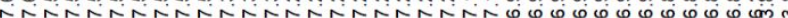

${ }^{1} \mathrm{H}$ NMR $\left(500 \mathrm{MHz}, \mathrm{CDCl}_{3}\right)$

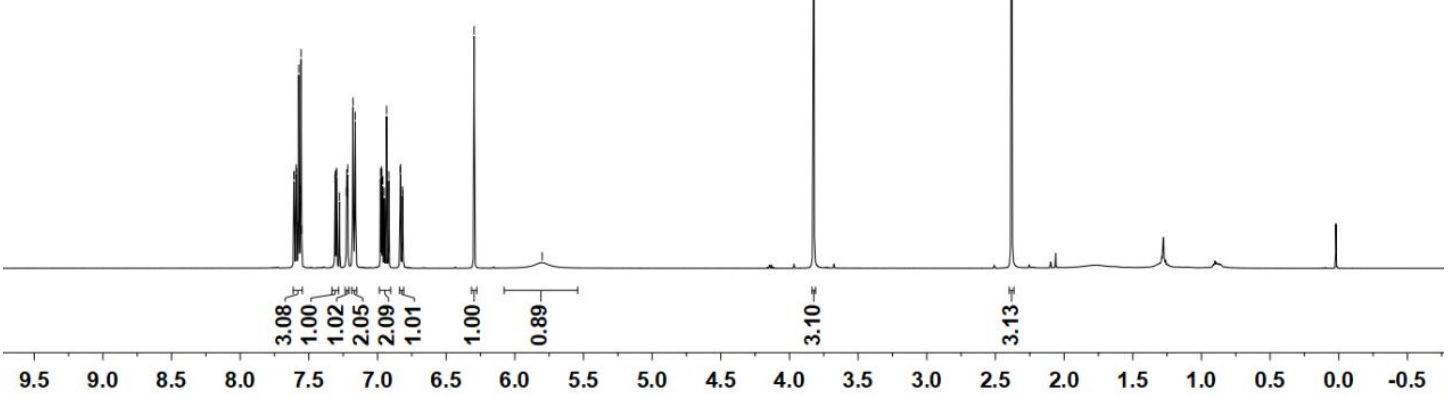




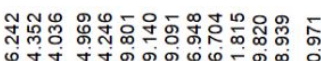

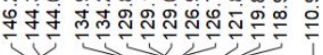

${ }^{13} \mathrm{C}\left\{{ }^{1} \mathrm{H}\right\}$ NMR $\left(126 \mathrm{MHz}, \mathrm{CDCl}_{3}\right)$

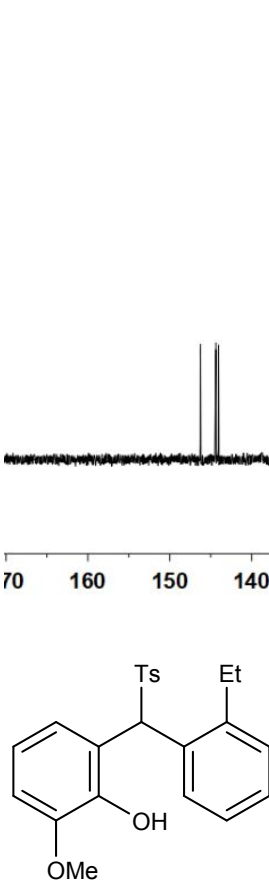

$1 \mathrm{u}$

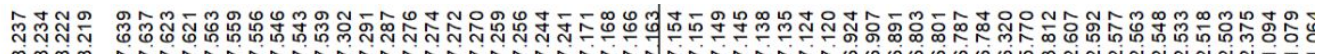

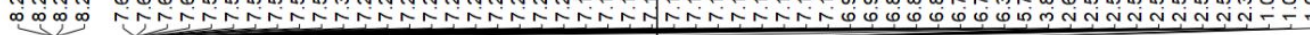

${ }^{1} \mathrm{H}$ NMR (500 MHz, $\left.\mathrm{CDCl}_{3}\right)$

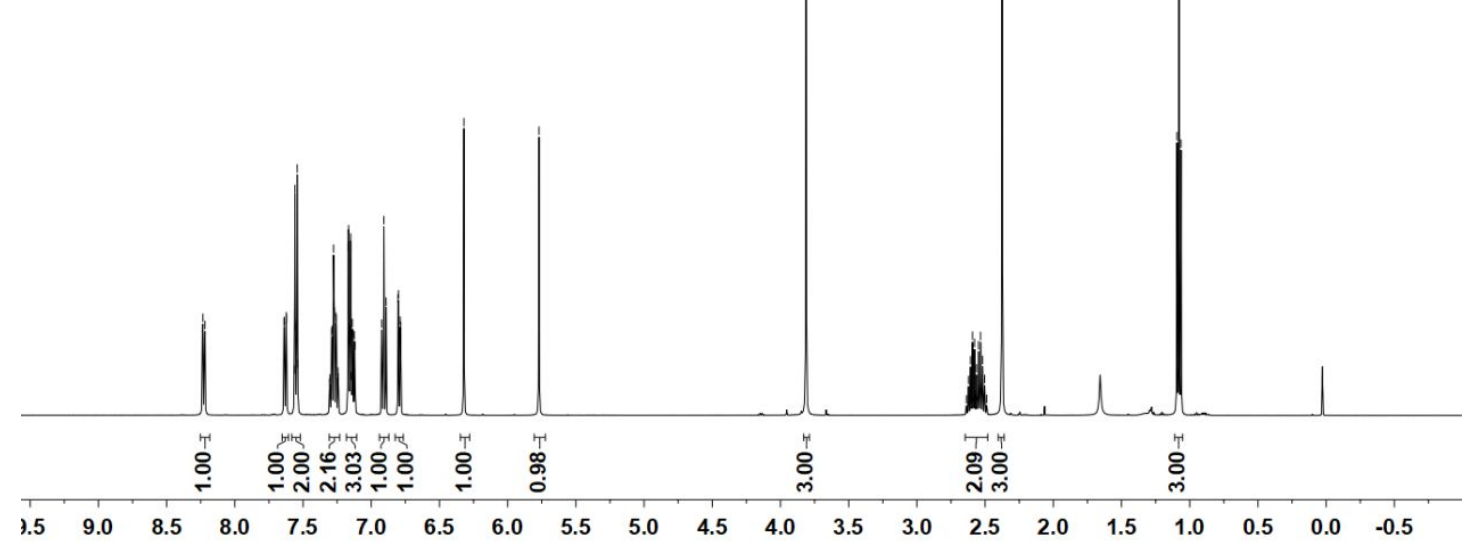




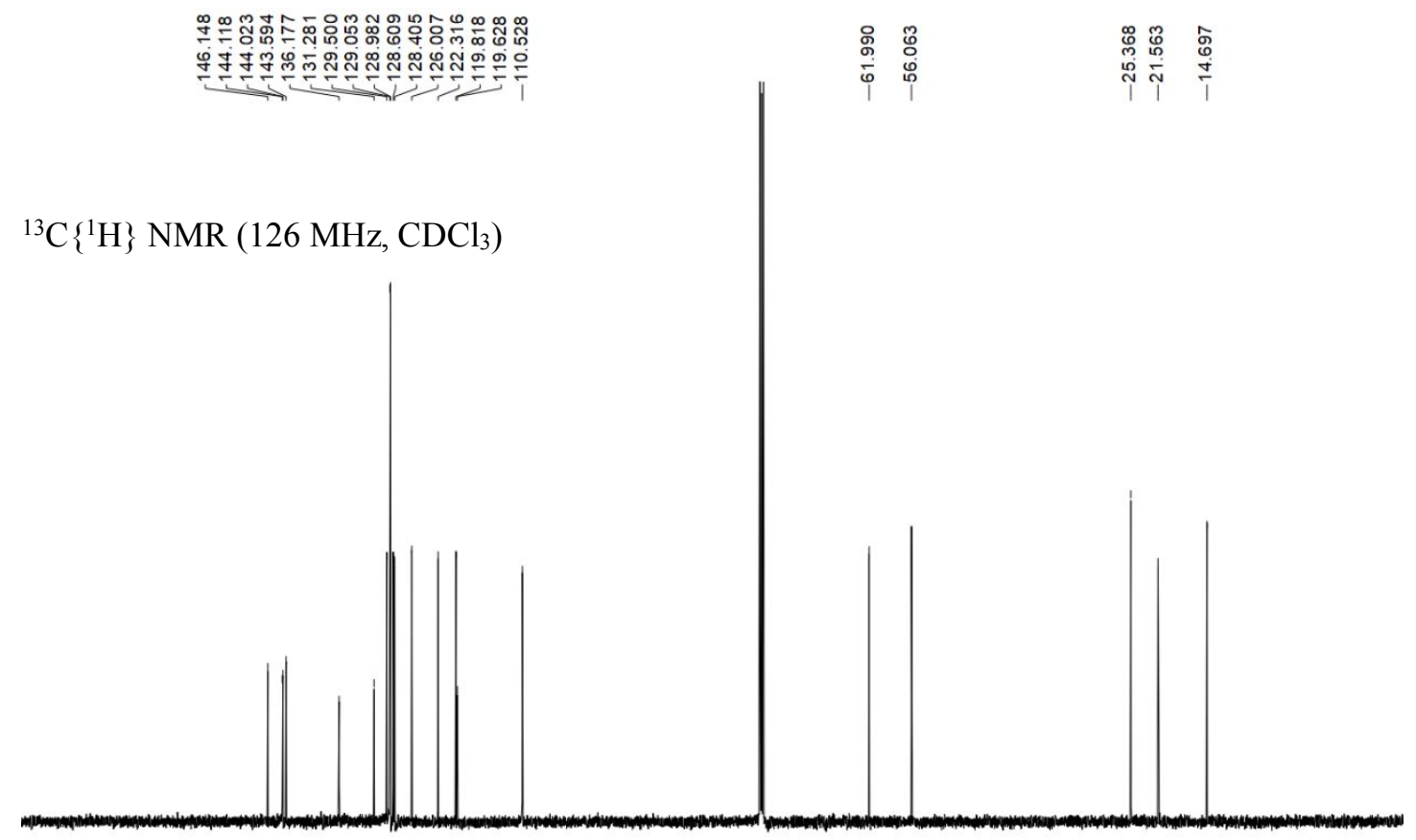

$\begin{array}{llllllllllllllllllll}80 & 170 & 160 & 150 & 140 & 130 & 120 & 110 & 100 & 90 & 80 & 70 & 60 & 50 & 40 & 30 & 20 & 10 & 0 & -10\end{array}$<smiles>COc1cccc([C@H](c2ccccc2)c2ccc3[nH]ccc3c2O)c1</smiles>

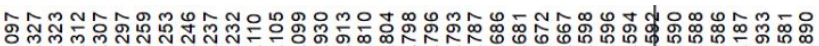

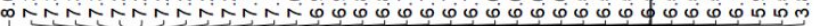

${ }^{1} \mathrm{H}$ NMR (500 MHz, $\left.\mathrm{CDCl}_{3}\right)$

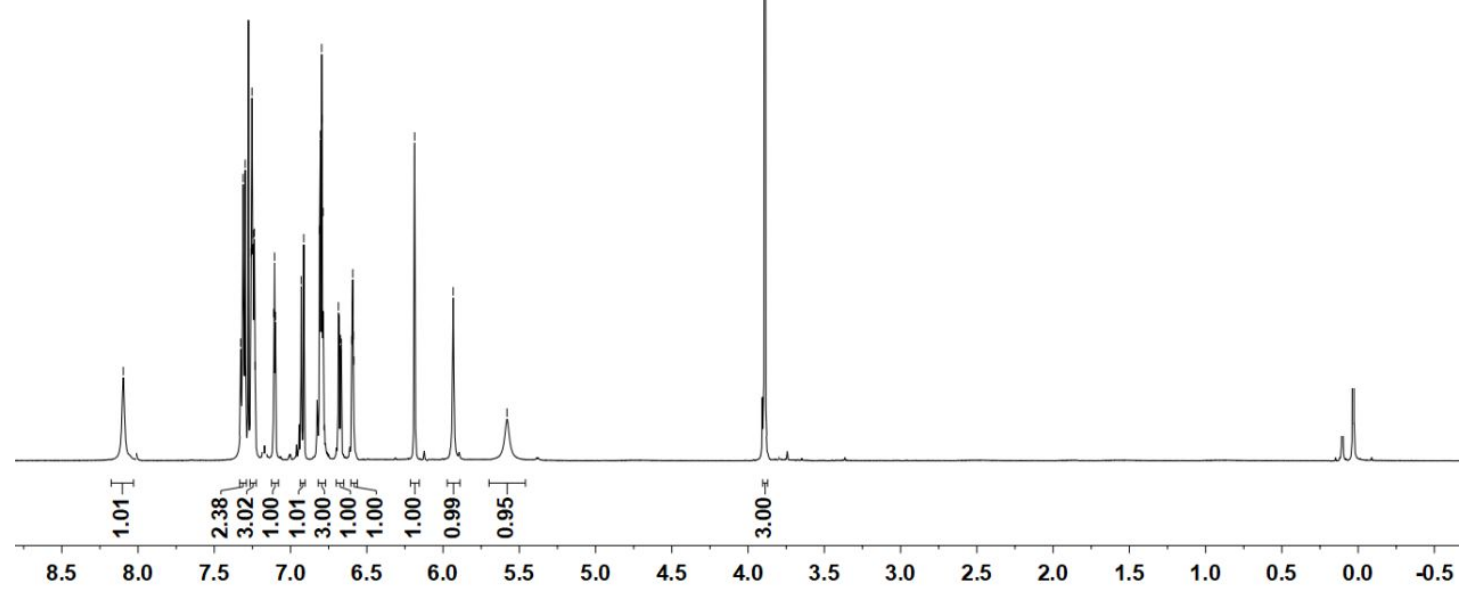




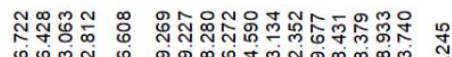

舟守守守

$\stackrel{0}{0}$
$\substack{0 \\ 0}$
0

${ }^{13} \mathrm{C}\left\{{ }^{1} \mathrm{H}\right\}$ NMR $\left(126 \mathrm{MHz}, \mathrm{CDCl}_{3}\right)$
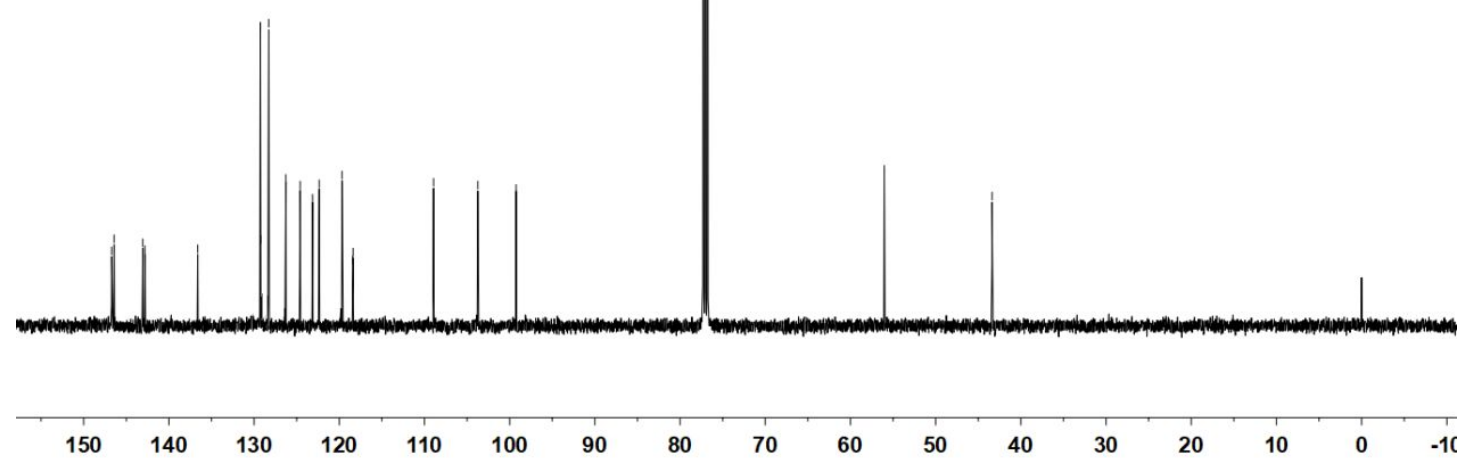<smiles>CCOc1cccc(C(c2ccccc2)c2ccc3[nH]ccc3c2O)c1</smiles>

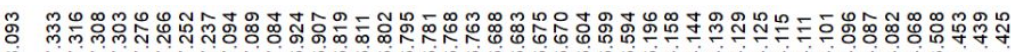

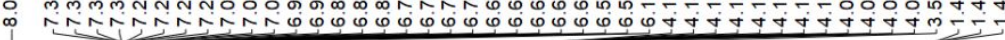

${ }^{1} \mathrm{H}$ NMR $\left(500 \mathrm{MHz}, \mathrm{CDCl}_{3}\right)$

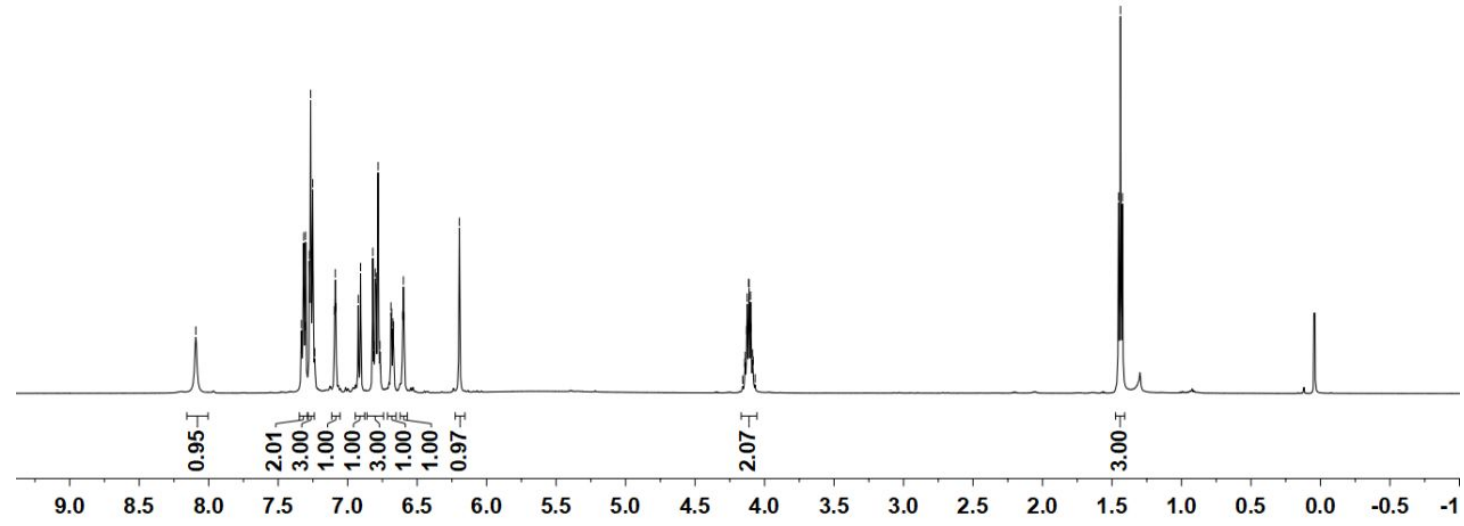




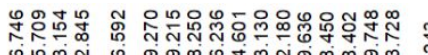

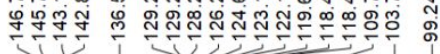

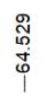

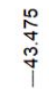

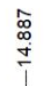

${ }^{13} \mathrm{C}\left\{{ }^{1} \mathrm{H}\right\}$ NMR $\left(126 \mathrm{MHz}, \mathrm{CDCl}_{3}\right)$

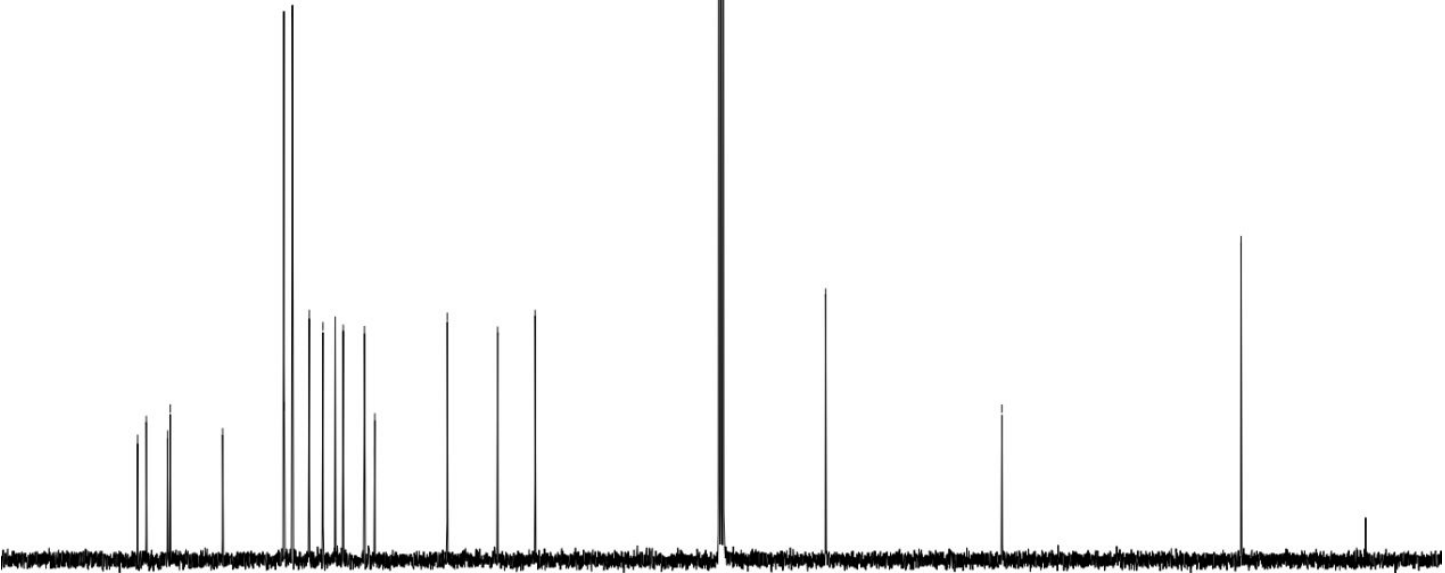

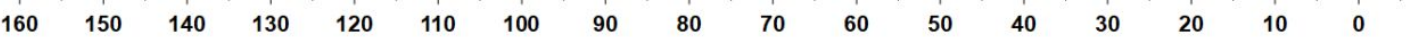<smiles>COc1ccc([C@H](c2ccccc2)c2ccc3[nH]ccc3c2O)c(O)c1</smiles>

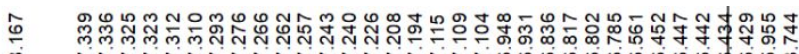

o.

${ }^{1} \mathrm{H}$ NMR $\left(500 \mathrm{MHz}, \mathrm{CDCl}_{3}\right)$

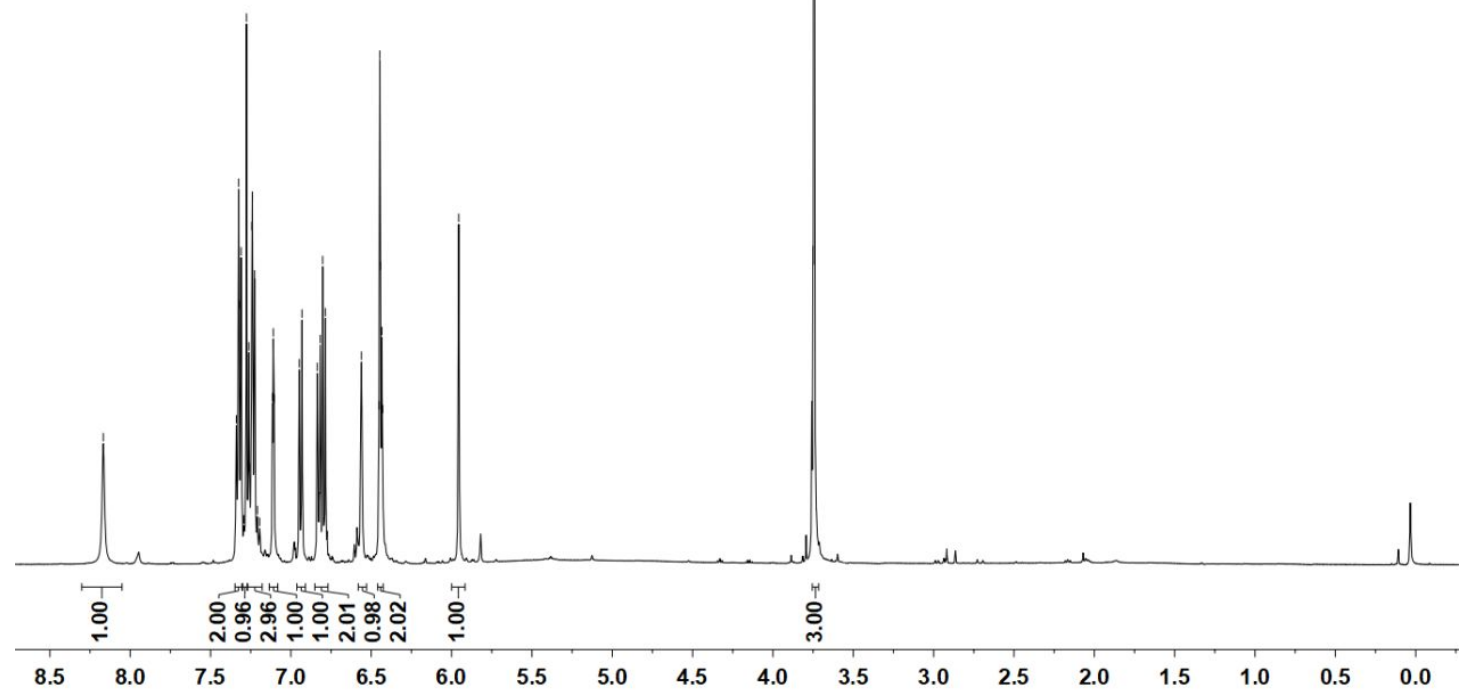


${ }^{13} \mathrm{C}\left\{{ }^{1} \mathrm{H}\right\}$ NMR $\left(126 \mathrm{MHz}, \mathrm{CDCl}_{3}\right)$

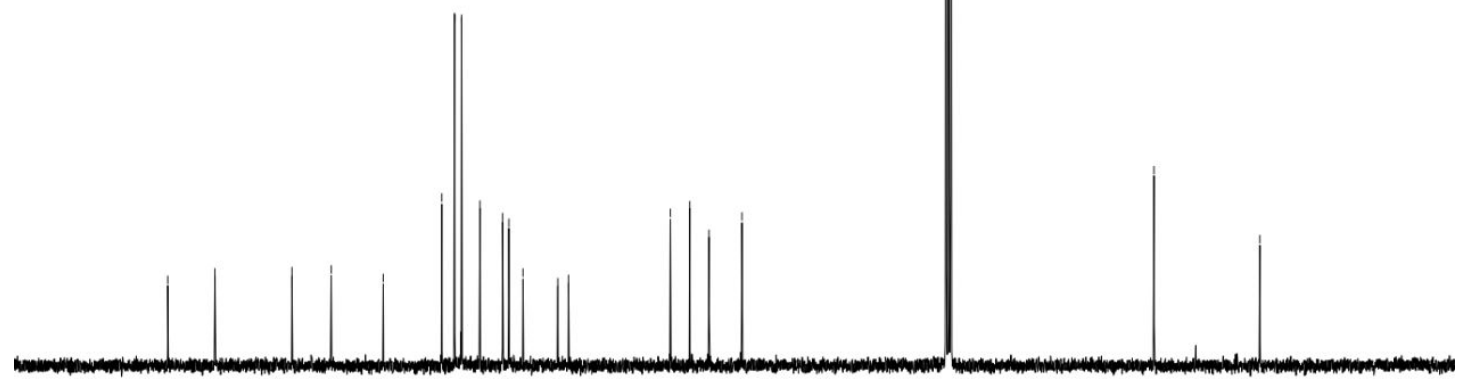<smiles>COc1cc(Br)cc(C(c2ccccc2)c2ccc3[nH]ccc3c2O)c1O</smiles>

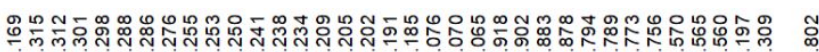

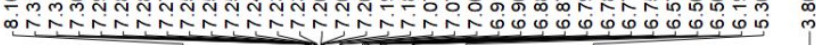

${ }^{1} \mathrm{H} \mathrm{NMR}\left(500 \mathrm{MHz}, \mathrm{CDCl}_{3}\right)$

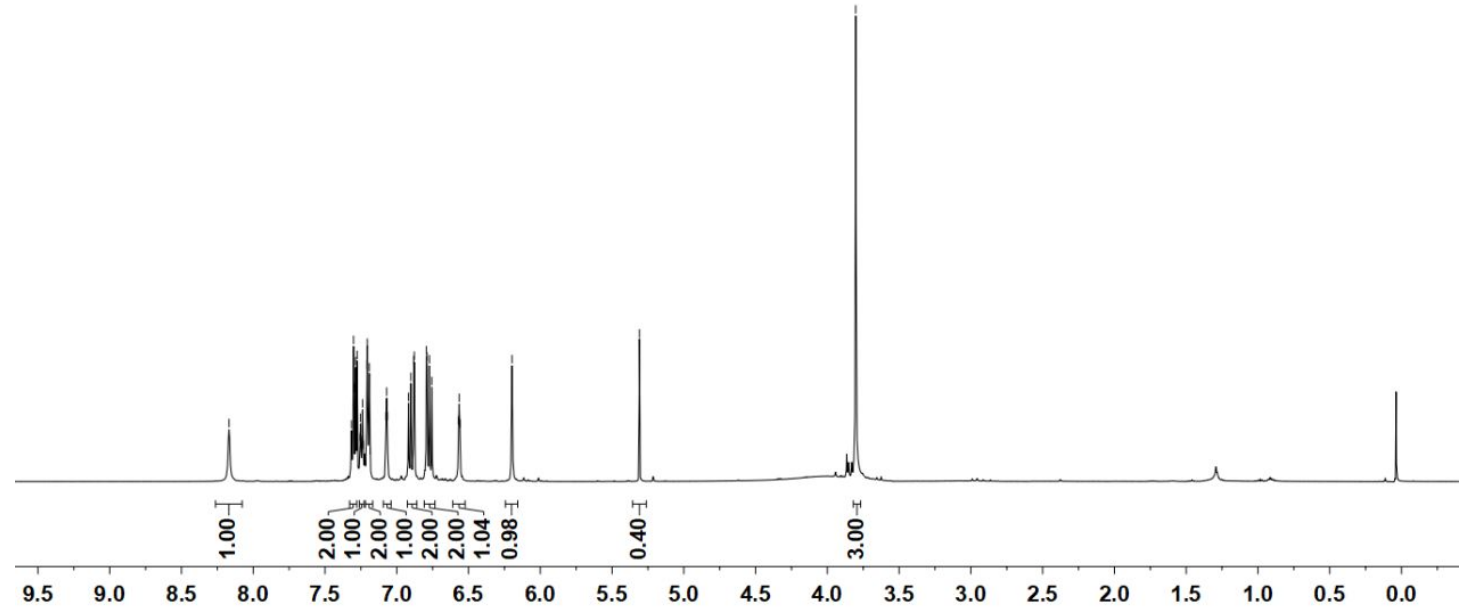




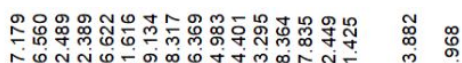

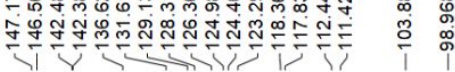

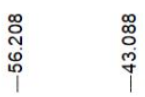

${ }^{13} \mathrm{C}\left\{{ }^{1} \mathrm{H}\right\} \operatorname{NMR}\left(126 \mathrm{MHz}, \mathrm{CDCl}_{3}\right)$
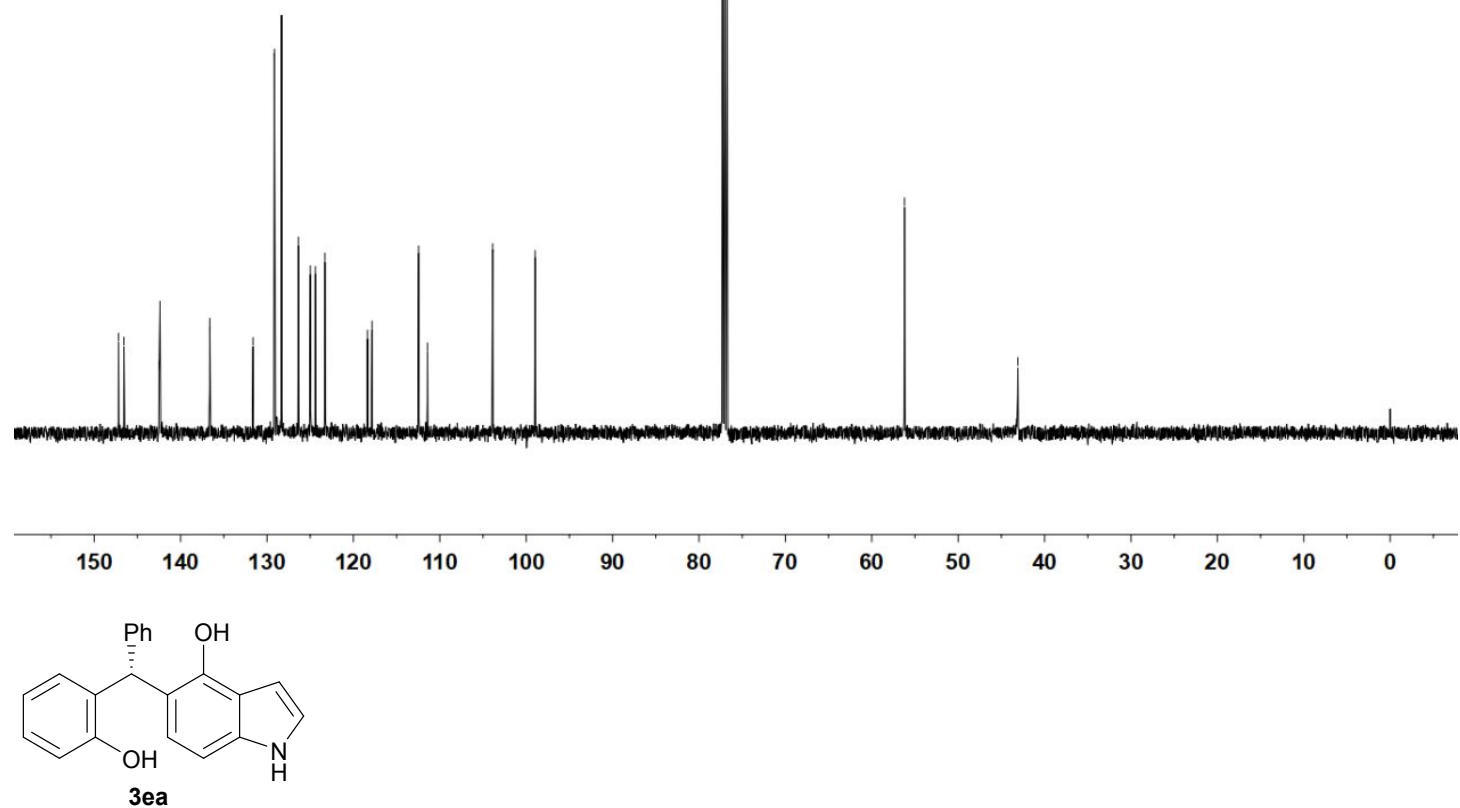

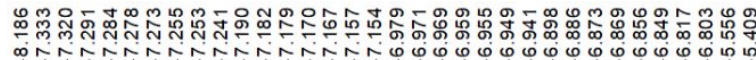

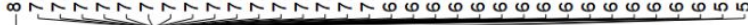

${ }^{1} \mathrm{H}$ NMR $\left(600 \mathrm{MHz}, \mathrm{CDCl}_{3}\right)$

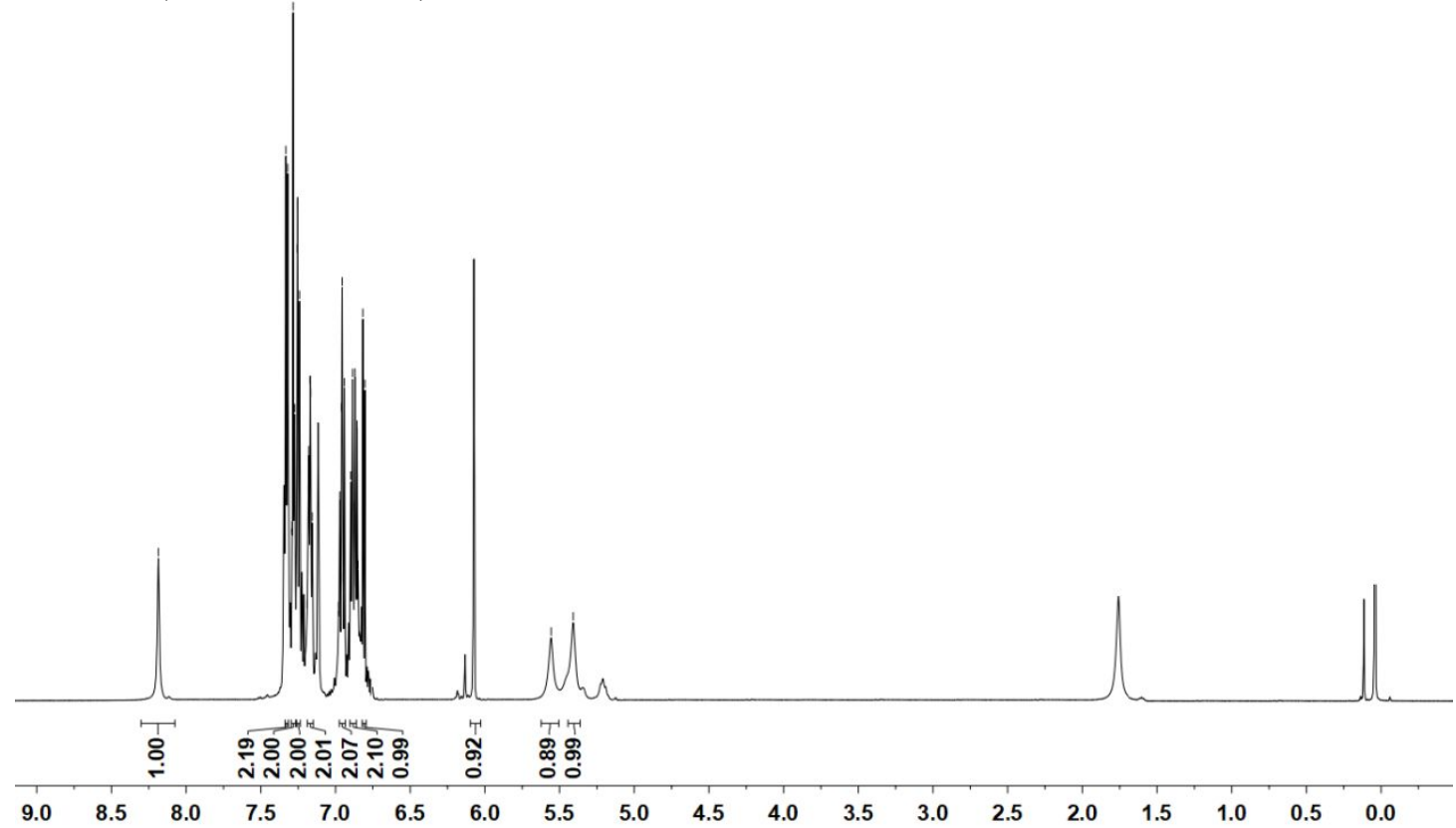



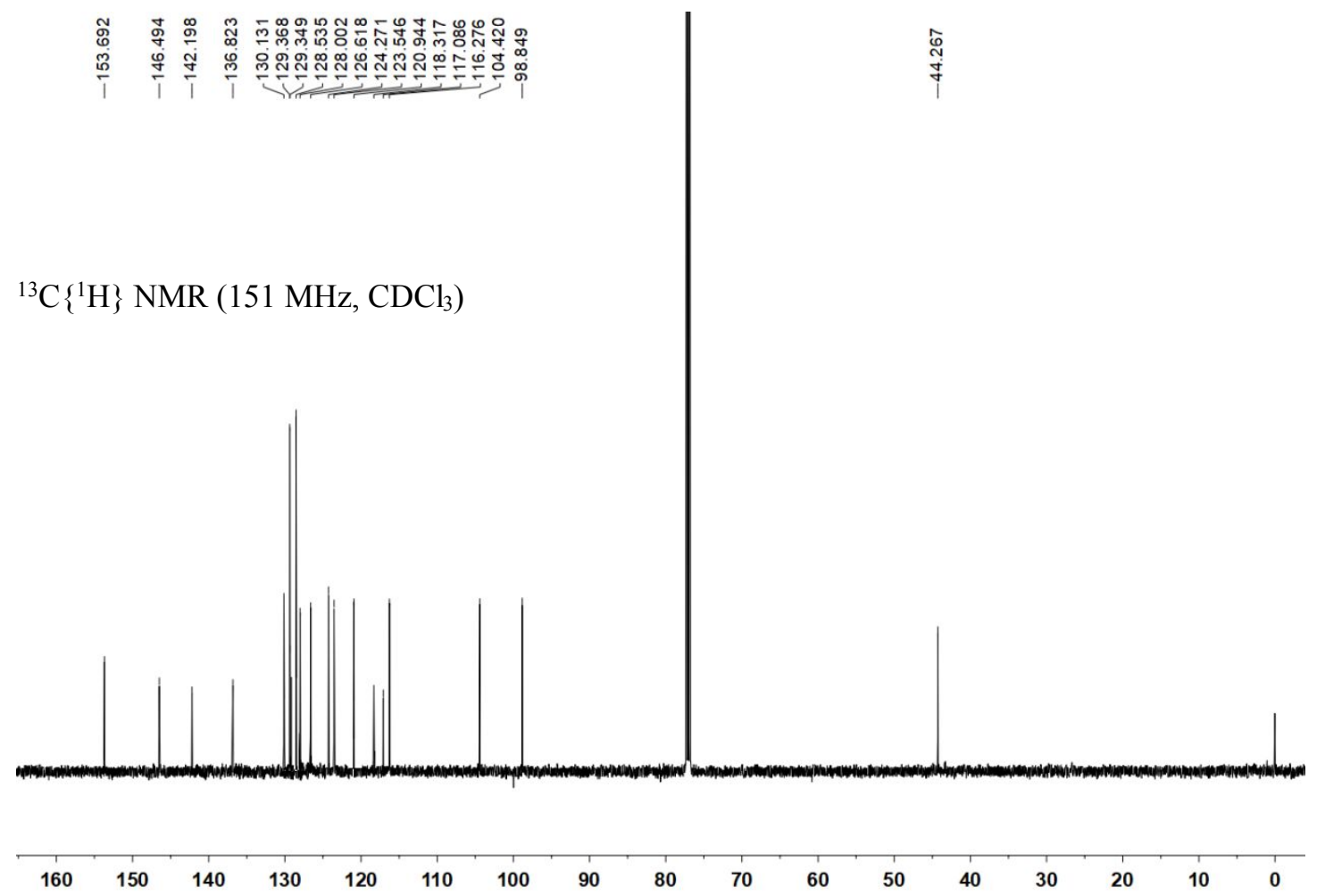<smiles>COc1cccc(C(c2cccc(C)c2)c2ccc3[nH]ccc3c2O)c1</smiles>

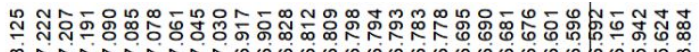

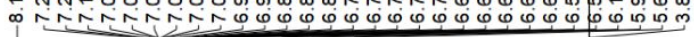

${ }^{1} \mathrm{H}$ NMR $\left(500 \mathrm{MHz}, \mathrm{CDCl}_{3}\right)$

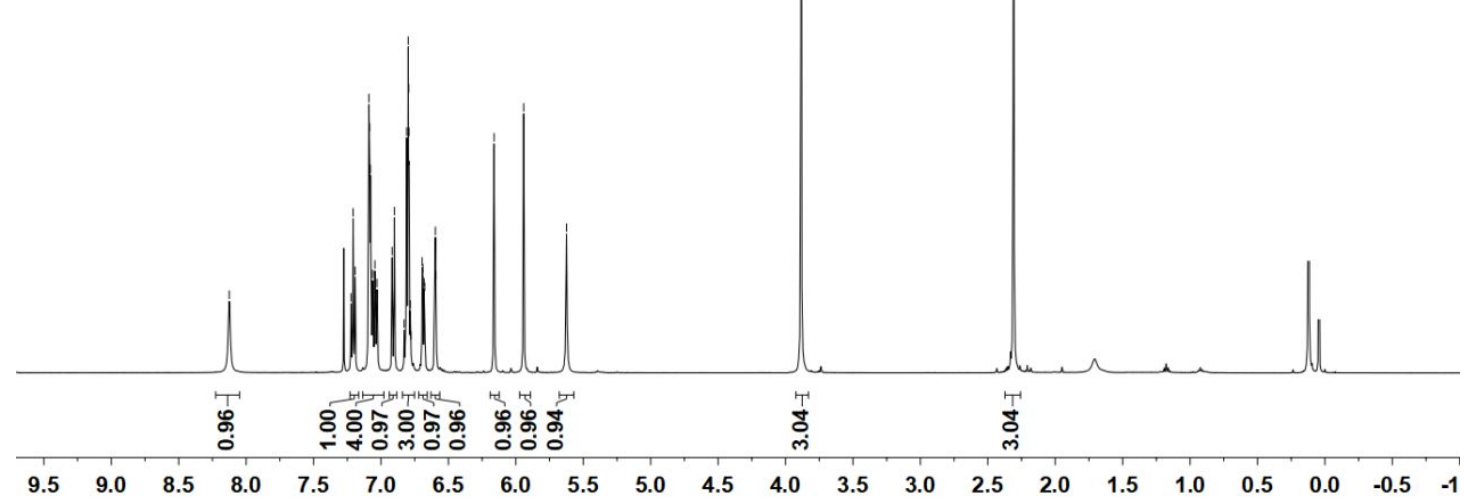


${ }^{13} \mathrm{C}\left\{{ }^{1} \mathrm{H}\right\}$ NMR $\left(126 \mathrm{MHz}, \mathrm{CDCl}_{3}\right)$
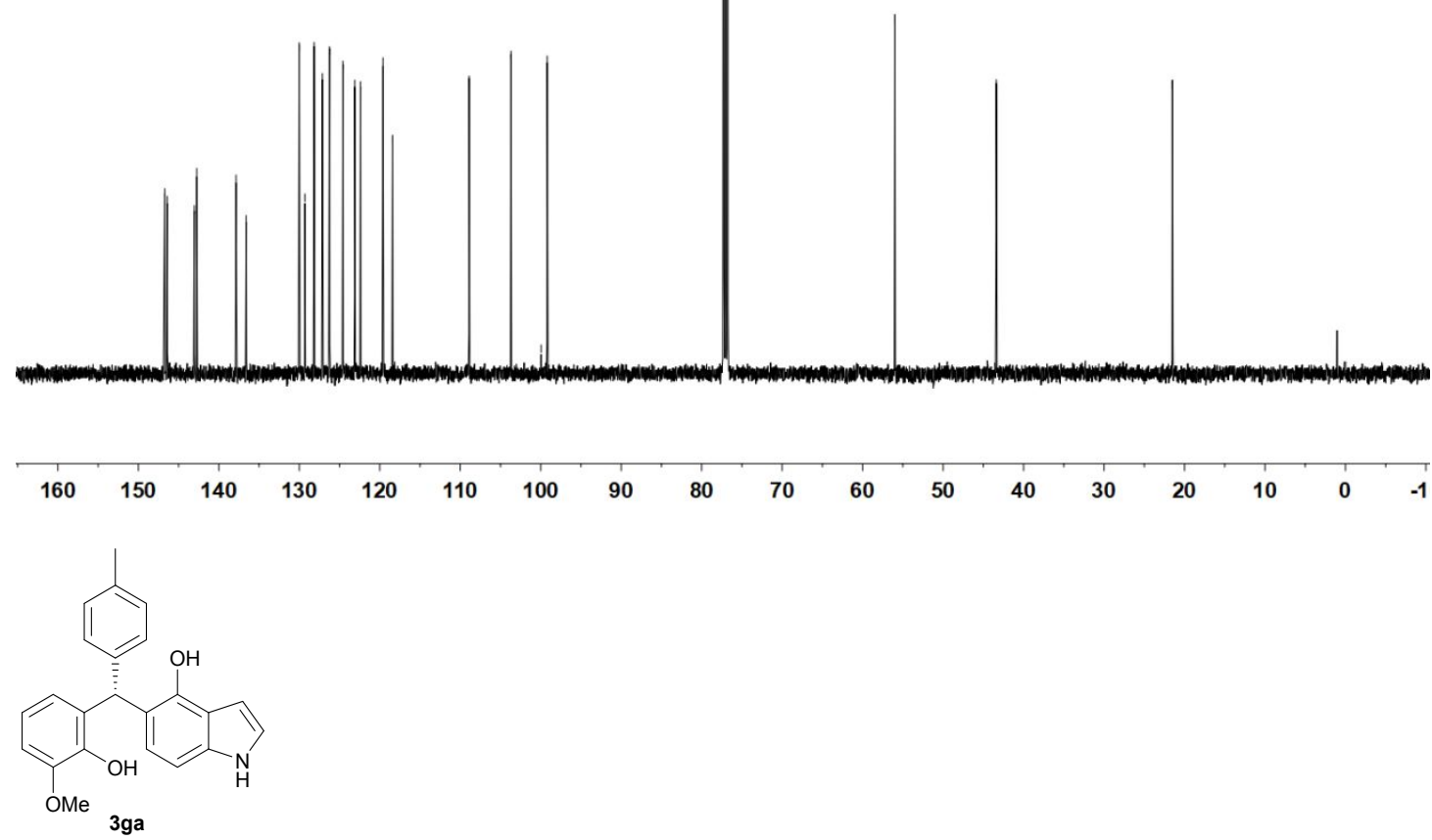

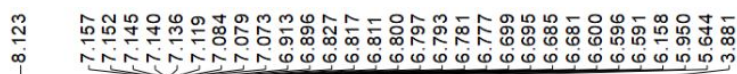

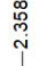

${ }^{1} \mathrm{H}$ NMR (500 MHz, $\mathrm{CDCl}_{3}$ )

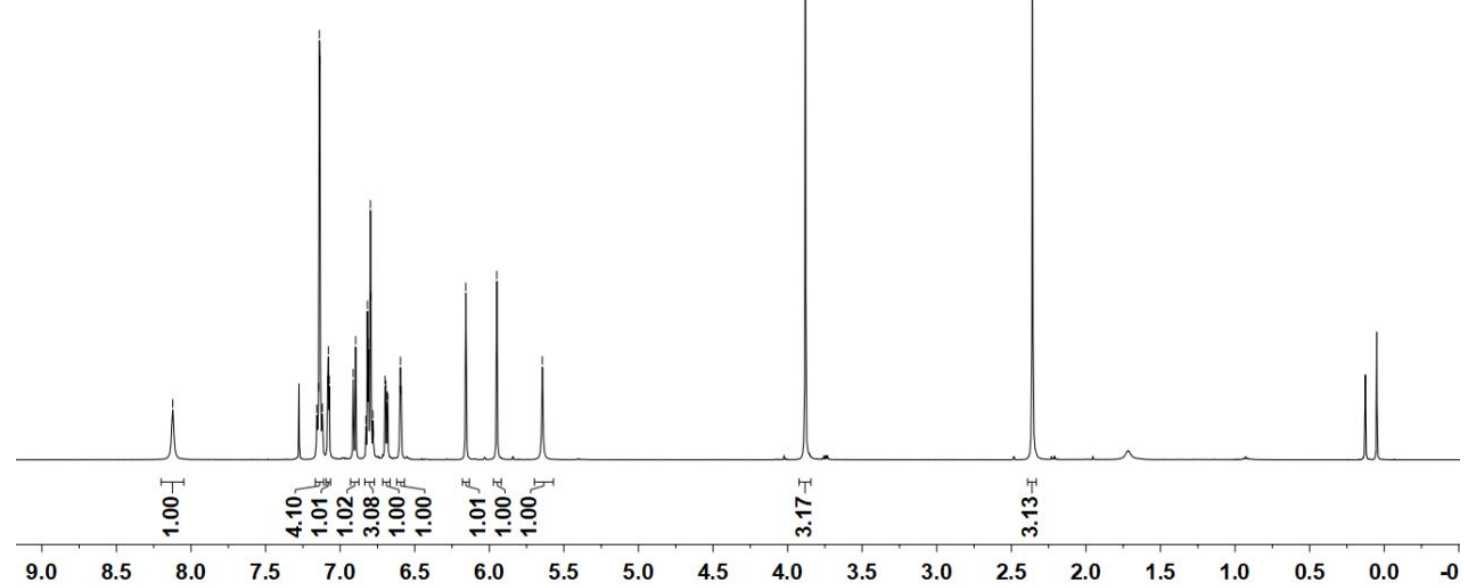



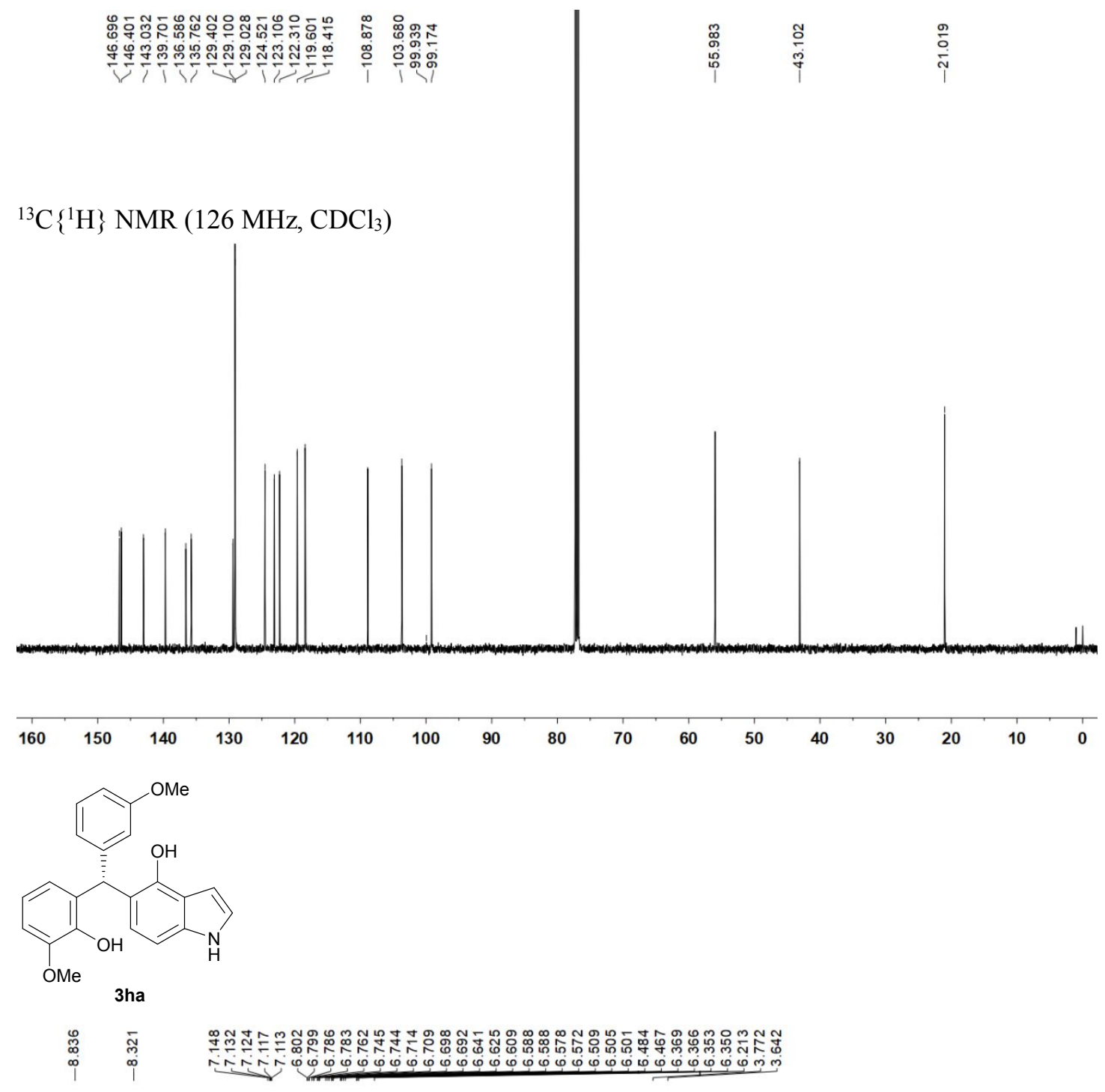

${ }^{1} \mathrm{H}$ NMR (500 MHz, DMSO- $d_{6}$ )

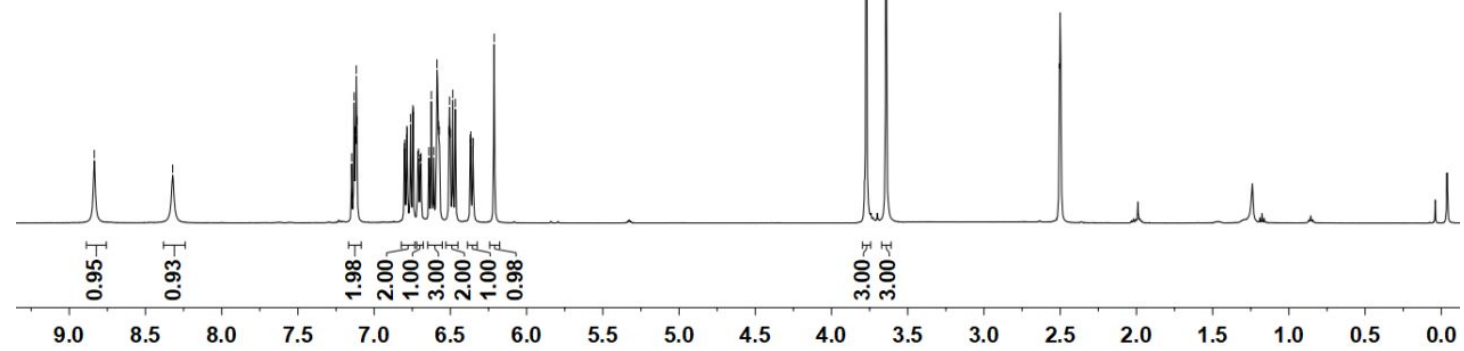


\begin{tabular}{l}
1 \\
\hline
\end{tabular}

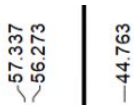

${ }^{13} \mathrm{C}\left\{{ }^{1} \mathrm{H}\right\}$ NMR $\left(126 \mathrm{MHz}, \mathrm{CD}_{3} \mathrm{OD}-d_{4}\right)$
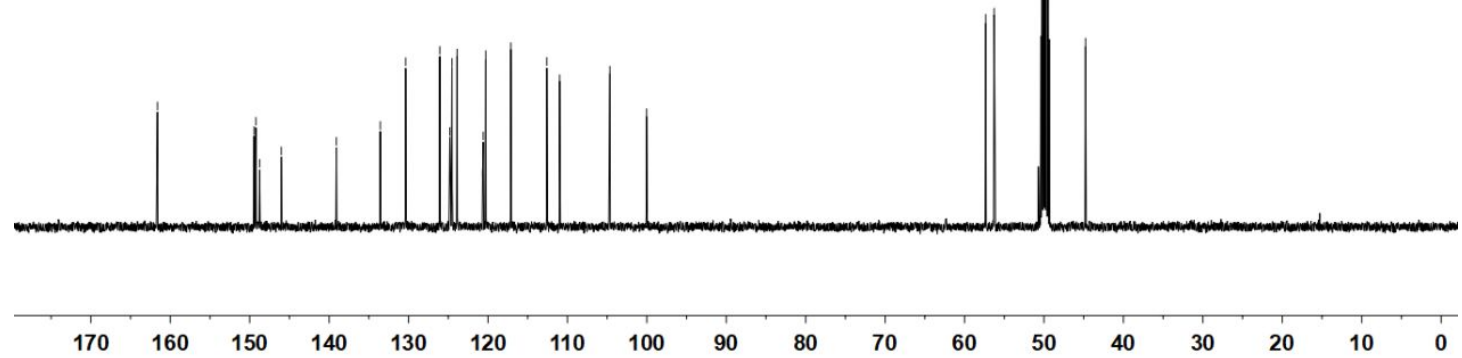<smiles>COc1cccc(C(c2cccc(OC(F)(F)F)c2)c2ccc3[nH]ccc3c2O)c1O</smiles>

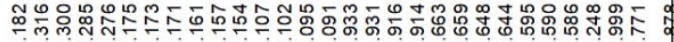

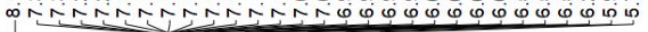

${ }^{1} \mathrm{H}$ NMR $\left(500 \mathrm{MHz}, \mathrm{CDCl}_{3}\right)$

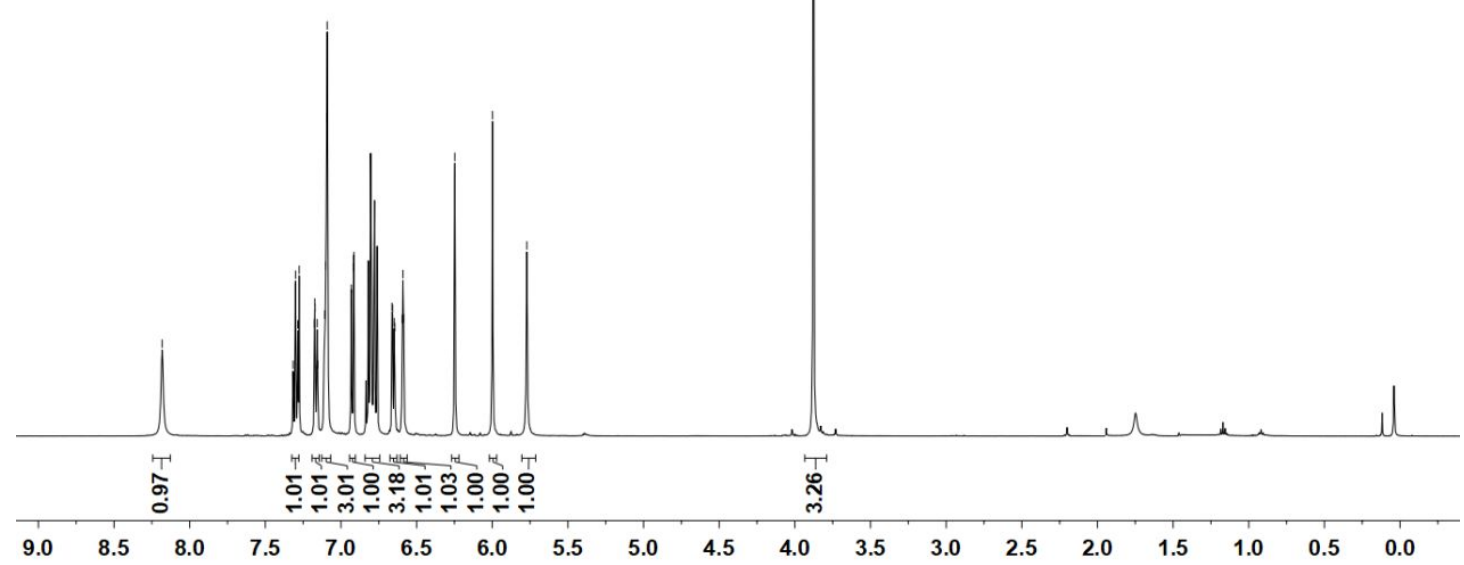


${ }^{13} \mathrm{C}\left\{{ }^{1} \mathrm{H}\right\}$ NMR $\left(126 \mathrm{MHz}, \mathrm{CDCl}_{3}\right)$

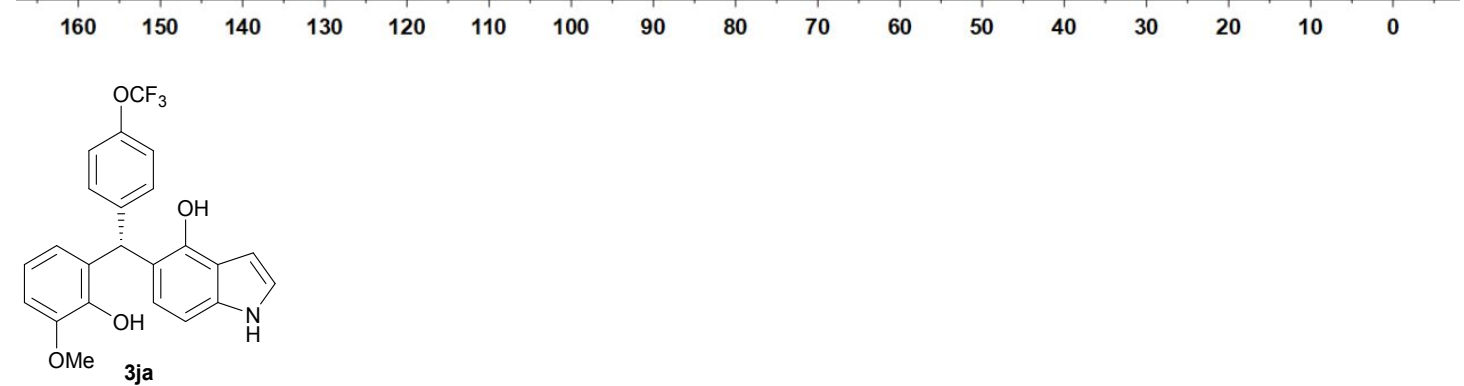

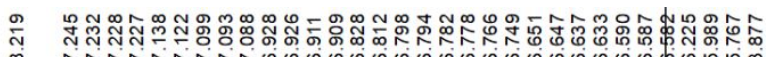

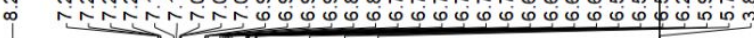

${ }^{1} \mathrm{H}$ NMR $\left(500 \mathrm{MHz}, \mathrm{CDCl}_{3}\right)$

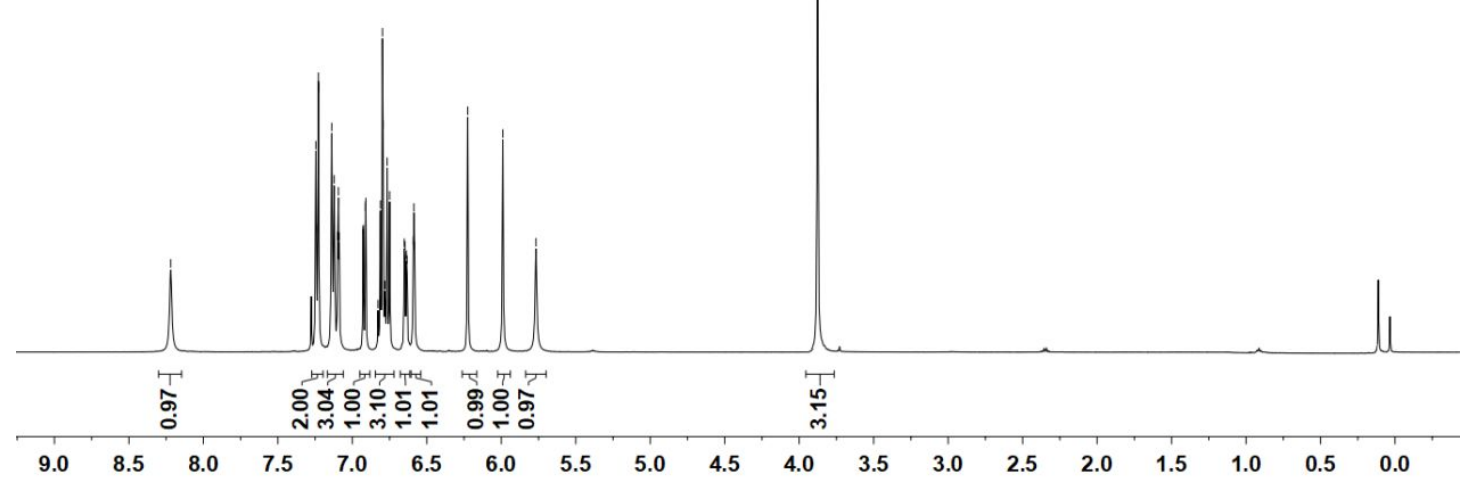


${ }^{13} \mathrm{C}\left\{{ }^{1} \mathrm{H}\right\}$ NMR (126 MHz, $\left.\mathrm{CDCl}_{3}\right)$
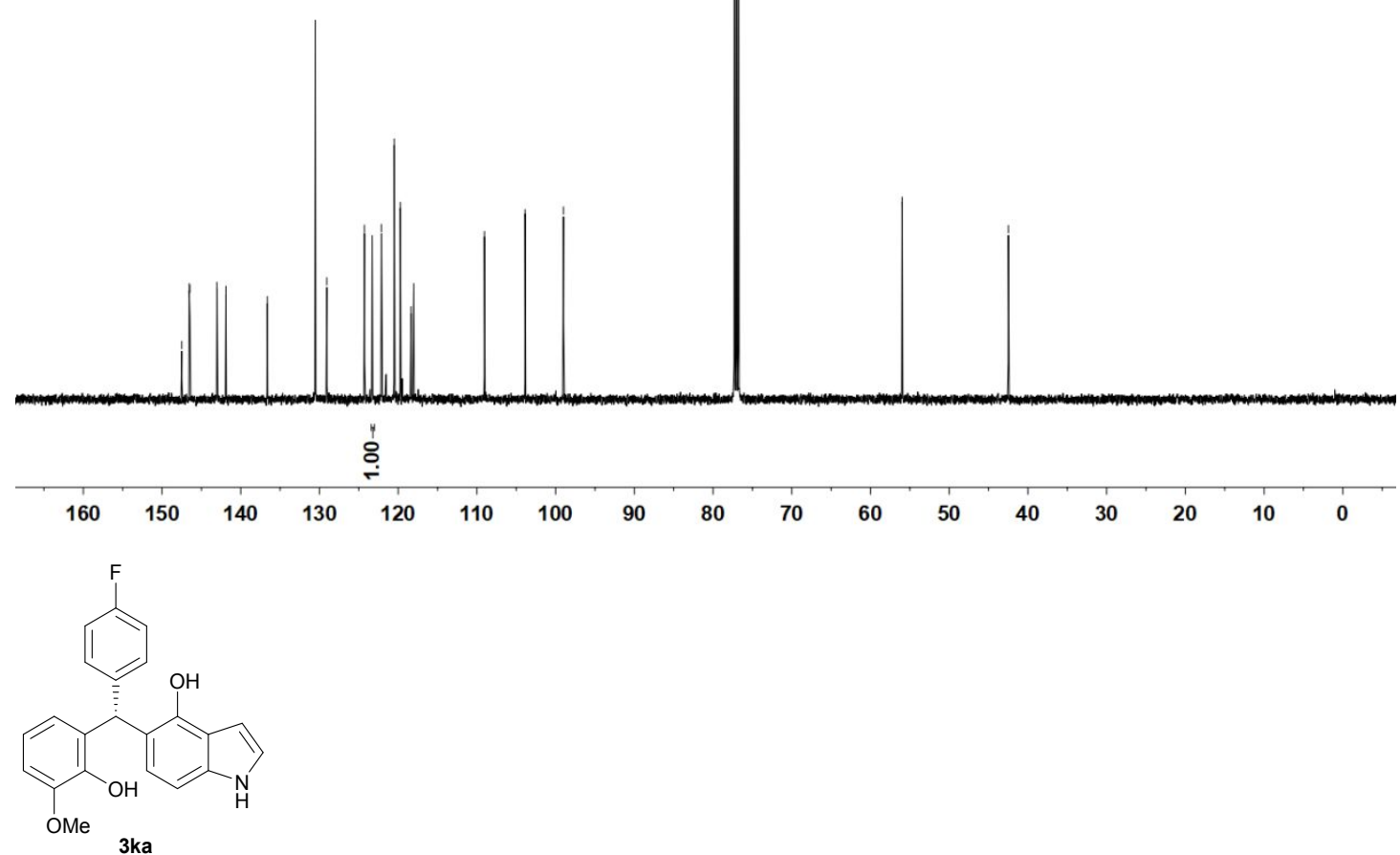

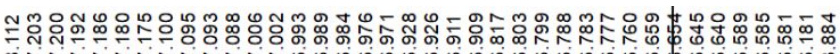

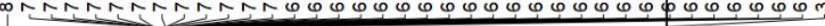

${ }^{1} \mathrm{H}$ NMR $\left(500 \mathrm{MHz}, \mathrm{CDCl}_{3}\right)$

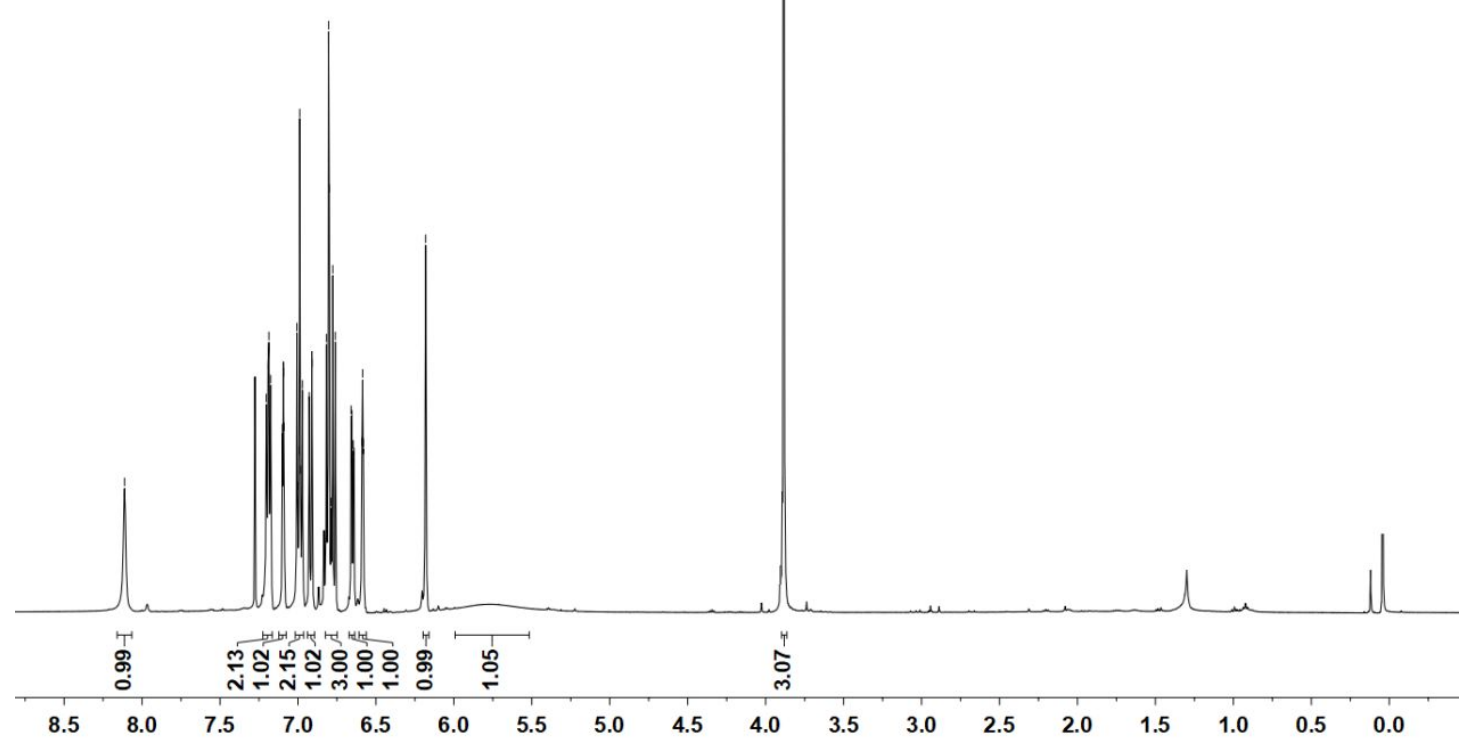



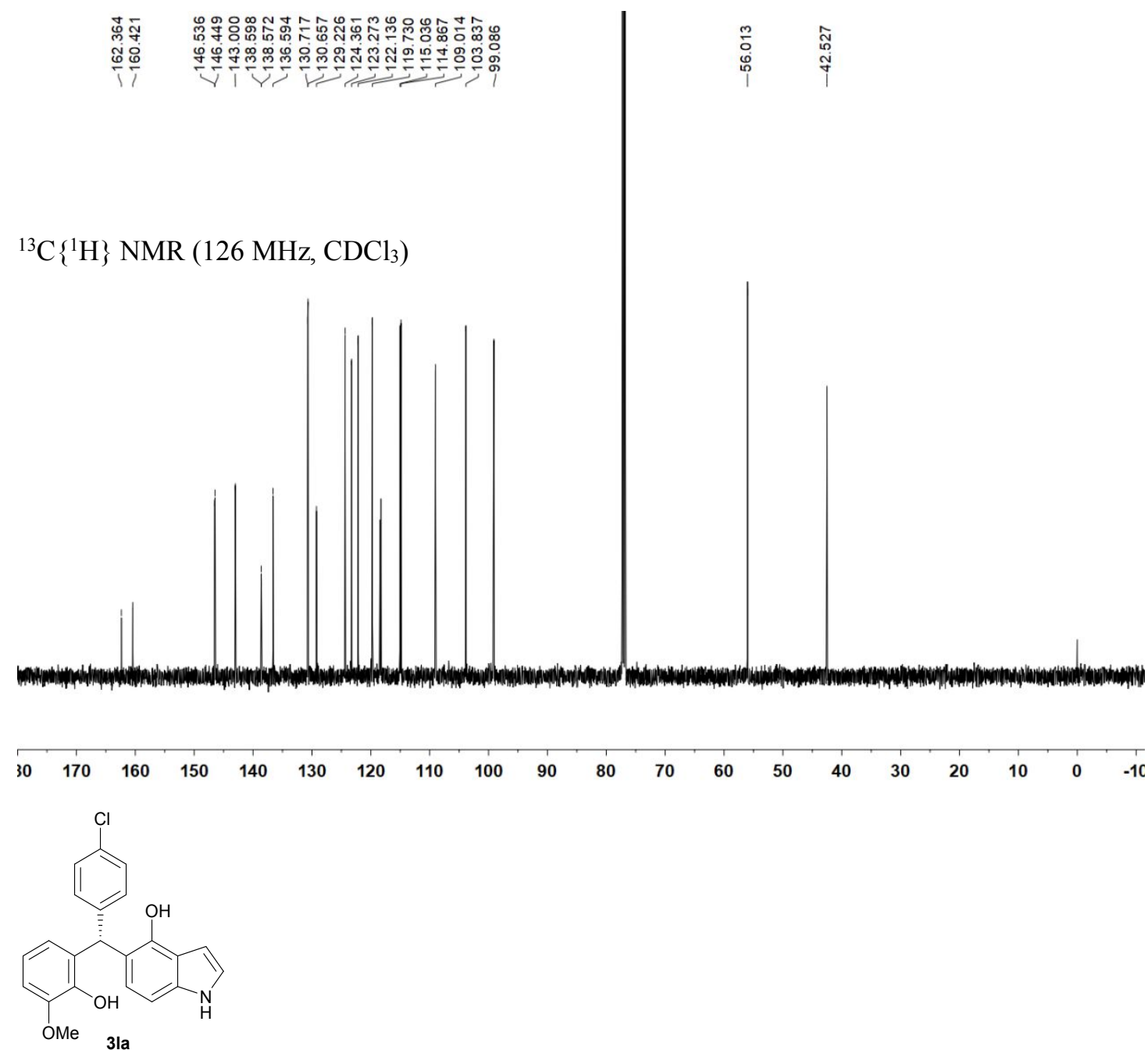

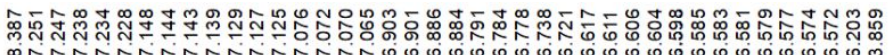

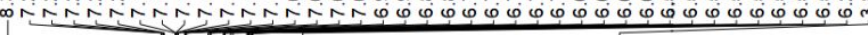

${ }^{1} \mathrm{H}$ NMR $\left(500 \mathrm{MHz}, \mathrm{CDCl}_{3}\right)$

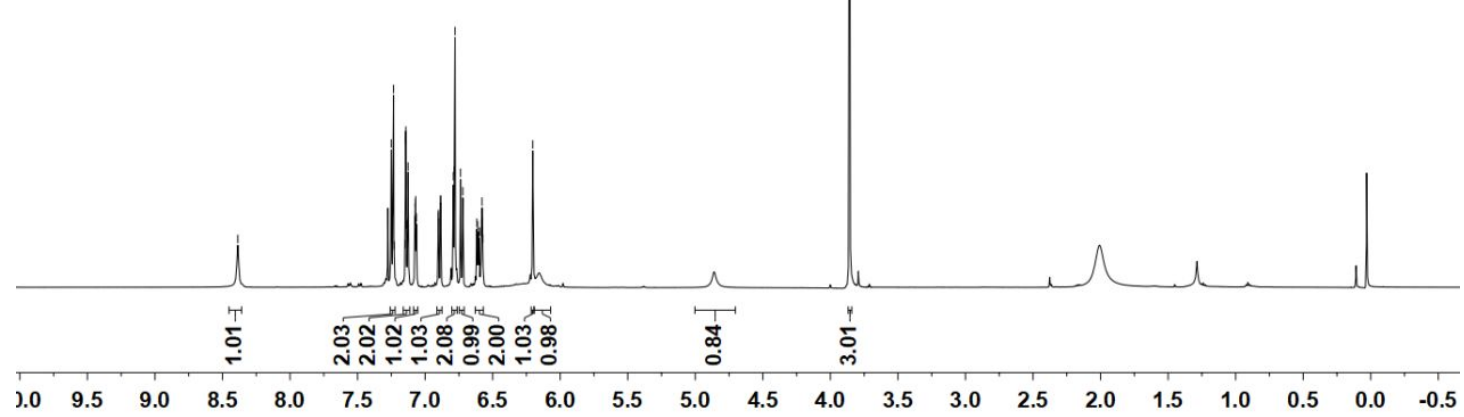




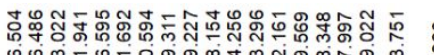

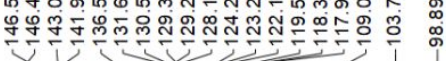

芯
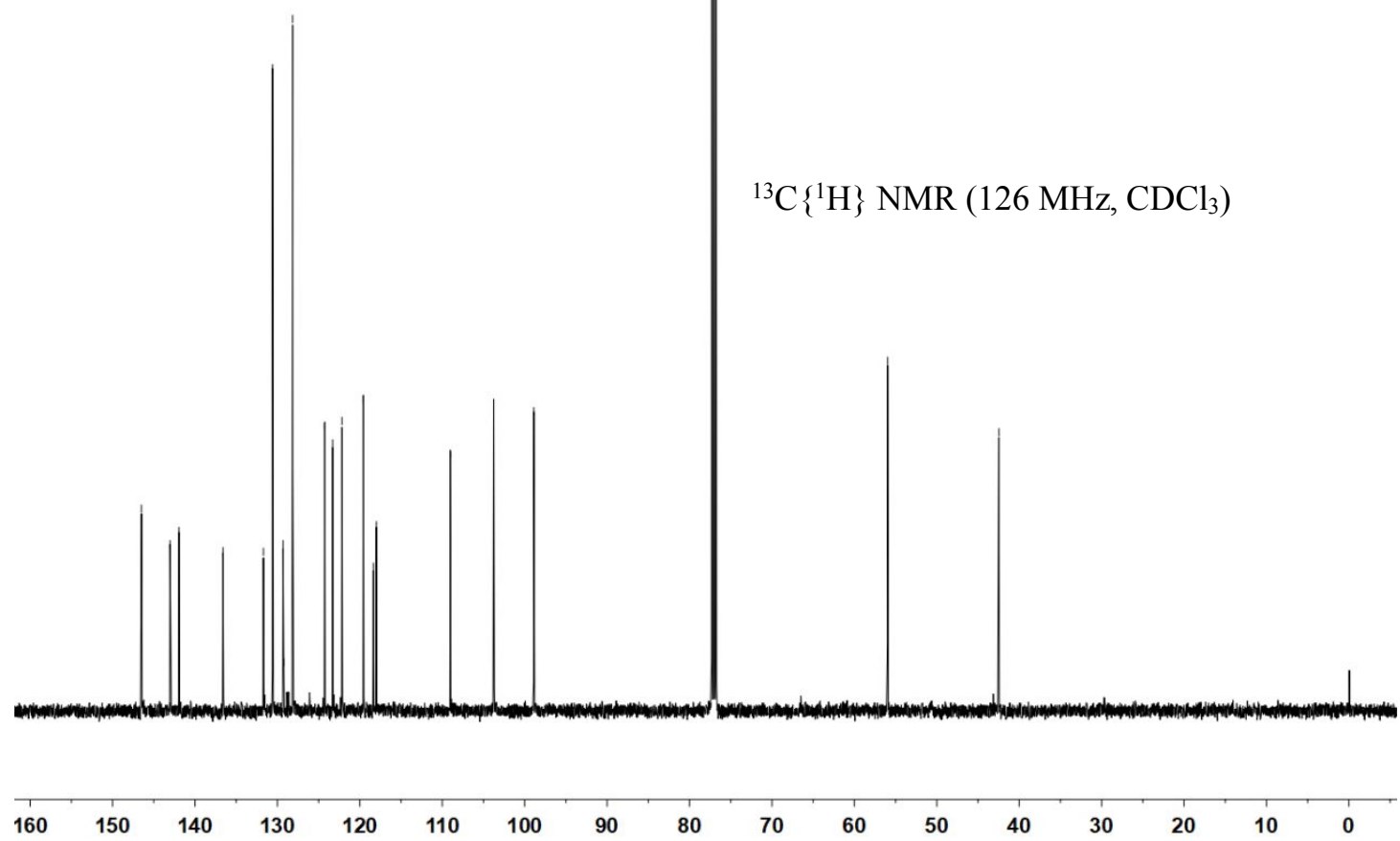<smiles>COc1ccccc1C(c1ccccc1C(F)(F)F)c1ccc2[nH]ccc2c1O</smiles>

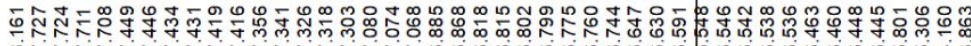

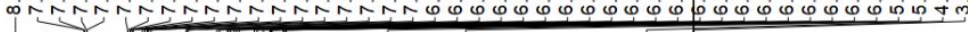

${ }^{1} \mathrm{H} \mathrm{NMR}\left(500 \mathrm{MHz}, \mathrm{CDCl}_{3}\right)$

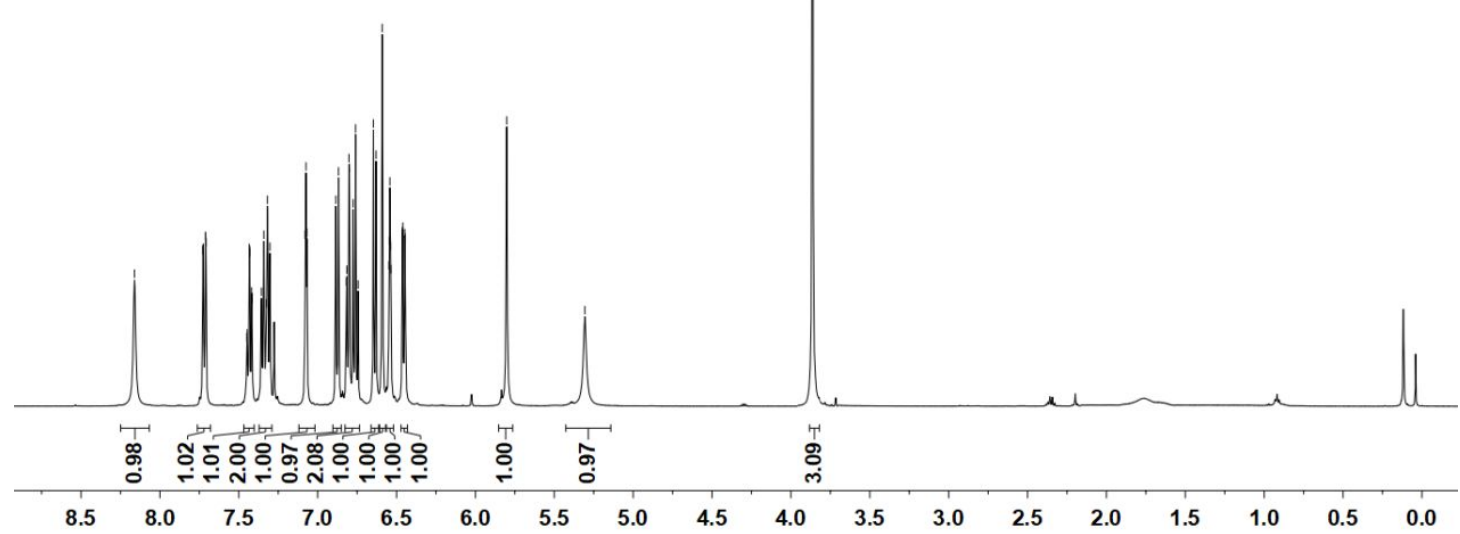



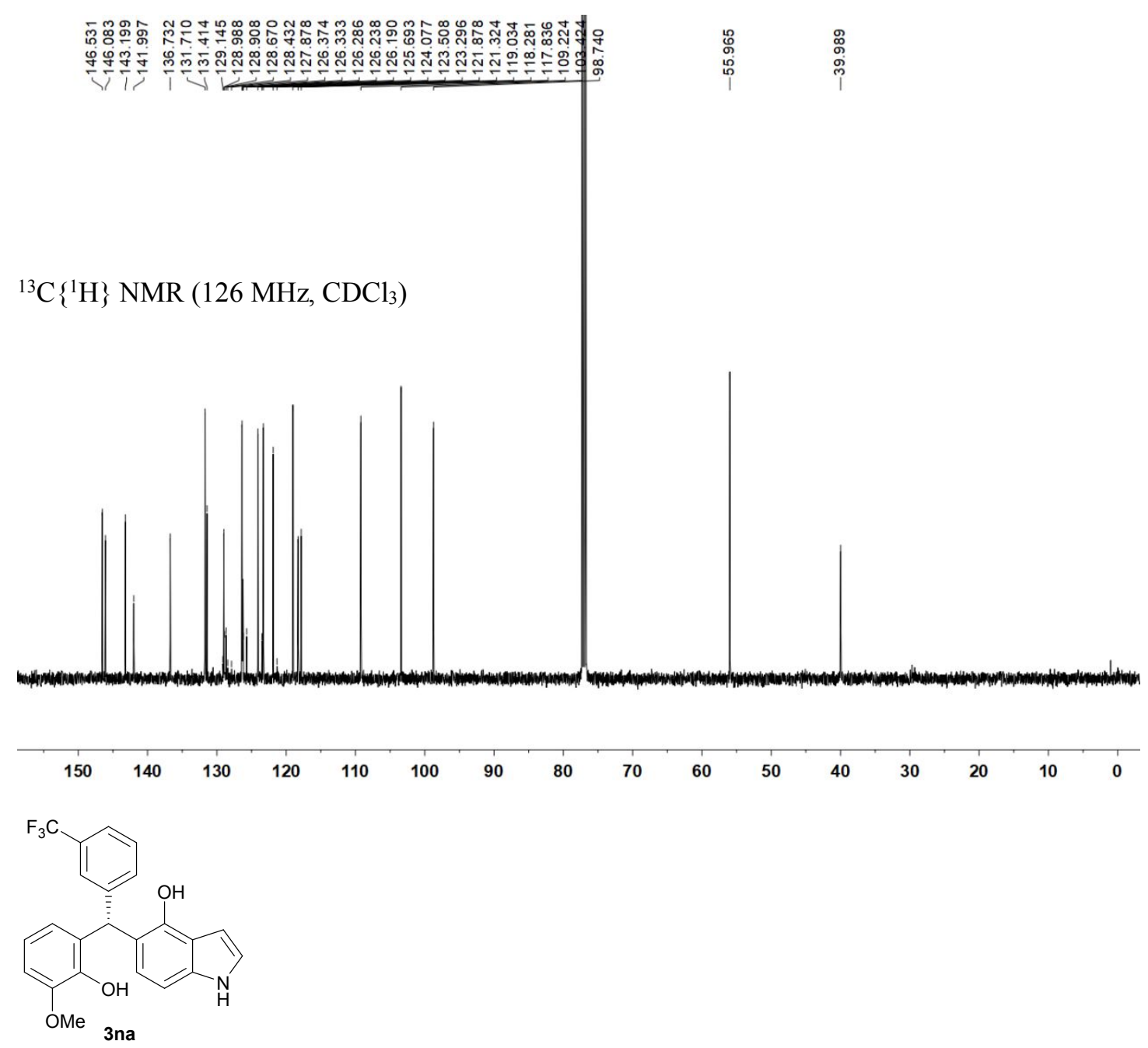

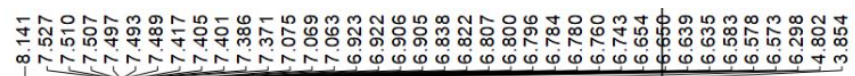

${ }^{1} \mathrm{H}$ NMR (500 MHz, $\mathrm{CDCl}_{3}$ )

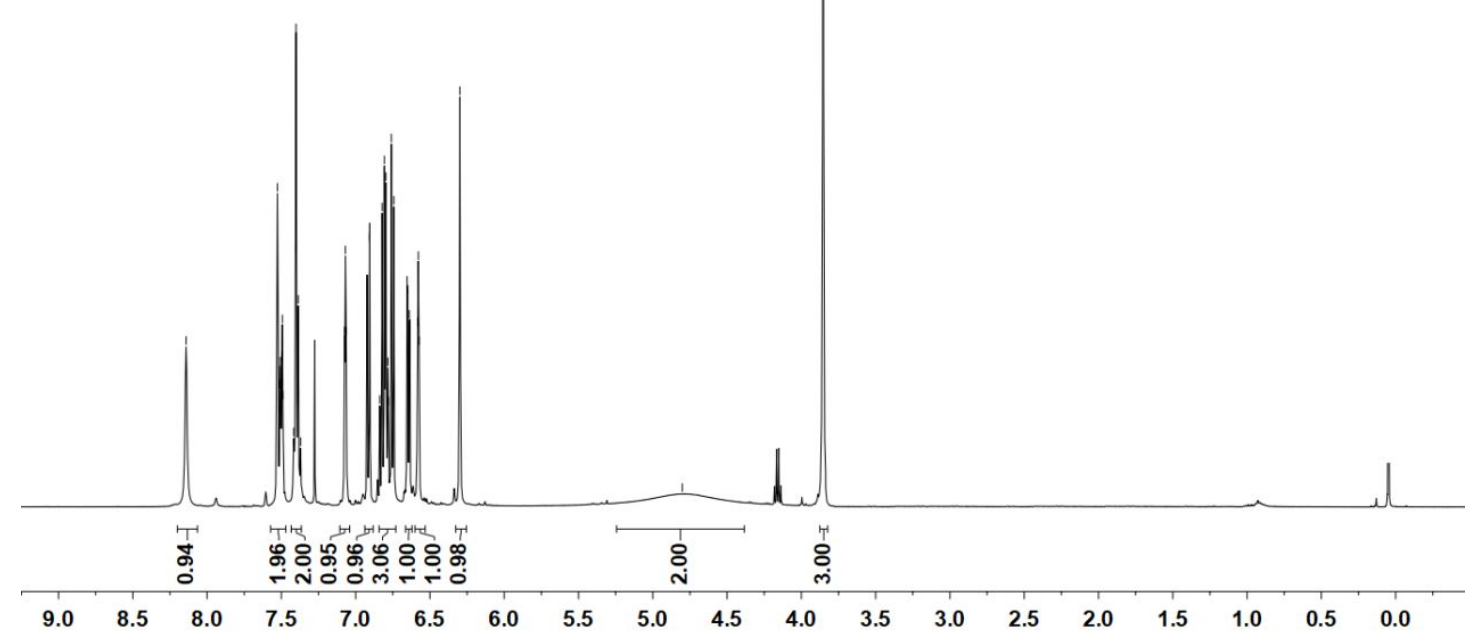


${ }^{13} \mathrm{C}\left\{{ }^{1} \mathrm{H}\right\}$ NMR $\left(126 \mathrm{MHz}, \mathrm{CDCl}_{3}\right)$
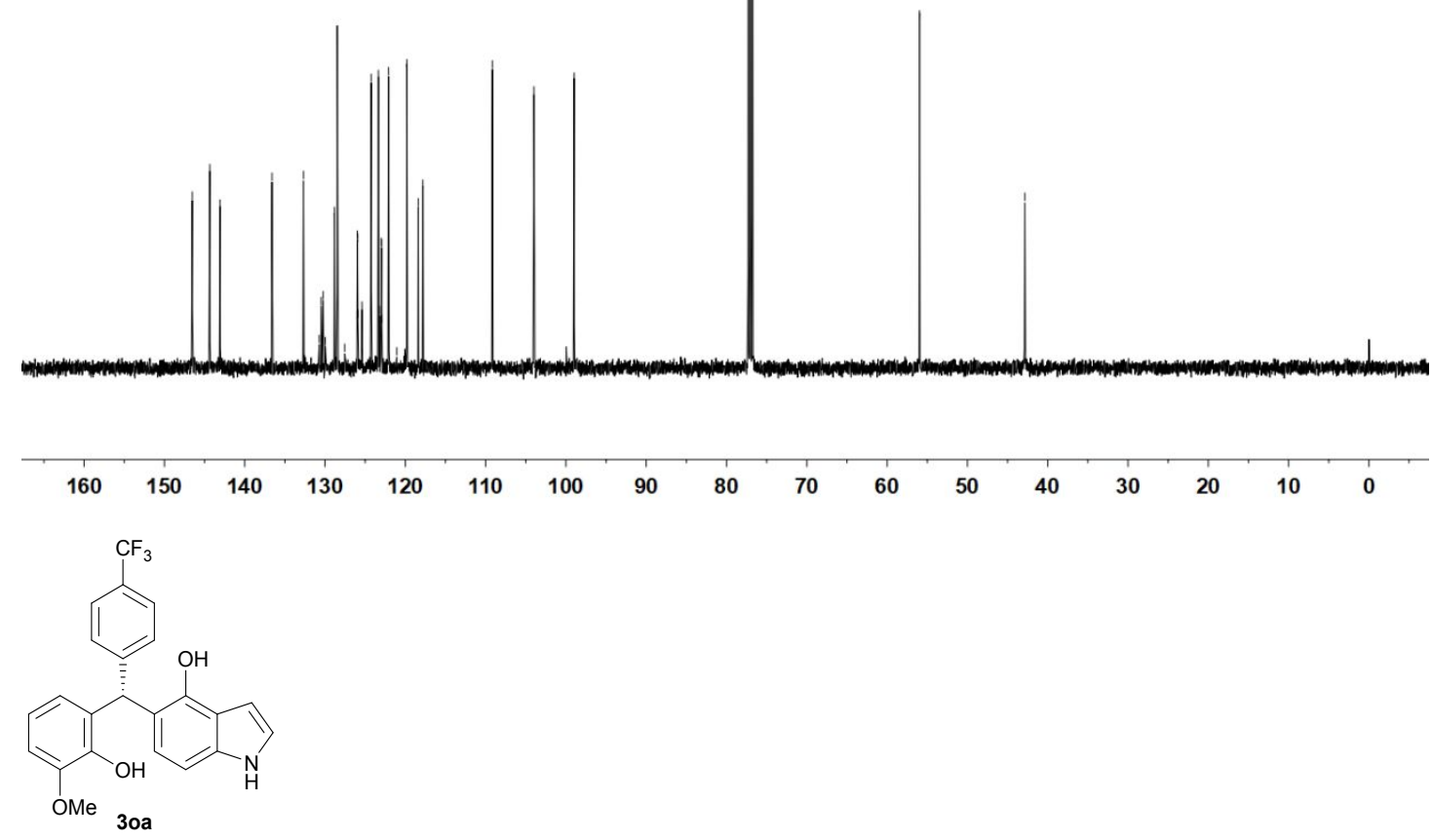

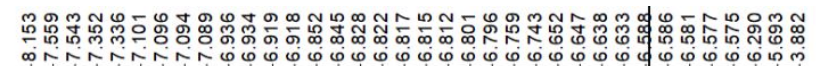

${ }^{1} \mathrm{H}$ NMR (500 MHz, $\mathrm{CDCl}_{3}$ )

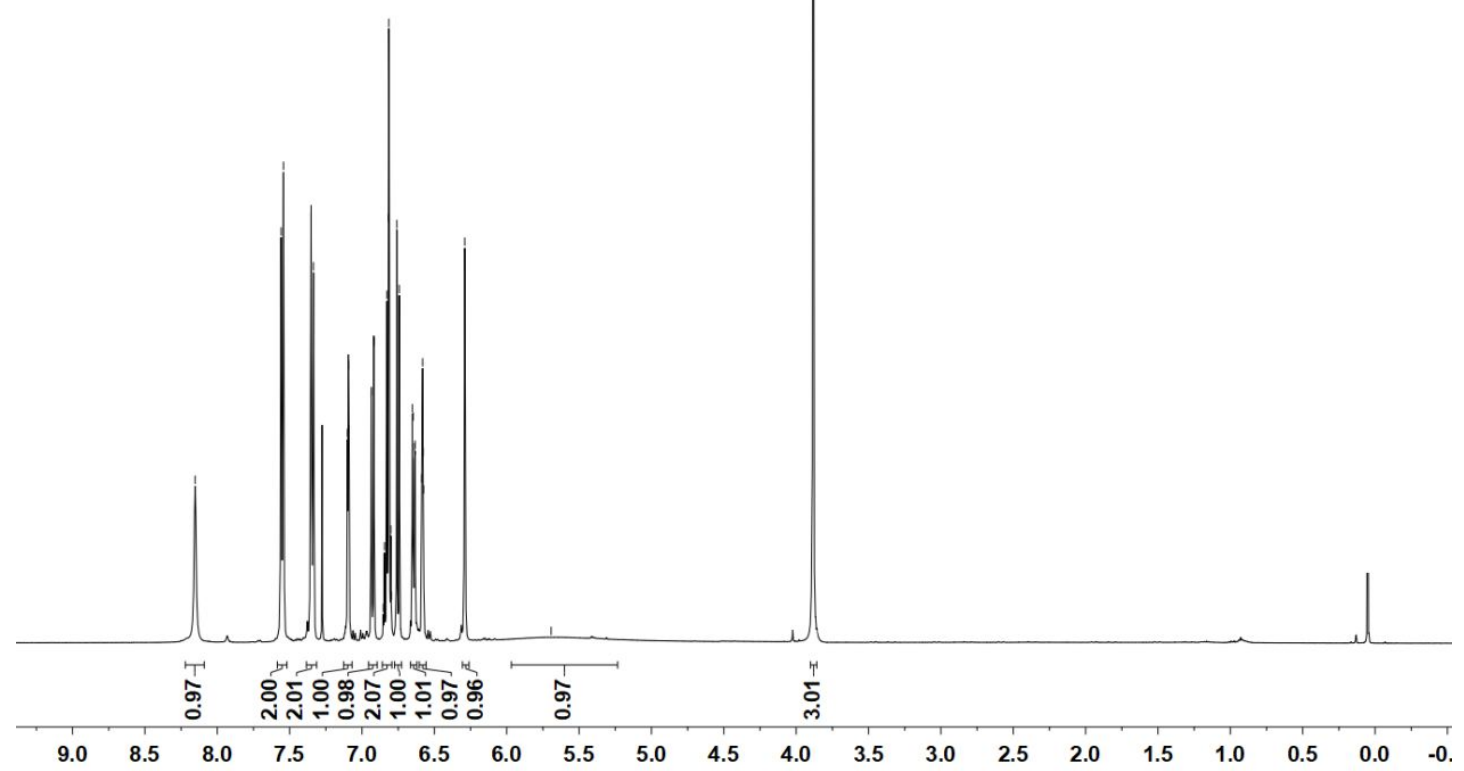


${ }^{13} \mathrm{C}\left\{{ }^{1} \mathrm{H}\right\}$ NMR $\left(126 \mathrm{MHz}, \mathrm{CDCl}_{3}\right)$
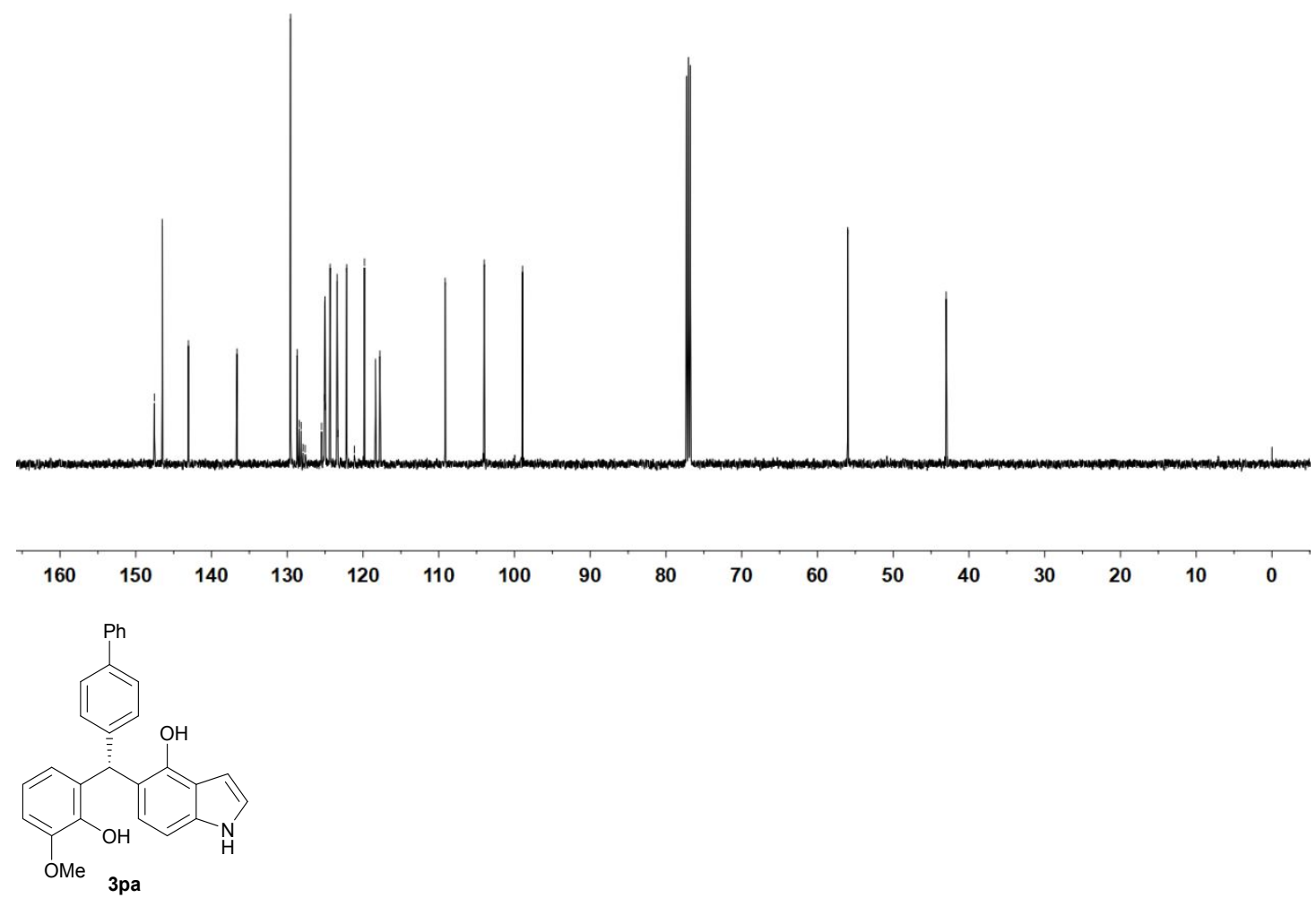

$\begin{aligned} & \\ & 0\end{aligned}$

${ }^{1} \mathrm{H}$ NMR $\left(600 \mathrm{MHz}, \mathrm{CDCl}_{3}\right)$

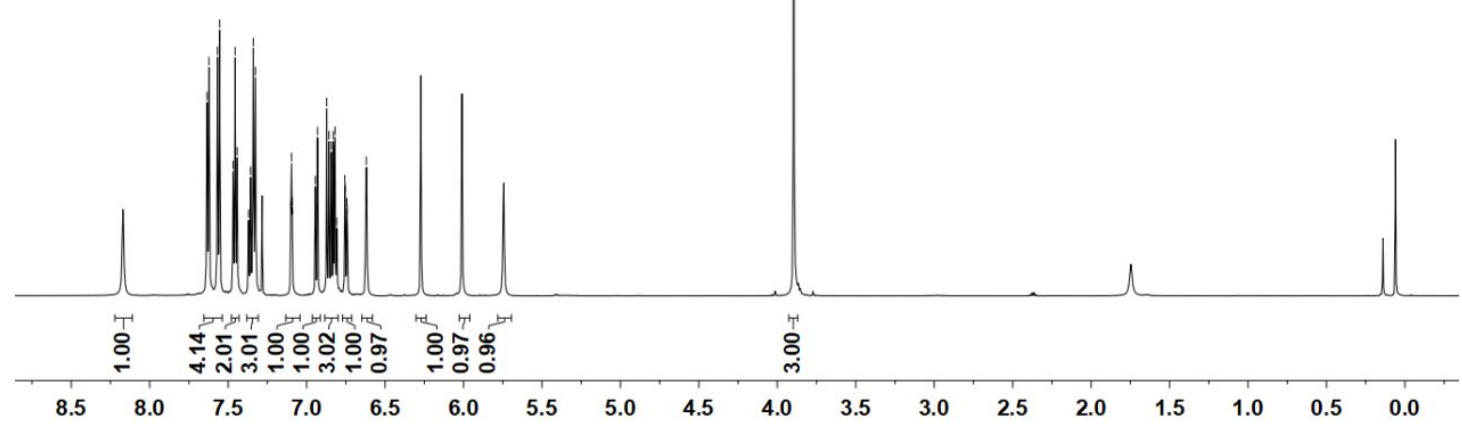


${ }^{13} \mathrm{C}\left\{{ }^{1} \mathrm{H}\right\} \mathrm{NMR}\left(151 \mathrm{MHz}, \mathrm{CDCl}_{3}\right)$
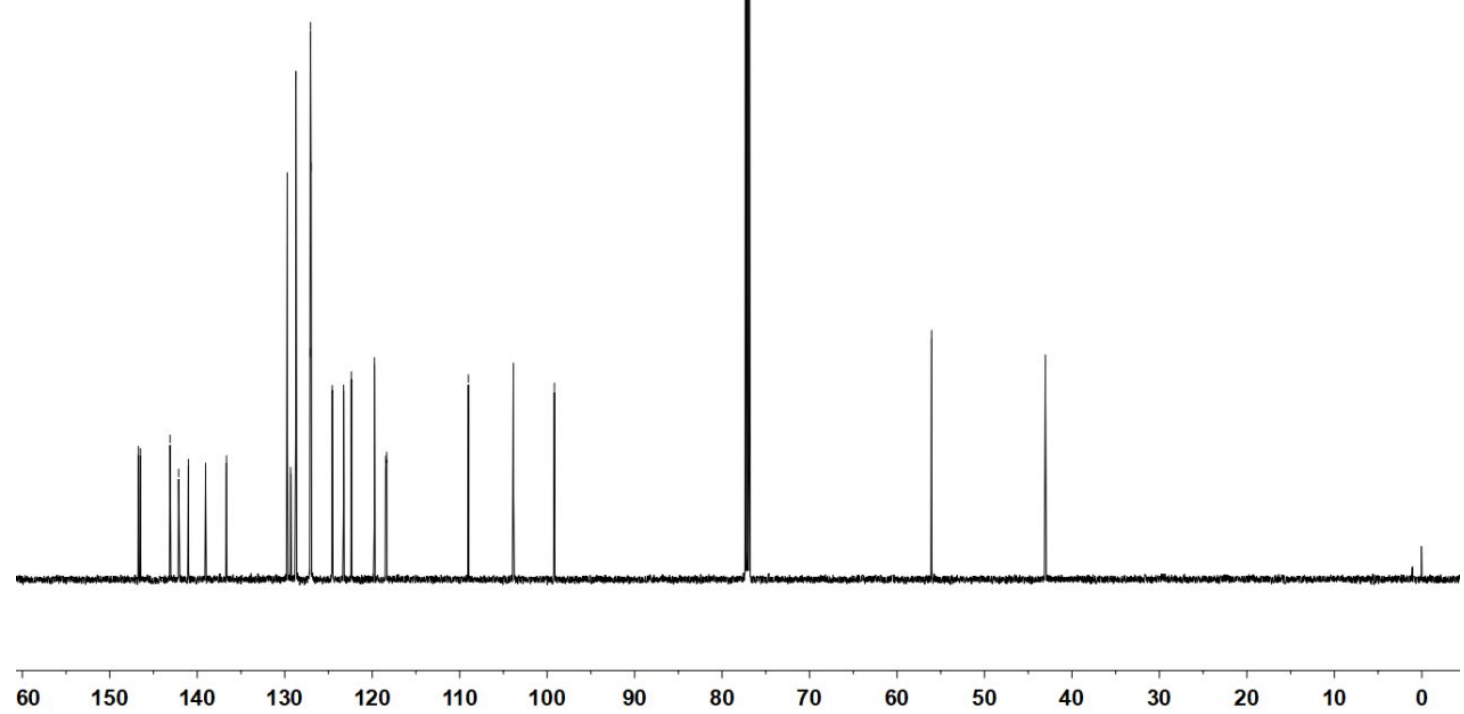<smiles>COc1cccc(C(c2ccc3[nH]ccc3c2O)c2cccc3ccccc23)c1O</smiles>

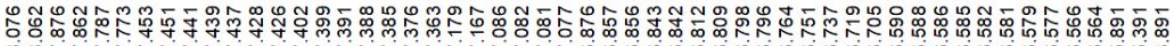

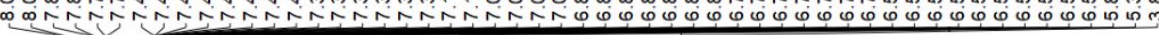

${ }^{1} \mathrm{H}$ NMR (600 MHz, $\mathrm{CDCl}_{3}$ )

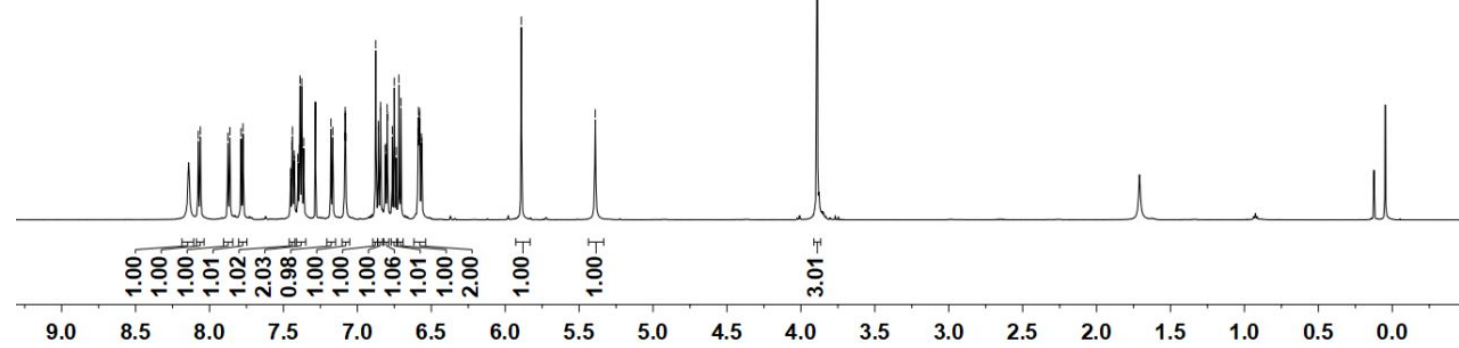


${ }^{13} \mathrm{C}\left\{{ }^{1} \mathrm{H}\right\}$ NMR (151 MHz, $\left.\mathrm{CDCl}_{3}\right)$
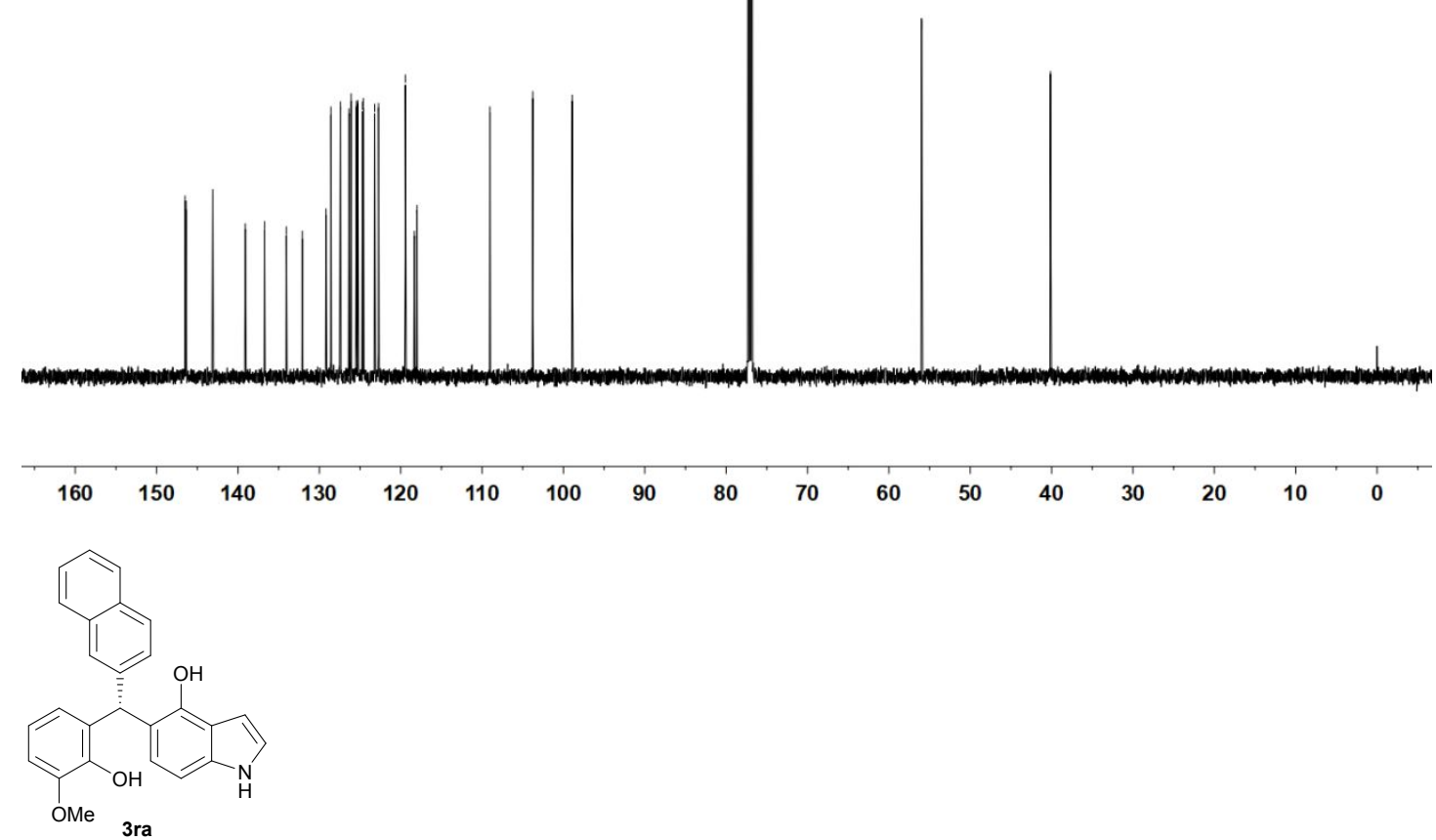

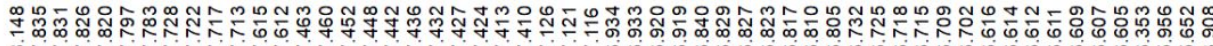

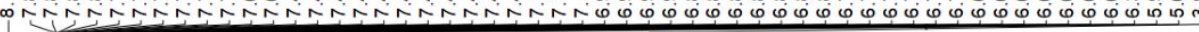

${ }^{1} \mathrm{H}$ NMR $\left(600 \mathrm{MHz}, \mathrm{CDCl}_{3}\right)$

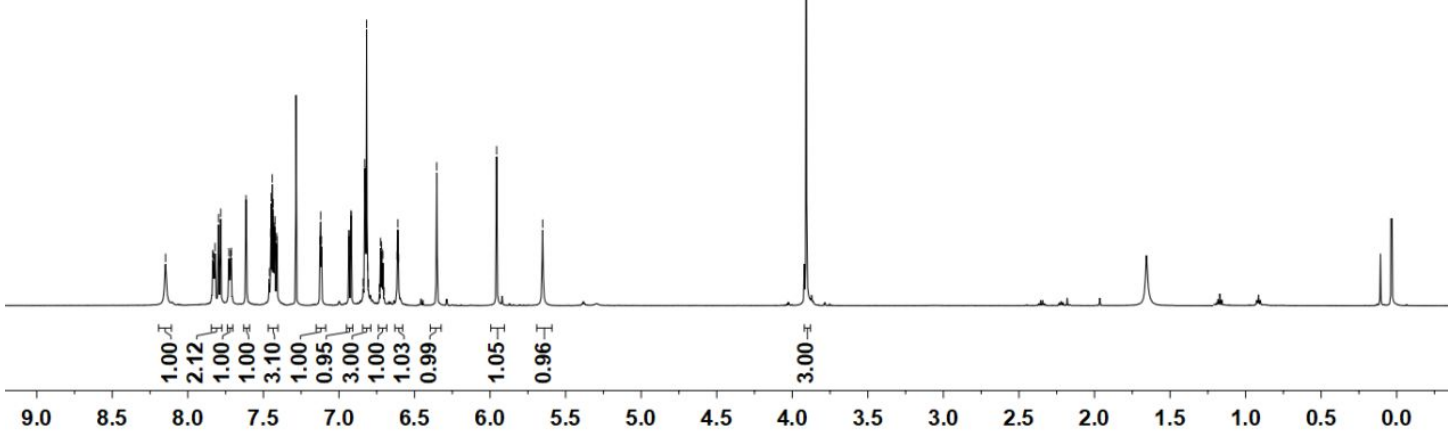



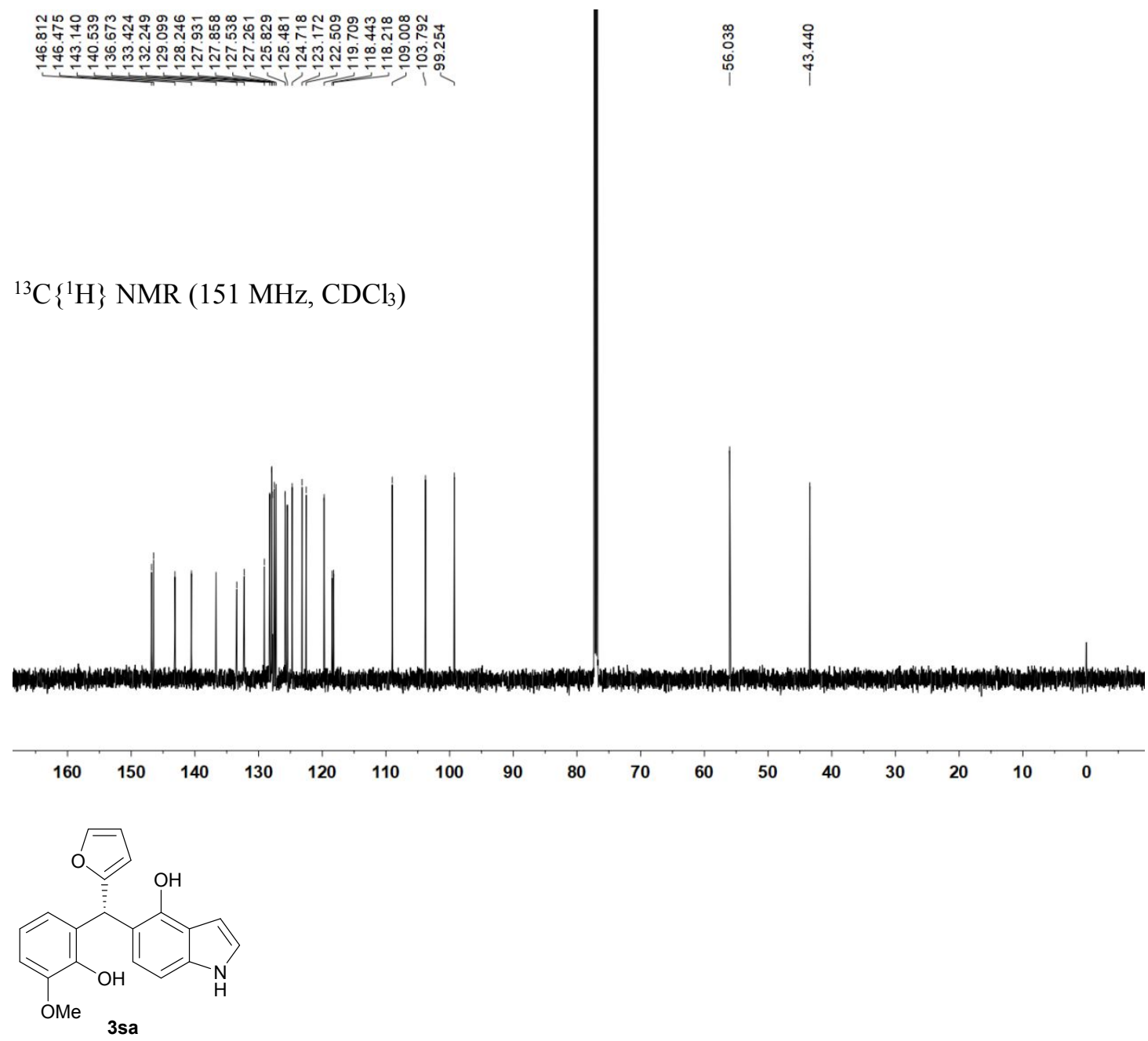

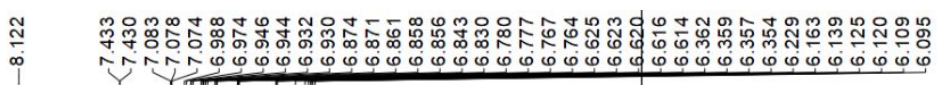

${ }^{1} \mathrm{H}$ NMR $\left(600 \mathrm{MHz}, \mathrm{CDCl}_{3}\right)$

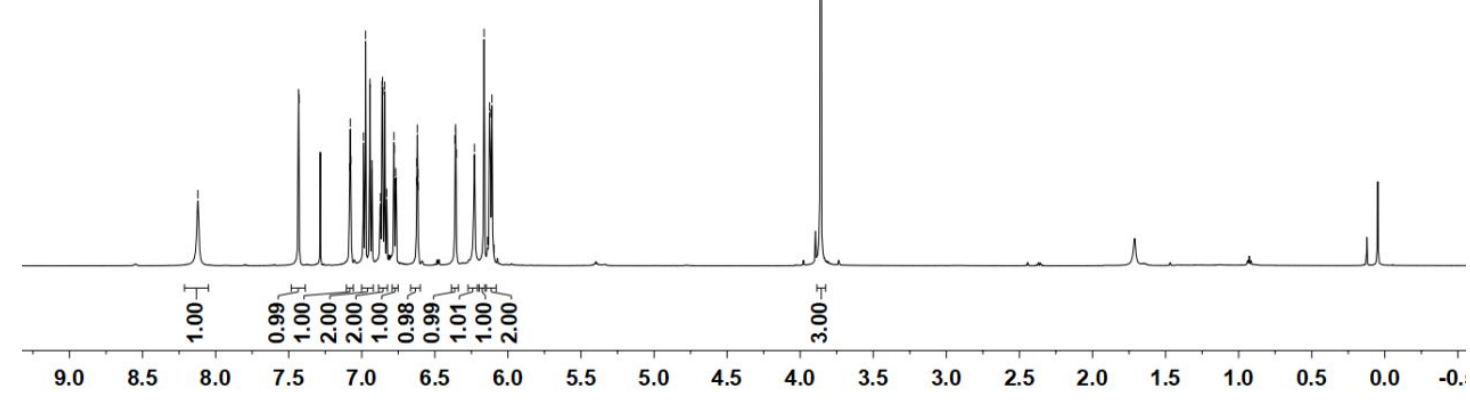



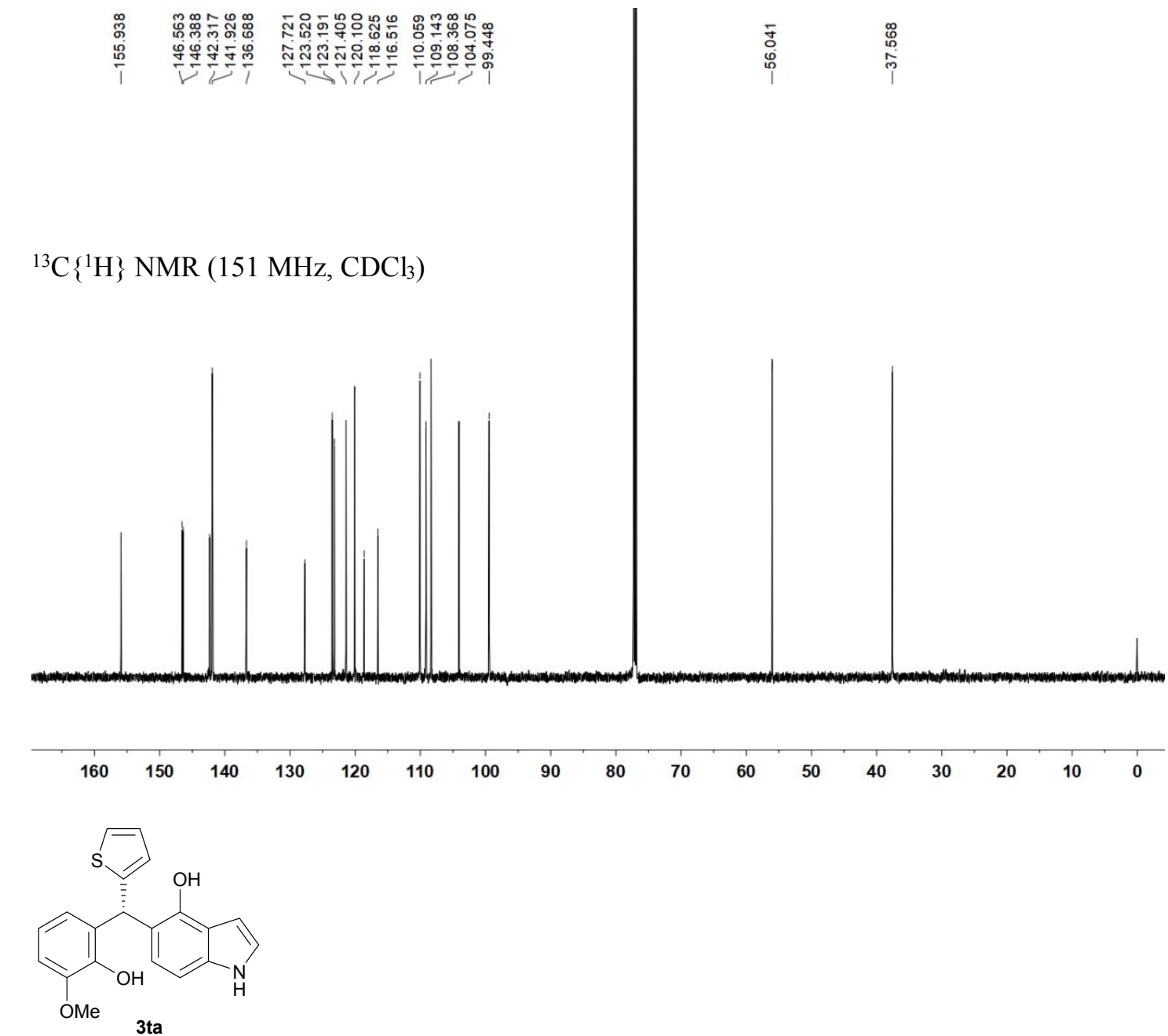

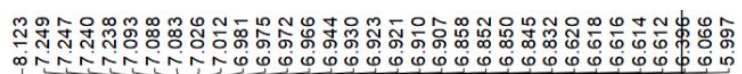

${ }^{1} \mathrm{H}$ NMR $\left(600 \mathrm{MHz}, \mathrm{CDCl}_{3}\right)$

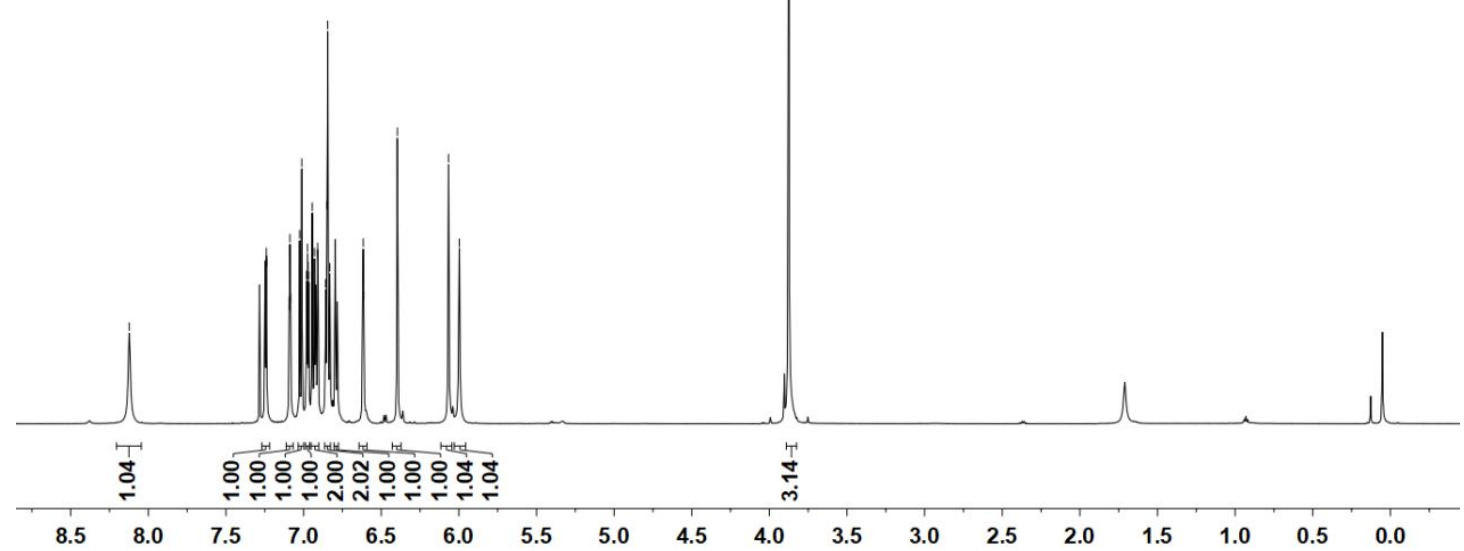


${ }^{13} \mathrm{C}\left\{{ }^{1} \mathrm{H}\right\}$ NMR $\left(151 \mathrm{MHz}, \mathrm{CDCl}_{3}\right)$
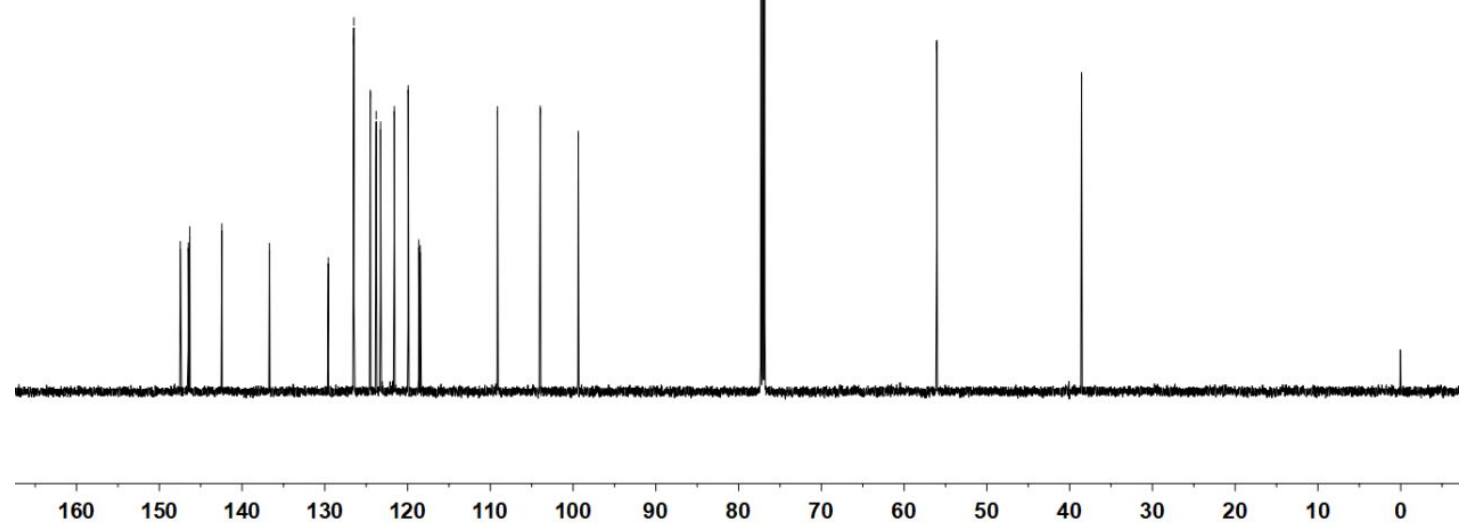<smiles>COc1cccc(C(c2ccccc2)c2c(O)ccc3[nH]ccc23)c1O</smiles>

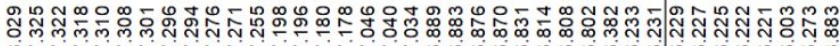

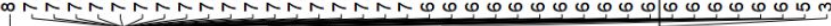

${ }^{1} \mathrm{H} \mathrm{NMR}\left(500 \mathrm{MHz}, \mathrm{CDCl}_{3}\right)$

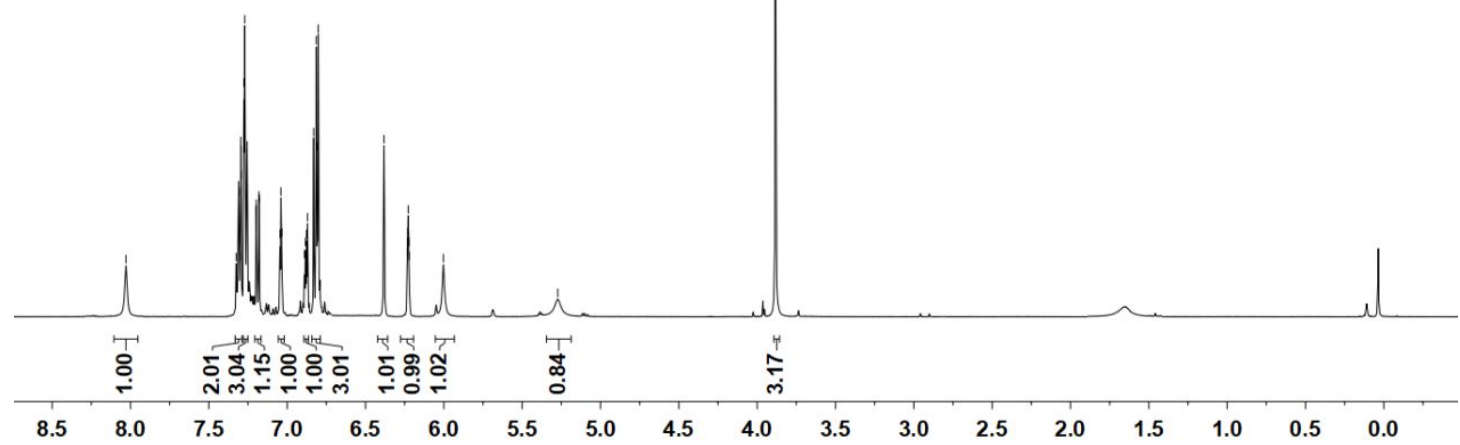


${ }^{13} \mathrm{C}\left\{{ }^{1} \mathrm{H}\right\} \operatorname{NMR}\left(126 \mathrm{MHz}, \mathrm{CDCl}_{3}\right)$
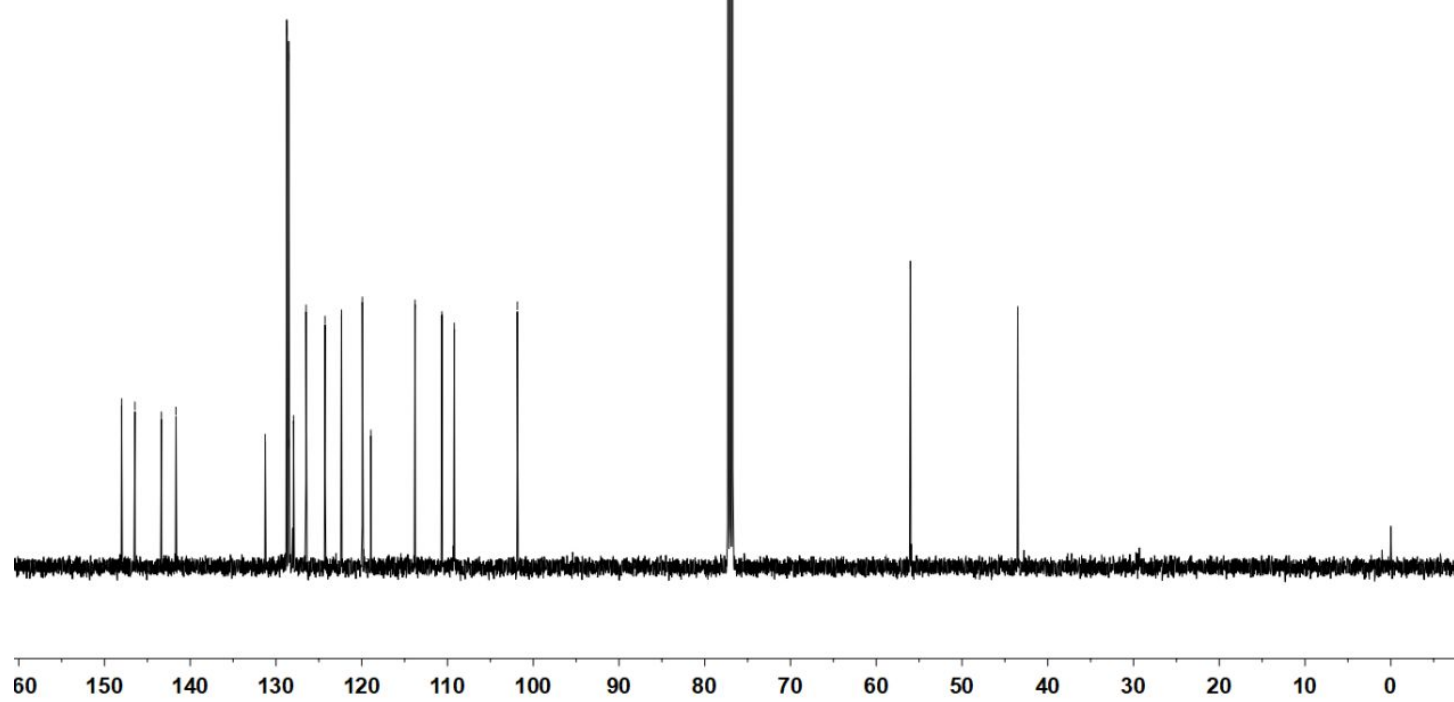<smiles></smiles>

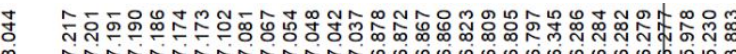

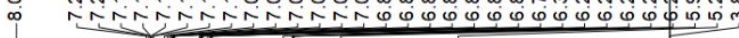

$\stackrel{\text { 品 }}{\stackrel{\sim}{\sim}}$

${ }^{1} \mathrm{H}$ NMR $\left(500 \mathrm{MHz}, \mathrm{CDCl}_{3}\right)$

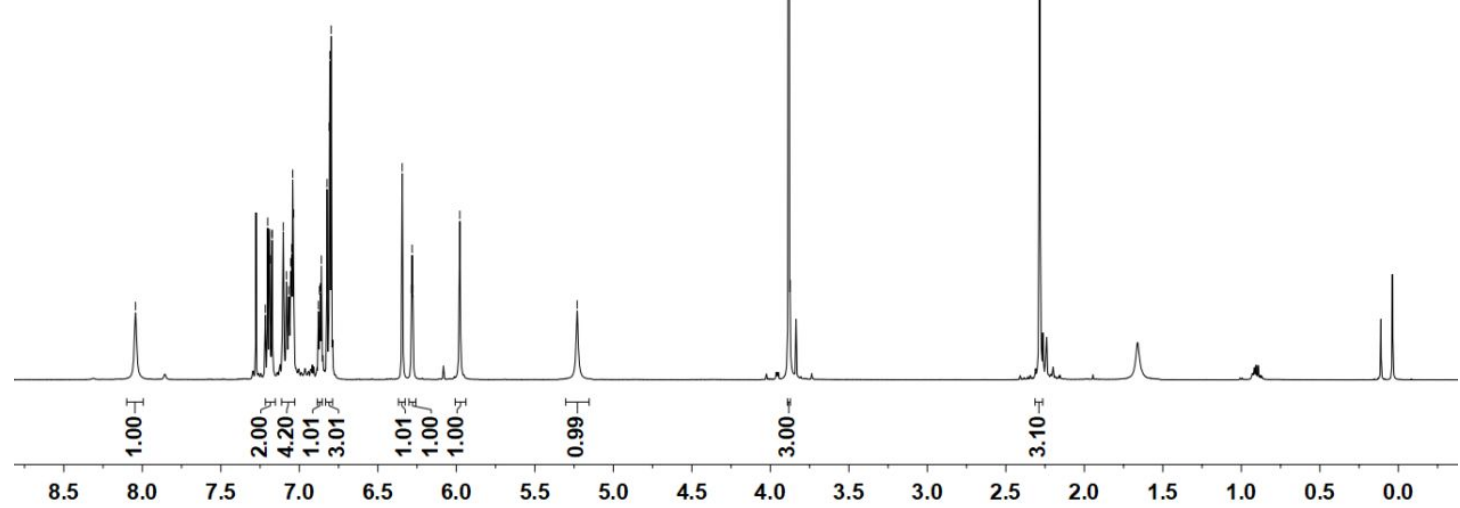


${ }^{13} \mathrm{C}\left\{{ }^{1} \mathrm{H}\right\}$ NMR (126 MHz, $\left.\mathrm{CDCl}_{3}\right)$
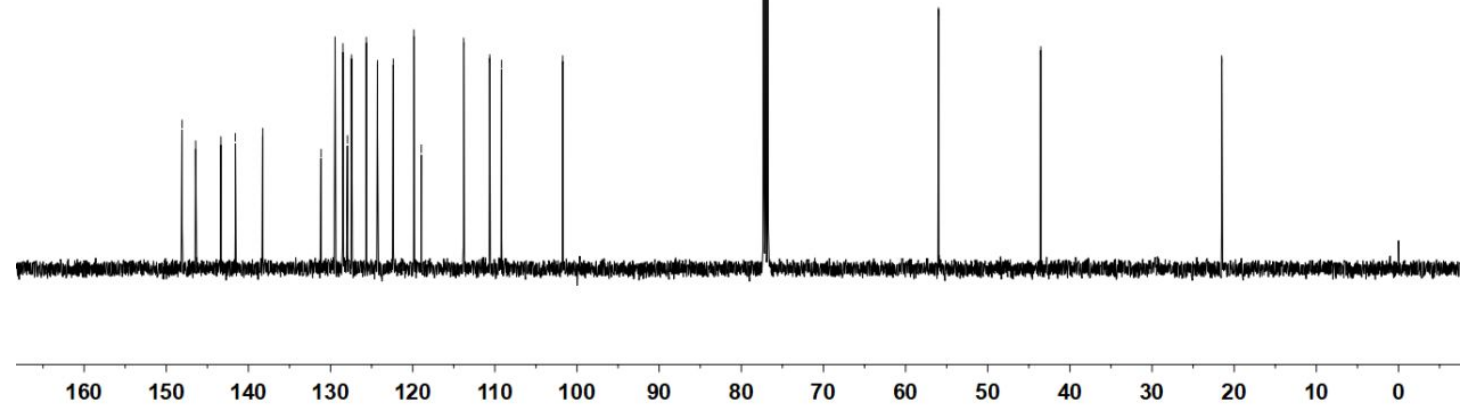<smiles>COc1cccc(O)c1C(c1ccc(Cl)cc1)c1c(O)ccc2[nH]ccc12</smiles>

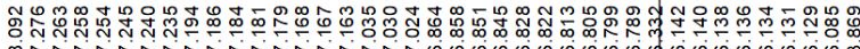

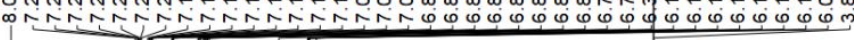

${ }^{1} \mathrm{H}$ NMR $\left(500 \mathrm{MHz}, \mathrm{CDCl}_{3}\right)$

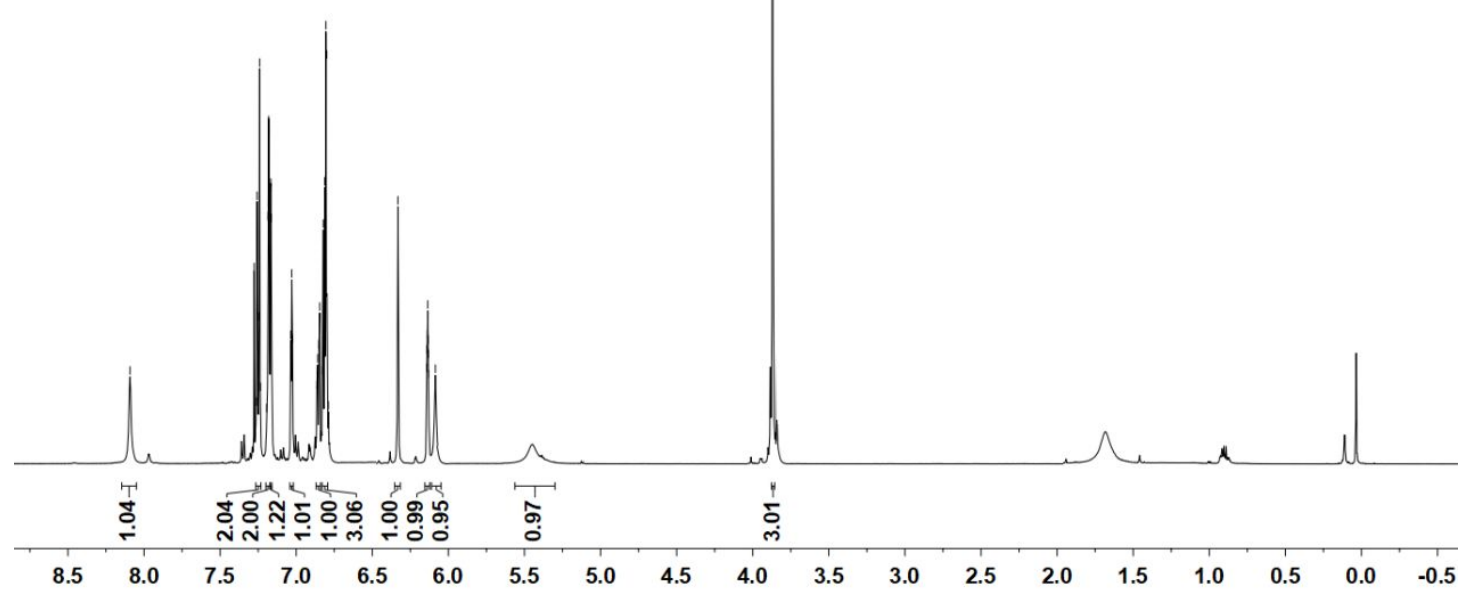




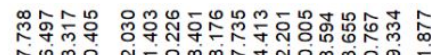

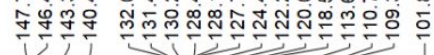

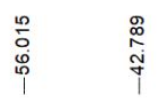

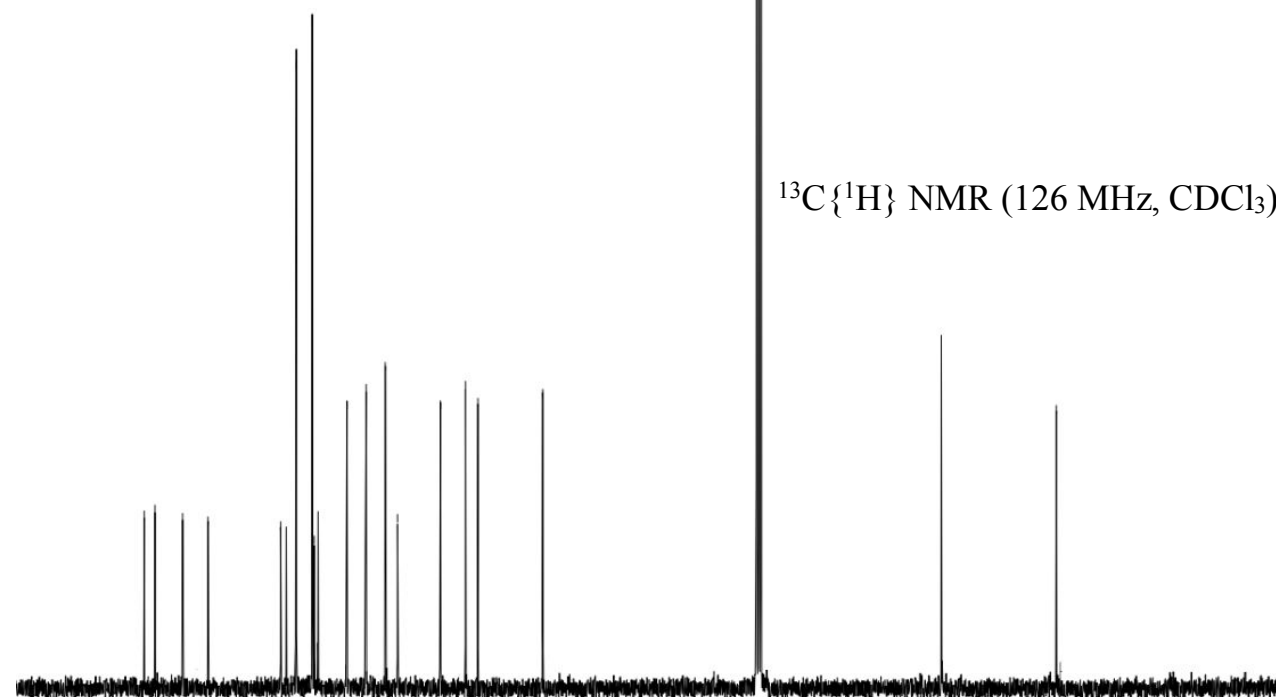

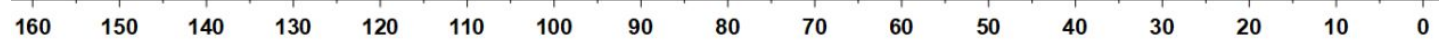<smiles>COc1cccc(C(c2ccccc2)c2ccc3cc[nH]c3c2O)c1O</smiles>

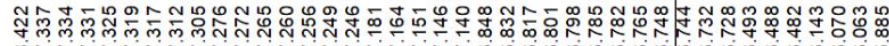

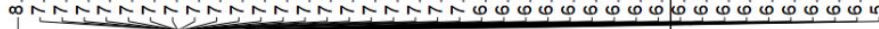

${ }^{1} \mathrm{H}$ NMR $\left(500 \mathrm{MHz}, \mathrm{CDCl}_{3}\right)$

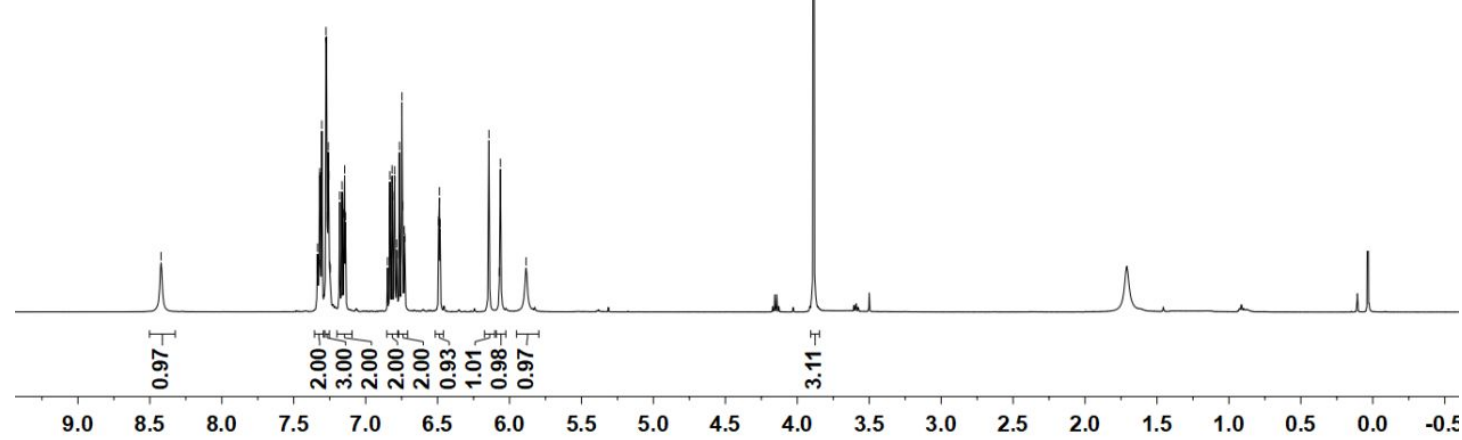




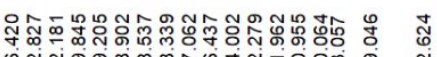

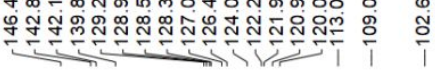

${ }^{13} \mathrm{C}\left\{{ }^{1} \mathrm{H}\right\}$ NMR $\left(126 \mathrm{MHz}, \mathrm{CDCl}_{3}\right)$
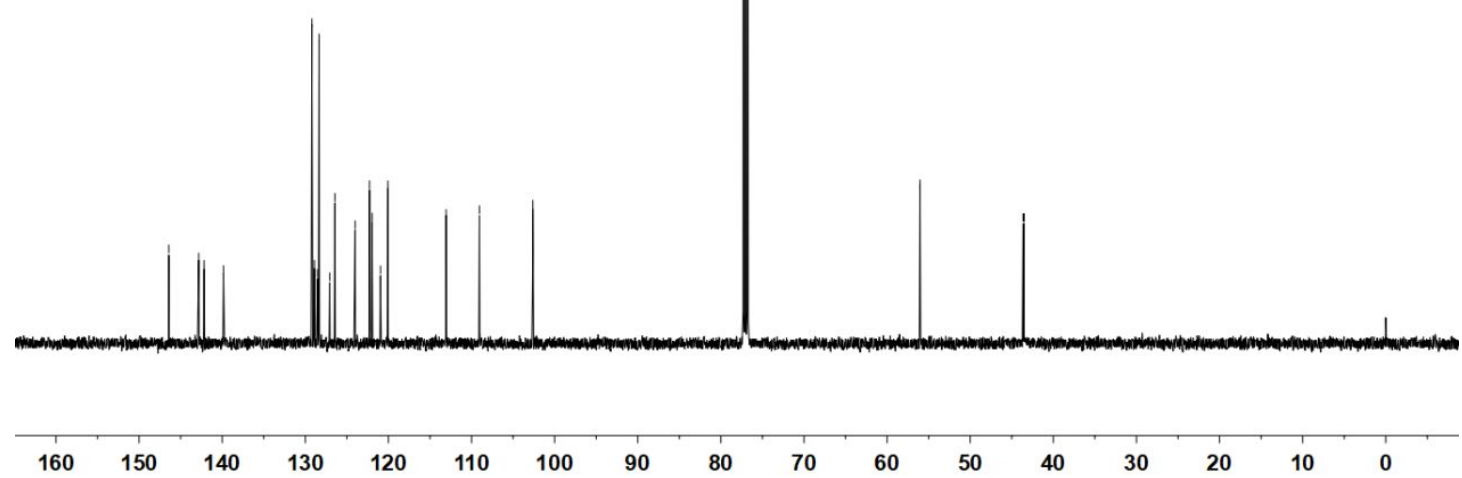<smiles>CCc1ccccc1C(c1cccc(OC)c1O)c1ccc2cc[nH]c2c1O</smiles>

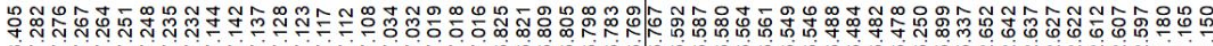

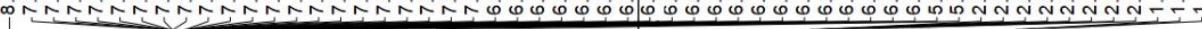

${ }^{1} \mathrm{H}$ NMR $\left(500 \mathrm{MHz}, \mathrm{CDCl}_{3}\right)$

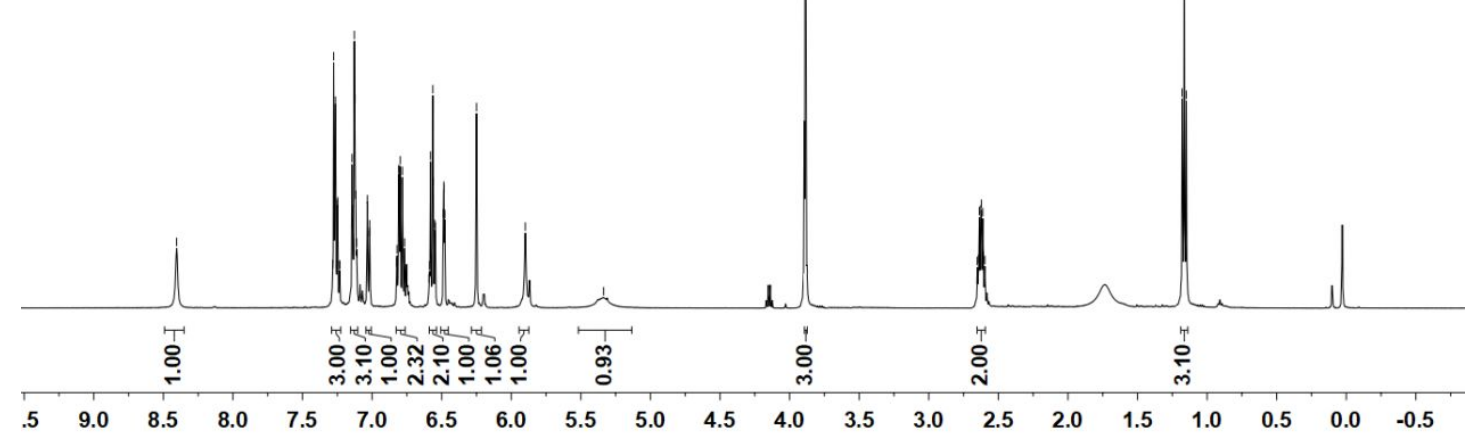


${ }^{13} \mathrm{C}\left\{{ }^{1} \mathrm{H}\right\}$ NMR $\left(126 \mathrm{MHz}, \mathrm{CDCl}_{3}\right)$

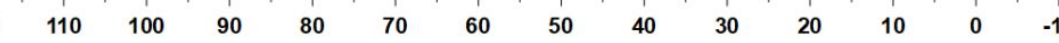<smiles>Oc1cccc(C(c2ccccc2C(F)(F)F)c2ccc3cc[nH]c3c2O)c1O</smiles>

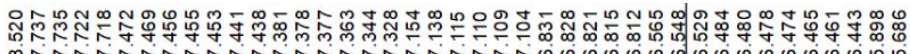

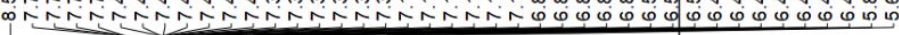

${ }^{1} \mathrm{H}$ NMR $\left(500 \mathrm{MHz}, \mathrm{CDCl}_{3}\right)$

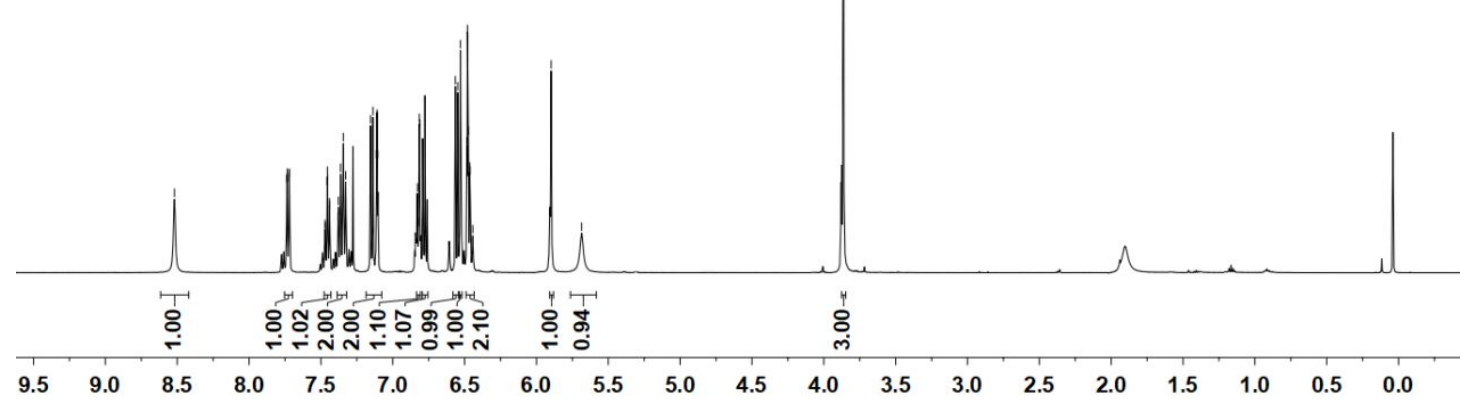


${ }^{13} \mathrm{C}\left\{{ }^{1} \mathrm{H}\right\} \mathrm{NMR}\left(126 \mathrm{MHz}, \mathrm{CDCl}_{3}\right)$

$\begin{array}{lllllllllllllllllllllllllllll}160 & 155 & 150 & 145 & 140 & 135 & 130 & 125 & 120 & 115 & 110 & 105 & 100 & 95 & 90 & 85 & 80 & 75 & 70 & 65 & 60 & 55 & 50 & 45 & 40 & 35 & 30\end{array}$<smiles>COc1cccc([C@H](c2ccccc2)c2ccc3[nH]c4ccccc4c3c2O)c1O</smiles>

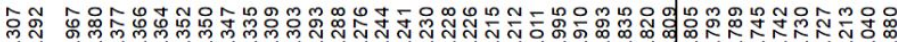

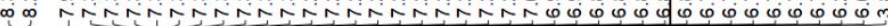

${ }^{1} \mathrm{H}$ NMR (500 MHz, $\mathrm{CDCl}_{3}$ )

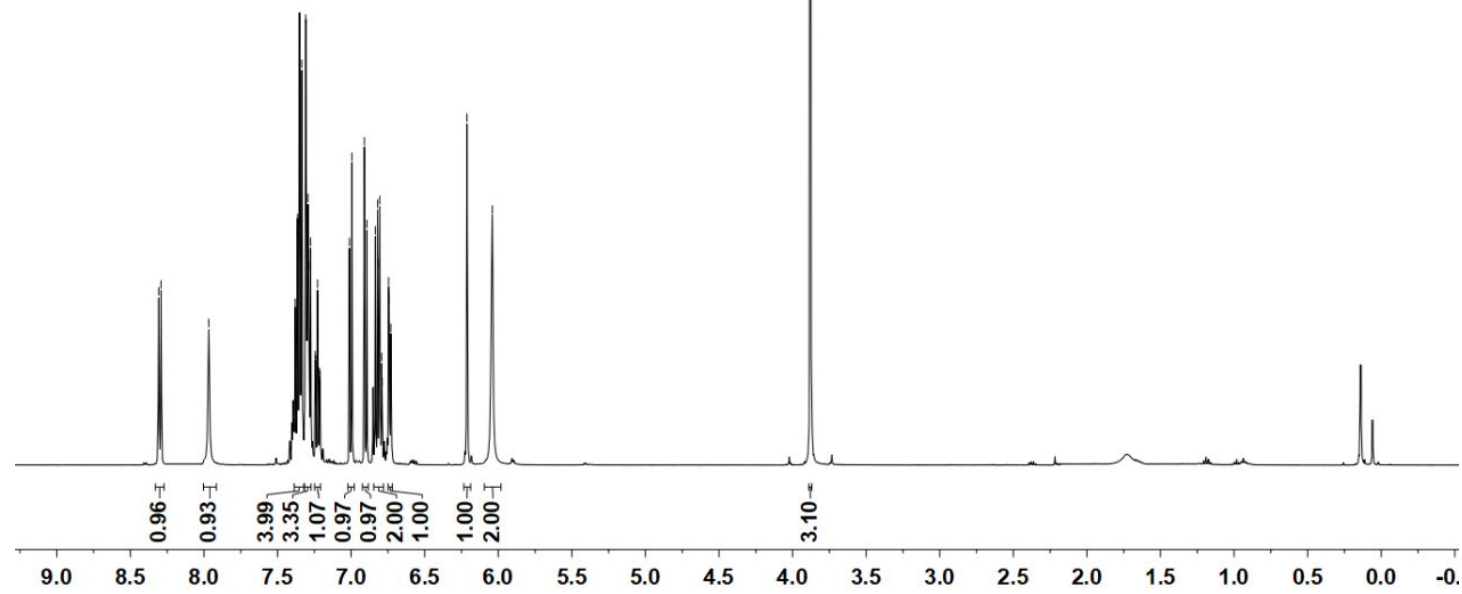




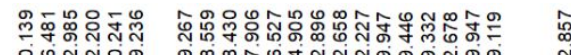

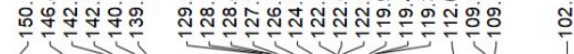

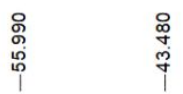

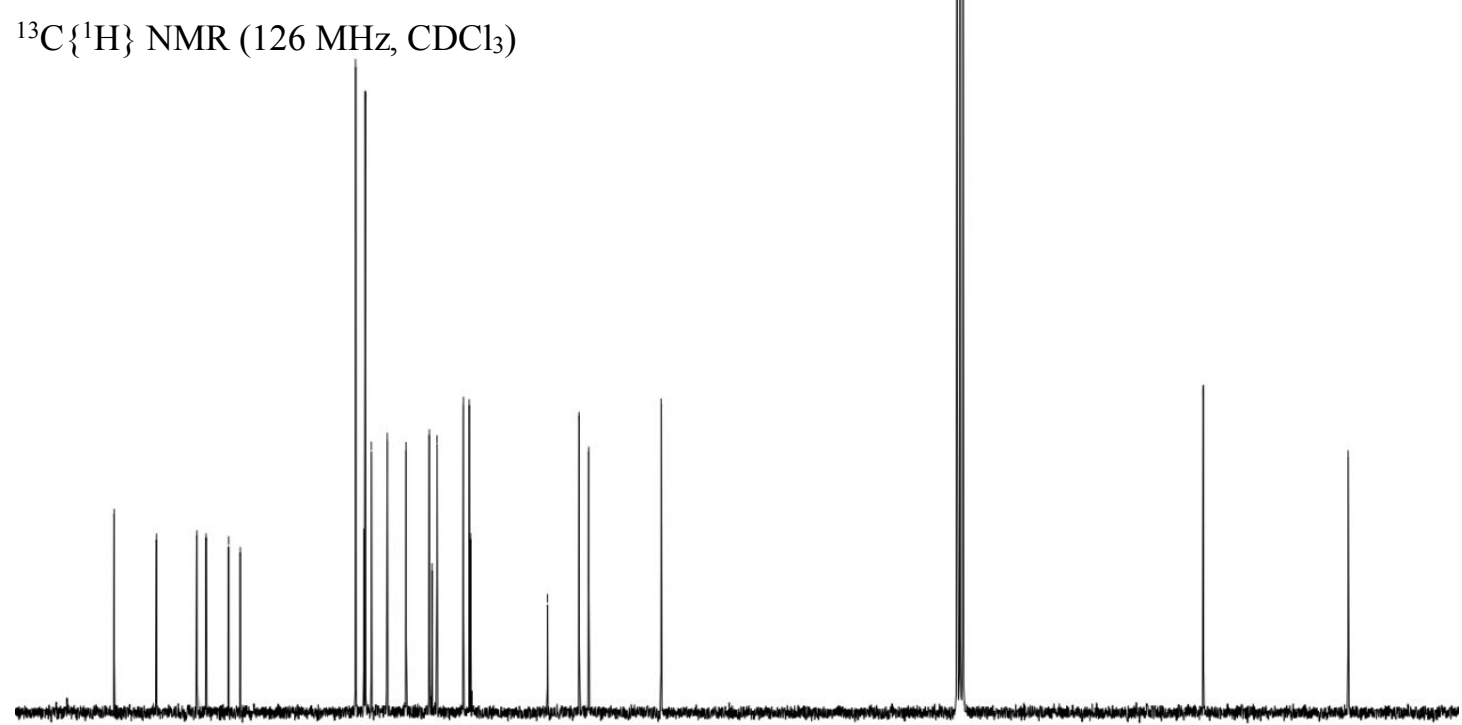

$\begin{array}{lllllllllllllllllllllllll}155 & 150 & 145 & 140 & 135 & 130 & 125 & 120 & 115 & 110 & 105 & 100 & 95 & 90 & 85 & 80 & 75 & 70 & 65 & 60 & 55 & 50 & 45 & 40 & 35\end{array}$<smiles>COc1cccc(C(c2ccccc2)c2ccc3[nH]ccc3c2O)c1</smiles>

兽议

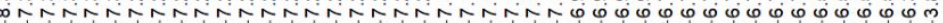

${ }^{1} \mathrm{H}$ NMR $\left(600 \mathrm{MHz}, \mathrm{CDCl}_{3}\right)$

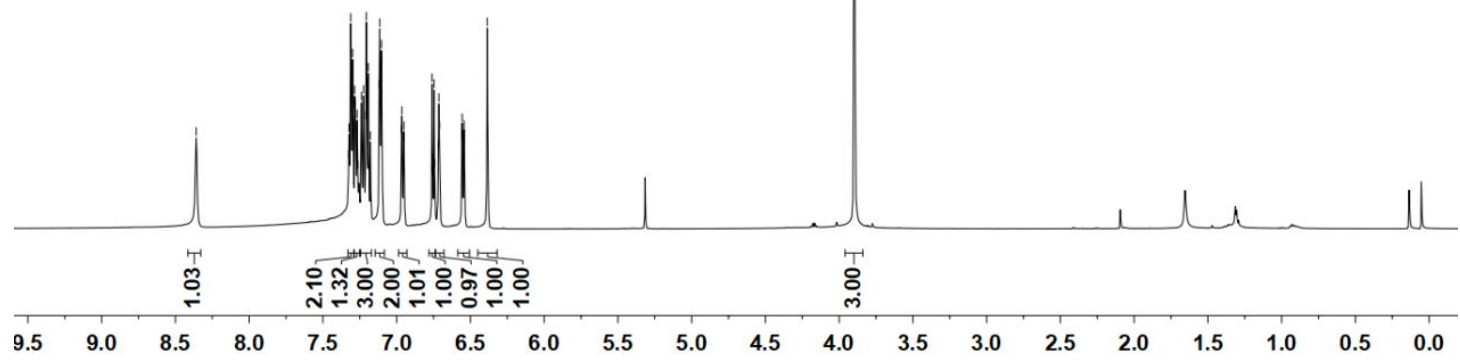


${ }^{13} \mathrm{C}\left\{{ }^{1} \mathrm{H}\right\}$ NMR $\left(151 \mathrm{MHz}, \mathrm{CDCl}_{3}\right)$

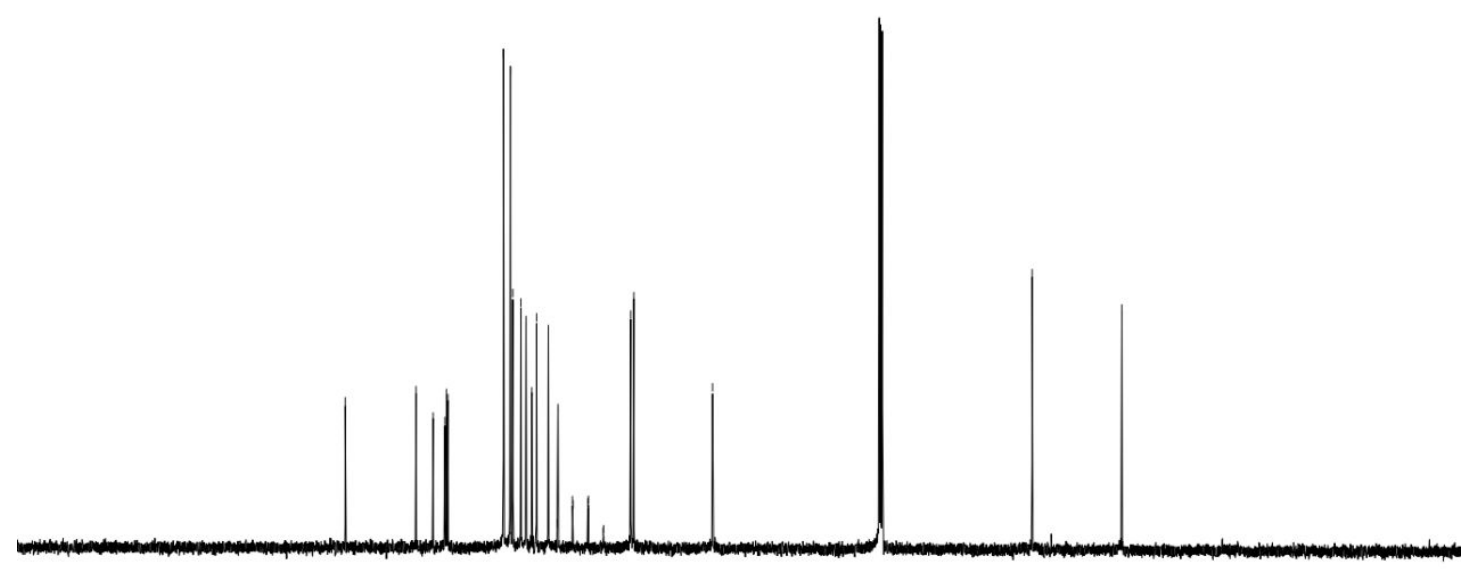

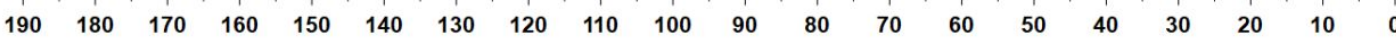




\section{X-Ray structure of compound 4}<smiles>COc1cccc(C(c2ccccc2)c2c([O-])ccc3[nH]ccc23)c1OC</smiles>

CCDC No. -1936470

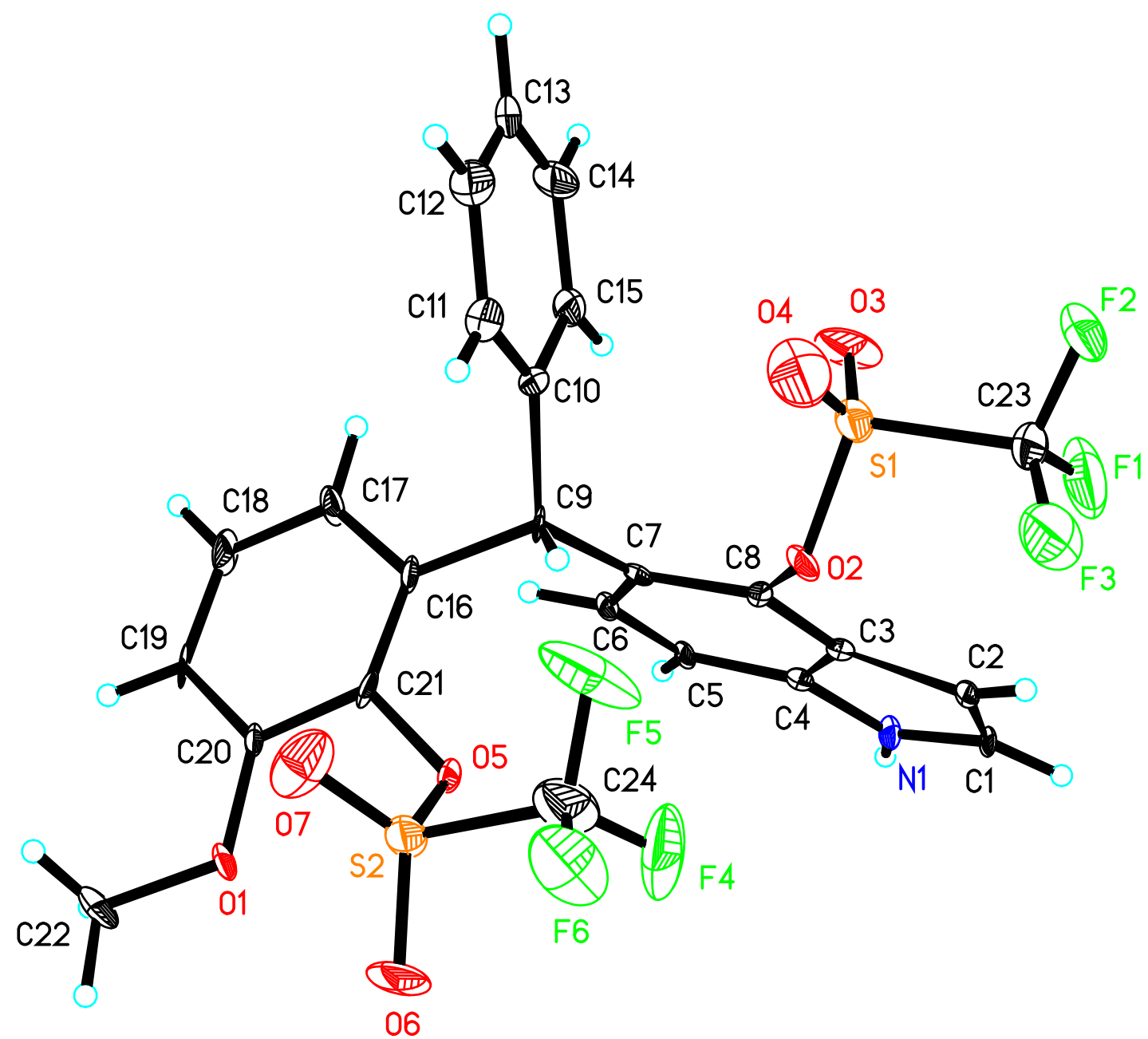

Figure S.1. X-ray structure of compound 4. An ethyl acetate solvent molecule is omitted for clarity. The thermal ellipsoids are drawn at the $30 \%$ probability level.

The single crystal of chiral compound 4, which was used for the determination of its absolute configuration via X-ray crystallography (see below), was obtained through slow evaporation of a solution in ethyl acetate and $n$-hexane at room temperature. The X-ray source used for the single crystal X-ray diffraction analysis of compound 4 was Mo $\mathrm{K} \alpha(\lambda=0.71073 \AA)$. CCDC 1936470 contains the structure and supplementary crystallographic data for the structure of compound 4 . These data can be obtained free of charge via www.ccdc.com.ac.uk/data_request/cif from the Cambridge. 
Table S.1. Crystal data and structure refinement for compound $\mathbf{4}$

\begin{tabular}{|c|c|c|}
\hline Identification code & \multicolumn{2}{|l|}{1936470} \\
\hline Empirical formula & \multicolumn{2}{|l|}{$\mathrm{C}_{28} \mathrm{H}_{25} \mathrm{~F}_{6} \mathrm{NO}_{9} \mathrm{~S}_{2}$} \\
\hline Formula weight & \multicolumn{2}{|l|}{697.61} \\
\hline Temperature & \multicolumn{2}{|l|}{ 193(2) K } \\
\hline Crystal system & \multicolumn{2}{|l|}{ Monoclinic } \\
\hline Space group & \multicolumn{2}{|l|}{ P 21} \\
\hline \multirow[t]{3}{*}{ Unit cell dimensions } & $\mathrm{a}=12.742(3) \AA$ & $\alpha=90^{\circ}$ \\
\hline & $\mathrm{b}=9.0469(19) \AA$ & $\beta=96.544(9)^{\circ}$. \\
\hline & $c=26.924(6) \AA$ & $\gamma=90^{\circ}$. \\
\hline Volume & \multicolumn{2}{|l|}{$3083.5(11) \AA^{3}$} \\
\hline $\mathrm{Z}$ & \multicolumn{2}{|l|}{4} \\
\hline Density (calculated) & \multicolumn{2}{|l|}{$1.503 \mathrm{Mg} / \mathrm{m}^{3}$} \\
\hline Absorption coefficient & \multicolumn{2}{|l|}{$0.262 \mathrm{~mm}^{-1}$} \\
\hline $\mathrm{F}(000)$ & \multicolumn{2}{|l|}{1432} \\
\hline Crystal size & \multicolumn{2}{|c|}{$0.200 \times 0.120 \times 0.080 \mathrm{~mm}^{3}$} \\
\hline Theta range for data collection & \multicolumn{2}{|l|}{2.085 to $23.905^{\circ}$. } \\
\hline Index ranges & \multicolumn{2}{|c|}{$-11<=\mathrm{h}<=14,-10<=\mathrm{k}<=10,-25<=\mathrm{l}<=30$} \\
\hline Reflections collected & \multicolumn{2}{|l|}{9815} \\
\hline Independent reflections & \multicolumn{2}{|c|}{$7860[\mathrm{R}(\mathrm{int})=0.0345]$} \\
\hline Completeness to theta $=25.242^{\circ}$ & \multicolumn{2}{|l|}{$83.7 \%$} \\
\hline Absorption correction & \multicolumn{2}{|c|}{ Semi-empirical from equivalents } \\
\hline Max. and min. transmission & \multicolumn{2}{|l|}{0.7456 and 0.5457} \\
\hline Refinement method & \multicolumn{2}{|c|}{ Full-matrix least-squares on $\mathrm{F}^{2}$} \\
\hline Data / restraints / parameters & \multicolumn{2}{|l|}{$7860 / 39 / 835$} \\
\hline Goodness-of-fit on $\mathrm{F}^{2}$ & \multicolumn{2}{|l|}{1.034} \\
\hline Final $R$ indices $[I>2 \operatorname{sigma}(I)]$ & \multicolumn{2}{|c|}{$\mathrm{R} 1=0.0878, \mathrm{wR} 2=0.2305$} \\
\hline $\mathrm{R}$ indices (all data) & \multicolumn{2}{|c|}{$\mathrm{R} 1=0.1251, \mathrm{wR} 2=0.2687$} \\
\hline Absolute structure parameter & \multicolumn{2}{|l|}{$0.03(6)$} \\
\hline Extinction coefficient & \multicolumn{2}{|l|}{$\mathrm{n} / \mathrm{a}$} \\
\hline Largest diff. peak and hole & \multicolumn{2}{|c|}{1.062 and -0.551 e. $\AA^{-3}$} \\
\hline
\end{tabular}

